\title{
Cherkis bow varieties and Coulomb branches of quiver gauge theories of affine type $A$
}

\author{
Hiraku Nakajima and Yuuya Takayama
}

\begin{abstract}
We show that Coulomb branches of quiver gauge theories of affine type $A$ are Cherkis bow varieties, which have been introduced as ADHM type description of moduli space of instantons on the Taub-NUT space equivariant under a cyclic group action.
\end{abstract}

\section{Introduction}

We have two purposes of this paper. The first is to initiate algebro-geometric study of Cherkis bow varieties $\mathcal{M}$ of affine type $A$, which have been introduced as ADHM type description of moduli spaces of $\mathrm{U}(n)$-instantons on the Taub-NUT space equivariant under a cyclic group $\mathbb{Z} / \ell \mathbb{Z}$-action [Che11] (see also [Che09, Che10]). Our interest lies in the Uhlenbeck partial compactification of instanton moduli spaces. They are algebraic varieties with hyper-Kähler structure on their regular loci, with an $\mathrm{SU}(2)$-action rotating three complex structures. They include quiver varieties of affine type $A$ introduced by the first-named author [Nak94b] as special cases, but more general. We introduce quiver description of arbitrary bow varieties (Theorem 2.1). See Figure 1. The original definition involves Nahm's equation, which is an ordinary differential equation, while quiver description involves matrices and algebraic equations. Hence the latter is more suitable for algebro-geometric questions. For example, quiver description enabels us to show that bow varieties are normal in many cases. The proof of the quiver description of a bow variety is a simple combination of known results [Don84, Hur89, Tak16]. See paragraphs after Theorem 2.1 for more detail. Nevertheless we think that it is important, as it is easier to handle. We believe that it has applications to other problems on bow varieties. It is also expected that bow varieties are related to representation theory of affine Lie algebras of type $A$, as for quiver varieties, but in a better way. We will study stratifications, semismall resolutions, torus fixed points, etc for bow varieties, as they have played important roles in relation between quiver varieties and representation theory.

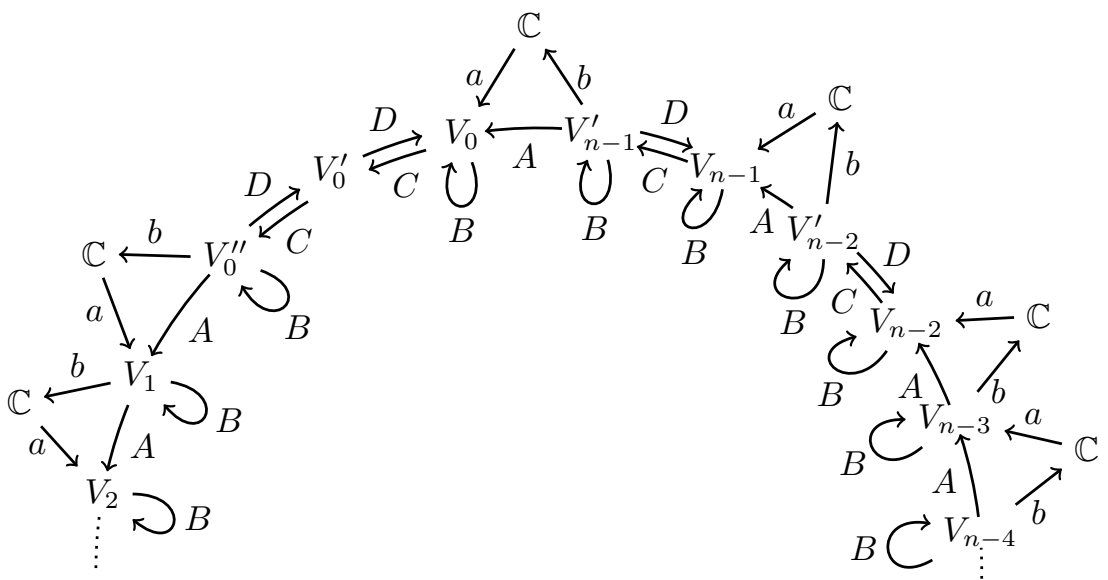

Figure 1: Quiver description of a bow variety 
The second purpose is to show that bow varieties also include Coulomb branches of framed quiver gauge theories of affine type $A$ again as special cases.

Let us recall what are Coulomb branches briefly. Let $G$ be a complex reductive group and $\mathbf{M}$ its symplectic representation. The Coulomb branch $\mathcal{M}_{C}$ of a $3 d \mathcal{N}=4$ SUSY gauge theory associated with $(G, \mathbf{M})$ is a hyper-Kähler manifold, and had been studied by physicists for many years. But its mathematical definition was unclear until the first named author proposed a rigorous approach in [Nak16]. The subsequent paper [BFN16a] written with Braverman and Finkelberg gives a mathematical definition of $\mathcal{M}_{C}$ as an affine algebraic variety, under the assumption that $\mathbf{M}$ is of form $\mathbf{N} \oplus \mathbf{N}^{*}$. The companion paper [BFN16b] by the same authors discusses $\mathcal{M}_{C}$ for a framed/unframed quiver gauge theory of type $A D E$. In particular, it was shown that $\mathcal{M}_{C}$ is isomorphic to a slice in the affine Grassmannian of the corresponding group or its generalization (generalized slice).

In this paper we consider the Coulomb branch of a framed quiver gauge theory of affine type $A_{n-1}(n \geq 1)$. We have two dimension vectors $\underline{\mathbf{v}}=\left(\mathbf{v}_{0}, \ldots, \mathbf{v}_{n-1}\right), \underline{\mathbf{w}}=\left(\mathbf{w}_{0}, \ldots, \mathbf{w}_{n-1}\right)$ and consider the gauge theory with the group $G=\prod \mathrm{GL}\left(\mathbf{v}_{i}\right)$ and the representation $\mathbf{N}=$ $\bigoplus \operatorname{Hom}\left(\mathbb{C}^{\mathbf{v}_{i}}, \mathbb{C}^{\mathbf{v}_{i+1}}\right) \oplus \bigoplus \operatorname{Hom}\left(\mathbb{C}^{\mathbf{w}_{i}}, \mathbb{C}^{\mathbf{v}_{i}}\right)$. Our goal is to identify $\mathcal{M}_{C}$ with Cherkis bow variety $\mathcal{M}$. Here $\ell$ is the level of the affine weight $\lambda:=\sum \mathbf{w}_{i} \Lambda_{i}$. Other discrete data (e.g., instanton number) are determined by $\mathbf{v}_{i}, \mathbf{w}_{i}$. A little more precisely, we assume $\operatorname{dim} V_{i}=\operatorname{dim} V_{i}^{\prime}=\operatorname{dim} V_{i}^{\prime \prime}=\cdots$ (the balanced condition), and the dimension of $W_{i}$ is encoded in the quiver as the number of vector spaces with primes between $V_{i}$ and $V_{i+1}$ in Figure 1. Hence $\ell$ is the number of arrows for $C$ (also for $D$ ), and $n$ is the number of arrows for $A$ (and $a, b)$.

This answer was known in the physics literature [HW97, dBHOO97, $\mathrm{dBHO}^{+}$97, CK98] for various cases. The general answer as a bow variety with the balanced condition was informed to us by Cherkis in a private communication. See [COS11] for a physical derivation. Therefore the main result of this paper could be regarded as a yet another confirmation of correctness of the proposal [Nak16].

Our strategy to identify the Coulomb branch $\mathcal{M}_{C}$ with a bow variety $\mathcal{M}$ is the same as in [BFN16b]: The Coulomb branch $\mathcal{M}_{C}$ has a morphism $\varpi: \mathcal{M}_{C} \rightarrow \mathbb{A} \mathbf{v}:=\prod \mathbb{A}^{\mathbf{v}_{i}} / \mathfrak{S}_{\mathbf{v}_{i}}$, where $\mathbf{v}_{i}=\operatorname{dim} V_{i}$. It satisfies the factorization property, i.e., $\mathcal{M}_{C}$ is isomorphic to a product of lower dimensional Coulomb branches, locally around $x$ if $\varpi(x)$ is a sum of disjoint configurations. We define the corresponding factorization map $\Psi: \mathcal{M} \rightarrow \mathbb{A} \underline{\mathbf{v}}$ for the bow variety, and prove the factorization property. We then define a birational isomorphism $\Xi^{\circ}: \mathcal{M}_{C} \underset{-\rightarrow}{\sim} \mathcal{M}$ over $\mathbb{A} \underline{\mathbf{v}}$ and show that it is biregular up to codimension 2. Then we use the criterion in [BFN16a, Th. 5.26] to conclude that $\Xi^{\circ}$ extends to the whole space.

The morphism $\varpi$ is an integrable system, i.e., components are Poisson commuting and general fibers are algebraic torus. For $n=1$, it is the cyclotomic, rational or trigonometric Calogero-Moser system depending on $\operatorname{dim} W$, but probably new one for $n>1$. We only use it to identify Coulomb branches with bow varieties in this paper, but it is certainly worthwhile to pursue its study.

Remark 1.1. In [Nak16], it was conjectured that $\mathcal{M}_{C}$ is a quiver variety of an affine type $A_{\ell-1}$ introduced in [Nak94b], under the assumption that $\mu:=\sum_{i} \mathbf{w}_{i} \Lambda_{i}-\mathbf{v}_{i} \alpha_{i}$ is dominant. Recall that a quiver variety of affine type $A_{\ell-1}$ is a moduli space of $\mathrm{U}(n)$-instantons on $\mathbb{R}^{4}$ equivariant under the cyclic group $\mathbb{Z} / \ell \mathbb{Z}$. The Taub-NUT space and $\mathbb{R}^{4}$ are isomorphic as holomorphic symplectic manifolds but have different hyper-Kähler metrics. We will also prove this conjecture from our main result, but the bow variety is regarded as a correct answer for $\mathcal{M}_{C}$ as

1. it covers also non-dominant cases,

2. the conjectural hyper-Kähler metric is correct,

3. several important properties of $\mathcal{M}_{C}$ can be checked for bow varieties rather naturally, but not for quiver varieties.

As for 1 , for example when $\ell=1$, the dominant condition is satisfied only when $\sum \mathbf{v}_{i} \alpha_{i}$ is a multiple of the primitive imaginary root. For 3 , the factorization map $\Psi$ is constructed as a bow variety, but its description in a quiver variety is not obvious. 
The first named author learned the subtle difference between quiver and bow varieties after reading [BDG15].

In order to apply [BFN16a, Th. 5.26], we need to check that $\mathcal{M}$ is normal and all the fibers of $\Psi$ have the same dimension. These conditions are delicate, and we use the quiver description in essential way.

The quiver is divided into two types of pieces, triangle involving $A, B, a, b$, and two-way involving $C, D$. When there are no two-way parts (corresponding to the case $\mathbf{w}=0$ ), the quiver is called the chainsaw quiver considered in [FR14]. The normality and the calculation of dimension of fibers of $\Psi$ for triangles were already established in [FR14]. In fact, the identification of the Coulomb branch in this case was already given in [BFN16b] by using [FR14]. The remaining two-way parts are quiver varieties of type $A$ in the sense of [Nak94b, Nak98]. The proof of the normality and calculation of dimension were given in [CB01, CB03a] (in fact, in much more general situations). Our proof of the normality of bow varieties are basically consequences of these facts.

Recall that the balanced condition is $\operatorname{dim} V_{i}=\operatorname{dim} V_{i}^{\prime}=\cdots$. When we assume the cobalanced ${ }^{1}$ condition that dimensions of vector spaces connected by an arrow $A$ in Figure 1 are the same, the bow variety is isomorphic to a quiver variety $\mathfrak{M}$ introduced in [Nak94b] of an affine type $A_{n-1}$. This is what we have mentioned at the beginning.

There is an isomorphism between two bow varieties where adjacent triangle and two-way parts are swapped where dimension of the middle vector space is changed appropriately. See Proposition 7.1. For example, consider two parts for $V_{0}^{\prime}, V_{0}^{\prime \prime}, V_{1}$ in Figure 1. We can exchange triangle and two-way parts. Vector spaces $V_{0}^{\prime}, V_{1}$ are unchanged, and the dimension of the new middle vector space becomes $\operatorname{dim} V_{1}+\operatorname{dim} V_{0}^{\prime}+1-\operatorname{dim} V_{0}^{\prime \prime}$. It appeared as Hanany-Witten transition in [HW97]. This is a powerful tool, which does not have analog in the context of quiver varieties. As applications, we prove

- Under the dominance assumption mentioned in Remark 1.1, a bow variety satisfying the balanced condition is isomorphic to one satisfying the cobalanced condition by successive application of Hanany-Witten transitions.

- We give a new proof of [Nak94b, Maf05] saying a quiver variety of type $A$ is isomorphic to the intersection of Slodowy slice and a nilpotent orbit or its Springer resolution.

- We determine the symplectic leaves of the Coulomb branch $\mathcal{M}_{C}$. (This is new even for finite type $A$.)

We have the duality of bow varieties exchanging triangle and two-way parts without changing dimensions of vector spaces $V$ 's. It does not induces an isomorphism of bow varieties, but it originates from the $\mathrm{SL}(2, \mathbb{Z})$-duality in the superstring theory, and closely related to the $3 d$ mirror symmetry. (See [HW97].) Therefore we expect two bow varieties exchanged by the duality have some deep relations. For example, the balanced and cobalanced conditions are exchanged under the duality. Thus a quiver variety $\mathfrak{M}$ and a Coulomb branch $\mathcal{M}_{C}$ are exchanged by this process. When the dominance condition is satisfied, it gives an exchange of two quiver varieties. Chasing changes of dimensions under successive applications of Hanany-Witten transitions, we will find that pairs of weights $(\lambda, \mu)$ are replaced by their 'transpose' as generalized Young diagrams, as expected from the relation between the level-rank duality and quiver varieties. (See [Nak09, §A].)

The quiver description of a bow variety can be understood as hamiltonian reduction of a finite dimensional symplectic manifold. One can consider the corresponding quantum hamiltonian reduction following [FR14] for the case $\mathbf{w}=0$. We conjecture that it is isomorphic to the quantized Coulomb branch when the balanced condition is satisfied.

Remark 1.2. As we have remarked above, bow varieties are moduli spaces of $\mathrm{U}(n)$-instantons on the Taub-NUT space equivariant under the $\mathbb{Z} / \ell \mathbb{Z}$-action, though mathematically rigorous proof of the completeness is still lacking as far as the authors know. Let us give other moduli theoretic description, but with more algebro-geometric flavor, known for special classes. However different

${ }^{1}$ This terminology is due to Sergey Cherkis. 
stability conditions were imposed (except in [Tak16]), thus the moduli spaces are not exactly the same as bow varieties.

- Chainsaw quiver varieties [FR14] are moduli spaces of framed parabolic sheaves on $\mathbb{P}^{1} \times \mathbb{P}^{1}$. Here parabolic structures are put on $\{0\} \times \mathbb{P}^{1}$, while framing is put on $\{\infty\} \times \mathbb{P}^{1} \cup \mathbb{P}^{1} \times\{\infty\}$. With the stability condition (C-S1),(C-S2) below, they are open loci of framed parabolic locally free sheaves [Tak16].

- If the bow diagram has both triangle and two-way parts only once, the varieties describe moduli spaces of framed rank 1 stable perverse coherent sheaves on the blowup $\widehat{\mathbb{P}}^{2}$ of $\mathbb{P}^{2}$ at the origin [NY11]. Here the framing is put on the line at infinity. This quiver description was originated in [Kin89].

- If there is only one two-way part, the quiver is called the dented chainsaw quiver [BF13]. The varieties describe moduli spaces of framed parabolic sheaves on $\widehat{\mathbb{P}}^{2}$.

- The rift quiver introduced in [BF13] is a variant of our quiver. The varieties describe moduli spaces of $\mathbb{Z} / \ell \mathbb{Z}$-equivariant framed parabolic sheaves on $\widehat{\mathbb{P}}^{2}$, where $\mathbb{Z} / \ell \mathbb{Z}$ acts on $\widehat{\mathbb{P}}^{2}$ by $\left(\left[z_{0}: z_{1}: z_{2}\right],[z: w]\right) \mapsto\left(\left[z_{0}: \zeta z_{1}: z_{2}\right],\left[z: \zeta^{-1} w\right]\right)$.

Here $\widehat{\mathbb{P}}^{2}$ is $\left\{\left(\left[z_{0}: z_{1}: z_{2}\right],[z: w]\right) \in \mathbb{P}^{2} \times \mathbb{P}^{1} \mid z_{1} w=z_{2} z\right\}$, the line at infinity is $z_{0}=0$, and paraoblic structures are defined on the line $z=0$. Based on these examples, we expect that bow varieties are isomorphic to Uhlenbeck-type partial compactification of moduli spaces of $\mathbb{Z} / \ell \mathbb{Z}$-equivariant framed parabolic locally free sheaves on $\widehat{\mathbb{P}}^{2}$. The Taub-NUT space is embedded into $\widehat{\mathbb{P}}^{2}$ as an open subvariety $z_{0} \neq 0, z \neq 0$, which is isomorphic to $\mathbb{C}^{2}$.

The paper is organized as follows. In $\S 2$ we review the definition of Cherkis bow varieties. We also explain the quiver description. In $\S 3$ we study properties of triangle and two-way parts of bow varieties. In $\S 4$ we study stratification of bow varieties and semismall morphisms from a bow variety to another with different continuous parameters (stability conditions). In $\S 5$ we study symplectic structure on a bow variety. In particular, we show that it is given by a reduction of a finite dimensional Poisson manifold. In $\S 6$ we show that the Coulomb branch $\mathcal{M}_{C}$ is isomorphic to the bow variety $\mathcal{M}$. In $\S 7$ we study Hanany-Witten transition as an isomorphism between two bow varieties. We derive several applications mentioned above.

\section{Acknowledgments}

It is clear that the authors owe a lot to Sergey Cherkis. They are grateful to him for his explanation on bow varieties over years. The first-named author also thanks A. Braverman and M. Finkelberg for useful discussions. We further thank M. Finkelberg for pointing out mistakes on flavor symmetry in an earlier version.

The research of H.N. is supported by JSPS Kakenhi Grant Numbers 23224002, 23340005, $24224001,25220701$.

\section{Bow varieties}

Let us review the definition of a bow variety of affine type $A_{\ell-1}(\ell \geq 1)$ [Che11] and give its quiver description. We will regard a bow variety as an affine algebraic variety with holomorphic symplectic form on its regular locus. Then its quiver description does not loose any information. Therefore readers who are not interested in the original definition can safely skip the first part $\S 2.1$, and regard the quiver description in the second part $\S 2.2$ as the definition. We will use the original definition only in $\S 6.9 .3$.

Let us first explain the data which are common in both original definition and the quiver description. It is a bow diagram. 
We take an affine Dynkin quiver of type $A_{\ell-1}$ with the anticlockwise orientation. We understand $A_{0}$ is the Jordan quiver. We replace vertices of quiver by wavy lines $\sim$ possibly with $\times$ on them. A wavy line without $\times$ is allowed. Let $\mathcal{I}$ be the set of wavy lines. We call $\times$ a $x$-point. Let $\Lambda$ denote the set of $x$-points. (It was called a $\lambda$-point in [Che11], but we keep $\lambda$ for a weight.) We orient wavy lines in the anticlockwise direction. Wavy lines are denoted by $\sigma, \sigma^{\prime}, \ldots$, etc and we identify them with $0,1, \ldots, \ell-1$, or elements in $\mathbb{Z} / \ell \mathbb{Z}$. For a later purpose, we index them in the clockwise order. We also index the arrow by $0,1, \ldots, \ell-1$, where $i$ th arrow is between the end of $i$ th wavy line and the start of the $(i+1)$ th wavy line.

Let $n$ be the number of $x$-points. We index them as $0,1, \ldots, n-1$ in the anticlockwise order. The number $n$ will play the role of level of affine weights. When bow varieties will be identified with Coulomb branches of gauge theories, level and rank are exchanged.

A segment of a wavy line is a part which is cut out by $x$-points. An $x$-point is denoted by $x$, and a segment by $\zeta$. Let $\mathrm{o}(\zeta), \mathrm{i}(\zeta)$ denote the starting and ending points of $\zeta$ respectively, i.e., $\mathrm{o}(\zeta) \sim{ }^{\zeta} \sim \mathrm{i}(\zeta)$. Here $\mathrm{o}(\zeta), \mathrm{i}(\zeta)$ are either end points of wavy lines or $x$-points. An oriented edge is denoted by $h$, and its starting and ending points are denoted also by o $(h), \mathrm{i}(h)$, i.e., o $(h) \stackrel{h}{\longrightarrow} \mathrm{i}(h)$. Here $\mathrm{o}(h), \mathrm{i}(h)$ are end points of wavy lines. There should be no fear of confusion for using the same notation for end points of segments and edges.

We assign a nonnegative integer $R(\zeta)$ to each segment $\zeta$ of wavy lines. These are discrete data for a bow variety. We put $R(\zeta)$ next to $\zeta$. Figure $2(\mathrm{a})$ is the bow diagram for the example in Figure 1 with $R(\zeta)=\operatorname{dim} V_{i}, \operatorname{dim} V_{i}^{\prime}=\mathbf{v}_{i}, \mathbf{v}_{i}^{\prime}$ etc. A simplified version of the diagram suffices for most of our purposes: we replace an arrow by $\bigcirc$ and remove waves from lines. See Figure 2(b).

We understand $\mathbf{v}_{i}$ as a part of the bow diagram. Considering $\left(\mathbf{v}_{0}, \mathbf{v}_{0}^{\prime}, \ldots\right)$ as a vector, we call it the dimension vector of the bow diagram.

For our later purpose, it is better to index $x$-points as $0,1, \ldots, n-1$. We take the $j$ th $x$-point and index vector spaces on segments as $V_{j}, V_{j}^{\prime}, \ldots$, anticlockwise until we meet the next $x$-point. See Figure 1.

The bow diagram originates from a brane configuration in type IIB string theory [HW97]. Oriented edges $\rightarrow$ correspond to NS 5-branes, while $x$-points correspond to Dirichlet 5-branes. Integers $R(\zeta)$ represent the number of Dirichlet 3-branes running between either type of branes. The configuration is drawn as Figure 3, where radial full lines are Dirichlet 5-branes, radial dotted lines are NS 5-branes, and circular lines are Dirichlet 3-branes. See [dBHOO97] for affine cases. Note that the lines for NS and Dirichlet 5-branes are opposite of [HW97, dBHOO97], as bow varieties are Coulomb branches of dual theories by [COS11]. We will not (and could not) use branes, hence this difference will not be important later.

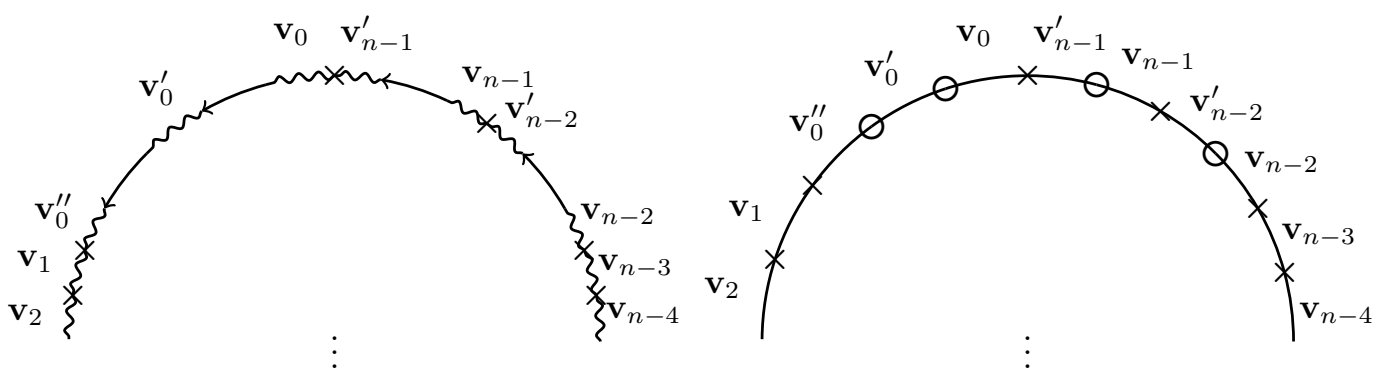

Figure 2: (a) A bow diagram of affine type $A_{\ell-1}$ and (b) its simplified version

We choose a parameter $\nu_{\sigma}=\left(\nu_{\sigma}^{1}, \nu_{\sigma}^{2}, \nu_{\sigma}^{3}\right) \in \sqrt{-1} \mathbb{R}^{3}$ for each wavy line (or arrow) $\sigma$. In the quiver description, we consider $\mathbb{R}^{3}=\mathbb{R} \times \mathbb{C}$ and denote it by $\nu_{\sigma}=\left(\nu_{\sigma}^{\mathbb{R}}, \nu_{\sigma}^{\mathbb{C}}\right)$ with $\nu_{\sigma}^{1}=\sqrt{-1} \nu_{\sigma}^{\mathbb{R}}$, $\nu_{\sigma}^{2}+\sqrt{-1} \nu_{\sigma}^{3}=\nu_{\sigma}^{\mathbb{C}}$. When we drop $\sigma$, it means a vector, e.g., $\nu \in\left(\sqrt{-1} \mathbb{R}^{3}\right)^{\mathcal{I}}$. When we use $\nu_{\sigma}^{\mathbb{R}}$ in the quiver description, we assume $\nu_{\sigma}^{\mathbb{R}} \in \mathbb{Q}$. 


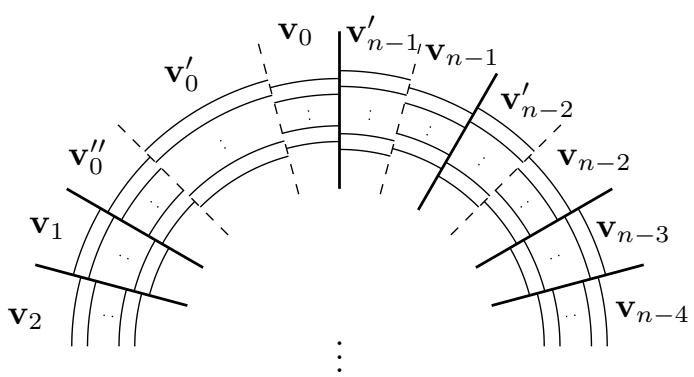

Figure 3: Brane configuration

\subsection{Original definition}

We further add the following data.

Let $\sigma$ be a wavy line. We assign an interval $\left[x_{\sigma, L}, x_{\sigma, R}\right]$. We assume those intervals are mutually disjoint. Let $r_{\sigma}$ be the number of $\times$ on $\sigma$. We choose $x_{\sigma, L}<x_{\sigma}^{1}<\cdots<x_{\sigma}^{r_{\sigma}}<x_{\sigma, R}$ corresponding to $x$-points in $\sigma$ in the anticlockwise order. We set $x_{\sigma}^{0}=x_{\sigma, L}, x_{\sigma}^{r_{\sigma}+1}=x_{\sigma, R}$. We now regard $\Lambda \subset \mathbb{R}$. We identify a segment $\zeta$ with the closed interval $[\mathrm{o}(\zeta), \mathrm{i}(\zeta)]$ in $\mathbb{R}$.

We assign one dimension complex vector space $\mathbb{C}_{x}$ with a hermitian inner product for each $x \in \Lambda$. When there is no fear of confusion, we simply denote it by $\mathbb{C}$, e.g., as in Figure 1 . In addition, we choose a hermitian vector bundle $E_{\zeta}$ of $\operatorname{rank} R(\zeta)$ for each segment $\zeta$.

Those data satisfy the matching condition at $x$-points. Let us suppose two segments $\zeta^{-}$, $\zeta^{+}$meet at $x$. We suppose $\mathrm{i}\left(\zeta^{-}\right)=x=\mathrm{o}\left(\zeta^{+}\right)$, i.e., ${\stackrel{\zeta^{-} x}{\zeta^{+}}}^{+}$. Let us consider the difference $\Delta R(x):=\left|R\left(\zeta^{-}\right)-R\left(\zeta^{+}\right)\right|$of ranks of two vector bundles defined over $\zeta, \zeta^{\prime}$. We suppose the fiber of the smaller (or equal to) rank vector bundle is a subspace of the fiber of the bigger (or equal to) rank vector bundle, i.e.,

$$
\begin{aligned}
& \left.\left.E_{\zeta^{-}}\right|_{x} \subset E_{\zeta^{+}}\right|_{x} \text { if } R\left(\zeta^{-}\right) \leq R\left(\zeta^{+}\right), \\
& \left.\left.E_{\zeta^{-}}\right|_{x} \supset E_{\zeta^{+}}\right|_{x} \text { if } R\left(\zeta^{-}\right) \geq R\left(\zeta^{+}\right) .
\end{aligned}
$$

Furthermore we endow a structure of an irreducible $\mathfrak{s u}(2)$ representation on the orthogonal complement $\left(\left.E_{\zeta^{-}}\right|_{x}\right)^{\perp}$ or $\left(\left.E_{\zeta^{+}}\right|_{x}\right)^{\perp}$. Its dimension is $\Delta R(x)$. We thus have three endomorphisms $\rho_{1}, \rho_{2}, \rho_{3}$ on the orthogonal complement satisfying $\rho_{1}=\left[\rho_{2}, \rho_{3}\right]$ and its cyclic permutation. We have $U(1)$-choices of identifications with the $\mathfrak{s u}(2)$ representation, which we consider as parts of data. (In the quiver description below, we break symmetries between 1,2,3 and take a triangular decomposition $\mathfrak{n} \oplus \mathfrak{h} \oplus \mathfrak{n}^{-}$of $\mathfrak{s l}(2)=\mathfrak{s u}(2) \otimes \mathbb{C}$. Then the identification will be identified with an isomorphism from the highest weight space to $\mathbb{C}_{x}$.) When there is no fear of the confusion, we drop the subscript $\zeta$ from $E_{\zeta}$. For example, we do not need to specify $\zeta$ for a fiber of $E_{\zeta}$ at a point $\notin \Lambda$, or a point $x \in \Lambda$ with $\Delta R(x)=0$.

Now a bow solution consists of the following data:

1. A hermitian connection $\nabla$ and skew hermitian endomorphisms $T_{1}, T_{2}, T_{3}$ on the vector bundle $E_{\zeta}$ over the open segment $(\mathrm{o}(\zeta), \mathrm{i}(\zeta))$ satisfying Nahm's equation

$$
\frac{\nabla}{d s} T_{1}+\left[T_{2}, T_{3}\right]=0, \quad \text { etc. }
$$

2. A pair of linear maps

$$
B^{L R}:\left.\left.E\right|_{\mathrm{o}(h)} \rightarrow E\right|_{\mathrm{i}(h)}, \quad B^{R L}:\left.\left.E\right|_{\mathrm{i}(h)} \rightarrow E\right|_{\mathrm{o}(h)},
$$

corresponding to an oriented arrow $h$ from o $(h)$ to $\mathrm{i}(h)$. Note $B^{R L}$ goes to the opposite direction. Note also that $\mathrm{o}(h)=x_{\sigma, R}, \mathrm{i}(h)=x_{\sigma+1, L}$ for a wavy line $\sigma$. Therefore they are not $x$-points, as $\stackrel{\mathrm{O}(h) \mathrm{i}(h)}{\longrightarrow}$. 
3. A pair of linear maps

$$
\begin{aligned}
I: \mathbb{C}_{x} & \left.\rightarrow E\right|_{x}, \\
J:\left.E\right|_{x} & \rightarrow \mathbb{C}_{x}
\end{aligned}
$$

for a point $x \in \Lambda$ such that $\Delta R(x)=0$.

We require that $\left(\nabla, T_{1}, T_{2}, T_{3}, B^{L R}, B^{R L}, I, J\right)$ satisfy the following conditions:

(a) $\nabla, T_{1}, T_{2}, T_{3}$ extend smoothly at $x_{\sigma, L}, x_{\sigma, R}$. Moreover they together with $B^{L R}, B^{R L}$ satisfy the hyper-Kähler moment map equation

$$
\begin{aligned}
-\frac{\sqrt{-1}}{2}\left\{B^{L R}\left(B^{L R}\right)^{\dagger}-\left(B^{R L}\right)^{\dagger} B^{R L}\right\}+T_{1}\left(x_{\sigma, L}\right) & =\nu_{\sigma}^{1} \mathrm{id}, \\
B^{L R} B^{R L}+\left(T_{2}+\sqrt{-1} T_{3}\right)\left(x_{\sigma, L}\right) & =\left(\nu_{\sigma}^{2}+\sqrt{-1} \nu_{\sigma}^{3}\right) \mathrm{id}
\end{aligned}
$$

at $x_{\sigma, L}$ and

$$
\begin{aligned}
-\frac{\sqrt{-1}}{2}\left\{B^{R L}\left(B^{R L}\right)^{\dagger}-\left(B^{L R}\right)^{\dagger} B^{L R}\right\}-T_{1}\left(x_{\sigma, R}\right) & =0 \\
-B^{R L} B^{L R}-\left(T_{2}+\sqrt{-1} T_{3}\right)\left(x_{\sigma, R}\right) & =0
\end{aligned}
$$

at $x_{\sigma, R}$.

(b) Let $x \in \Lambda$. Let $\zeta^{ \pm}$as before. We suppose $R\left(\zeta^{-}\right)<R\left(\zeta^{+}\right)$. (The condition for $R\left(\zeta^{-}\right)>$ $R\left(\zeta^{+}\right)$is the same after exchanging $E_{\zeta^{-}}$and $\left.E_{\zeta^{+}}\right)$We have $\nabla, T_{1}, T_{2}, T_{3}$ defined on $\zeta^{-}$, $\zeta^{+}$. Let us denote the former with - , and the latter with + . We require $\nabla^{ \pm}, T_{1}^{-}, T_{2}^{-}, T_{3}^{-}$ extend smoothly at $x$. On the other hand, we assume $T_{j}^{+}(j=1,2,3)$ behaves as

$$
T_{j}^{+}(s)=\left(\begin{array}{cc}
T_{j}^{-}(s)+O(s-x) & O\left((s-x)^{\frac{\Delta R(x)-1}{2}}\right. \\
O\left((s-x)^{\frac{\Delta R(x)-1}{2}}\right. & \frac{\rho_{j}}{s-x}+O(1)
\end{array}\right) .
$$

(c) Consider a point $x$ with $\Delta R(x)=0$. Let us put \pm on connections and endomorphisms as above. We require that $\nabla^{ \pm}, T_{1}^{ \pm}, T_{2}^{ \pm}, T_{3}^{ \pm}$are smooth at $x$, and they satisfy the hyper-Kähler moment map equation with $I, J$ :

$$
\begin{array}{r}
-\frac{\sqrt{-1}}{2}\left\{I I^{\dagger}-J^{\dagger} J\right)-T_{1}^{-}(x)+T_{1}^{+}(x)=0, \\
I J-\left(T_{2}^{-}+\sqrt{-1} T_{3}^{-}\right)(x)+\left(T_{2}^{+}+\sqrt{-1} T_{3}^{+}\right)(x)=0 .
\end{array}
$$

There is a natural gauge equivalence on these solutions: A gauge transformation $u$ consists of a unitary gauge transformation $u_{\zeta}$ of $E_{\zeta}$, which is smooth on $[\mathrm{o}(\zeta), \mathrm{i}(\zeta)]$ for each segment $\zeta$.

Moreover for ${\stackrel{\zeta}{\zeta} x \zeta^{+}}^{+}$, we assume $u_{\zeta^{-}}(x)=u_{\zeta^{+}}(x)$ when $\Delta R(x)=0$,

$$
u_{\zeta^{+}}(x)=\left(\begin{array}{cc}
u_{\zeta^{-}}(x) & 0 \\
0 & \operatorname{id}_{\left(E_{\zeta^{-} \mid x^{\prime}}\right)^{\perp}}
\end{array}\right)
$$

when $R\left(\zeta^{-}\right)<R\left(\zeta^{+}\right)$, and similarly when $R\left(\zeta^{-}\right)>R\left(\zeta^{+}\right)$. The action is given by

$$
\left(u_{\zeta} \circ \nabla \circ u_{\zeta}^{-1}, u_{\zeta} T_{1} u_{\zeta}^{-1}, u_{\zeta} T_{2} u_{\zeta}^{-1}, u_{\zeta} T_{3} u_{\zeta}^{-1}\right)
$$

for $\left(\nabla, T_{1}, T_{2}, T_{3}\right)$ defined on $E_{\zeta}$. Also by

$$
\left(u(\mathrm{i}(h)) B^{L R} u(\mathrm{o}(h))^{-1}, u(\mathrm{o}(h)) B^{R L} u(\mathrm{i}(h))^{-1}\right)
$$


for $B^{L R}, B^{R L}$ corresponding to $h$, where $u(\mathrm{i}(h)), u(\mathrm{o}(h))$ are evaluation of appropriate $u_{\zeta}$ at points $\mathrm{i}(h), \mathrm{o}(h)$ respectively. And by

$$
\left(u(x) I, J u(x)^{-1}\right)
$$

for $x \in \Lambda$ such that $\Delta R(x)=0$. Since $u_{\zeta^{-}}(x)=u_{\zeta^{+}}(x)$, we do not need to specify a segment for $u(x)$.

Let $\mathcal{M}_{\text {bow }}$ be the space of gauge equivalence classes of bow solutions. Let $\mathcal{M}_{\mathrm{bow}}^{\mathrm{reg}}$ be the subset consisting of classes of solutions which have trivial stabilizer. One introduces an appropriate Sobolev space to give a topology on $\mathcal{M}_{\text {bow }}$ such that $\mathcal{M}_{\text {bow }}^{\text {reg }}$ is an open subset with a hyper-Kähler structure, as an infinite dimensional hyper-Kähler quotient. See e.g., [Tak15]. The dimension of $\mathcal{M}_{\text {bow }}^{\text {reg }}$ can be computed, but we omit the formula as we will calculate it in the quiver description in Proposition 2.13.

\subsection{A quiver description of a bow variety}

We assign one dimensional complex vector space $\mathbb{C}_{x}$ for each $x \in \Lambda$ as before. We do not put hermitian inner products. We often omit $x$ as mentioned above.

In addition, we assign a vector space $V_{\zeta}$ for each segment $\zeta$ of a wavy line with $\operatorname{dim} V_{\zeta}=R(\zeta)$. For a quiver description, data consist of the following:

1. A linear endomorphism $B: V_{\zeta} \rightarrow V_{\zeta}$ for each segment $\zeta$. When we want to emphasize $\zeta$, we denote it by $B_{\zeta}$.

2. Let $x \in \Lambda$. Let $\zeta^{-}, \zeta^{+}$be the adjacent segments so that ${\stackrel{\zeta}{\sim} x \zeta^{+}}^{+}$is the anticlockwise orientation. We assign triple of linear maps

$$
\begin{gathered}
A: V_{\zeta^{-}} \rightarrow V_{\zeta^{+}}, \\
a: \mathbb{C}_{x} \rightarrow V_{\zeta^{+}}, \quad b: V_{\zeta^{-}} \rightarrow \mathbb{C}_{x} .
\end{gathered}
$$

When we want to emphasize $x$, we denote them by $A_{x}, a_{x}, b_{x}$.

3. A pair of linear maps

$$
C: V_{\mathrm{o}(h)} \rightarrow V_{\mathrm{i}(h)}, \quad D: V_{\mathrm{i}(h)} \rightarrow V_{\mathrm{o}(h)},
$$

for each arrow $h$. Here we regard $\mathrm{o}(h), \mathrm{i}(h)$ as segments containing the points and use the notation $V_{\mathrm{o}(h)}, V_{\mathrm{i}(h)}$ for brevity.

When we want to emphasize $h$, we denote them by $C_{h}, D_{h}$.

We require the following conditions:

(a) Let $x, \zeta^{ \pm}$as in 2 above. As a linear map $V_{\zeta^{-}} \rightarrow V_{\zeta^{+}}$we have

$$
B_{\zeta^{+}} A-A B_{\zeta^{-}}+a b=0 .
$$

They satisfy the two conditions (S1),(S2):

(S1) There is no nonzero subspace $0 \neq S \subset V_{\zeta^{-}}$with $B_{\zeta^{-}}(S) \subset S, A(S)=0=b(S)$.

(S2) There is no proper subspace $T \subsetneq V_{\zeta^{+}}$with $B_{\zeta^{+}}(T) \subset T, \operatorname{Im} A+\operatorname{Im} a \subset T$.

(b) As endomorphisms on $V_{\mathrm{i}(h)}$ and $V_{\mathrm{o}(h)}$, we have

$$
C D+B_{\mathrm{i}(h)}=\nu_{\mathrm{i}(h)}^{\mathbb{C}}, \quad-D C-B_{\mathrm{o}(h)}=0,
$$

respectively. We identify $\mathrm{i}(h)$ with the wavy line containing it, and consider the corresponding parameters $\nu$.

By these equations, we can safely remove $B_{\zeta}$ when $\zeta$ is connected with an arrow $h$. We will usually remove $B_{\zeta}$ when $\zeta$ is connected with arrows in both sides, i.e., when $\zeta$ is a wavy line without $x$-points. But we keep $B_{\zeta}$ otherwise. See $V_{0}^{\prime}$ at Figure 1. 
We introduce the following space of linear maps:

$$
\begin{gathered}
\mathbb{M}:=\bigoplus_{x} \operatorname{End}\left(V_{\zeta_{x}^{-}}\right) \oplus \operatorname{End}\left(V_{\zeta_{x}^{+}}\right) \oplus \operatorname{Hom}\left(V_{\zeta_{x}^{-}}, V_{\zeta_{x}^{+}}\right) \oplus \operatorname{Hom}\left(\mathbb{C}_{x}, V_{\zeta_{x}^{+}}\right) \oplus \operatorname{Hom}\left(V_{\zeta_{x}^{-}}, \mathbb{C}_{x}\right) \\
\oplus \bigoplus_{h} \operatorname{Hom}\left(V_{\mathrm{o}(h)}, V_{\mathrm{i}(h)}\right) \oplus \operatorname{Hom}\left(V_{\mathrm{i}(h)}, V_{\mathrm{o}(h)}\right) .
\end{gathered}
$$

We denote segments in 2 by $\zeta_{x}^{ \pm}$and elements in the component $\operatorname{End}\left(V_{\zeta_{x}^{-}}\right)$, $\operatorname{End}\left(V_{\zeta_{x}^{+}}\right)$by $B_{\zeta_{x}^{-}}$and $B_{\zeta_{x}^{+}}$as they also depend on $x$. Note that $\zeta_{x}^{+}=\zeta_{x^{\prime}}^{-}$if $\sim x \sim x^{\prime}$. Hence we may have two $B$ assigned for a single segment $\zeta$. But we will kill one of them by the relation soon.

We define

$$
\begin{aligned}
& \mu=\left(\mu_{1}, \mu_{2}\right): \mathbb{M} \rightarrow \mathbb{N}:=\bigoplus_{x} \operatorname{Hom}\left(V_{\zeta_{x}^{-}}, V_{\zeta_{x}^{+}}\right) \oplus \bigoplus_{\zeta} \operatorname{End}\left(V_{\zeta}\right) \\
& \mu_{1}:\left(A_{x}, B_{\zeta_{x}^{+}}, B_{\zeta_{x}^{-}}, a_{x}, b_{x}, C_{h}, D_{h}\right) \mapsto B_{\zeta_{x}^{+}} A_{x}-A_{x} B_{\zeta_{x}^{-}}+a_{x} b_{x},
\end{aligned}
$$

and the second component $\mu_{2}$ is depending on types of two ends of the wavy line $\zeta$ :

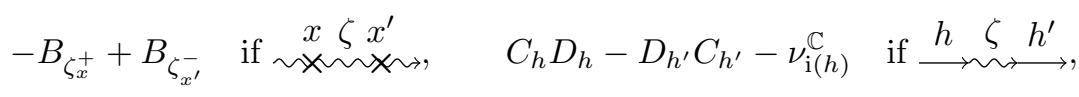

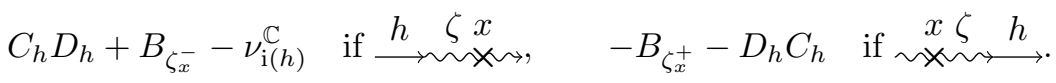

We will consider $\mu^{-1}(0)$, and the original $B_{\zeta}$ is given by $B_{\zeta_{x}^{+}}=B_{\zeta_{x}^{-}}$. We will use this convention unless we need to distinguish $B_{\zeta_{x}^{+}}$and $B_{\zeta_{x}^{-}}$.

We have a natural group action of $\mathcal{G}:=\prod \mathrm{GL}\left(V_{\zeta}\right)$ by conjugation, that is given by

$$
\left(g_{\zeta_{x}^{ \pm}} B_{\zeta_{x}^{ \pm}} g_{\zeta_{x}^{ \pm}}^{-1}, g_{\zeta_{x}^{+}} A_{x} g_{\zeta_{x}^{-}}^{-1}, g_{\zeta_{x}^{+}} a_{x}, b_{x} g_{\zeta_{x}^{-}}^{-1}, g_{\mathrm{i}(h)} C g_{\mathrm{o}(h)}^{-1}, g_{\mathrm{o}(h)} D g_{\mathrm{i}(h)}^{-1}\right) .
$$

(c) We impose an additional (semi)stability condition with respect to the group action of $\mathcal{G}$ as follows.

Let $\widetilde{\mathcal{M}}$ denote the variety consisting of $(A, B, a, b, C, D) \in \mu^{-1}(0)$ satisfying $(\mathrm{S} 1),(\mathrm{S} 2)$. As we will review Proposition 3.2 below, it is an affine algebraic variety. We assume $\nu_{\sigma}^{\mathbb{R}}$ is an integer and consider a character

$$
\chi: \mathcal{G} \rightarrow \mathbb{C}^{\times} ;\left(g_{\zeta}\right) \mapsto \prod\left(\operatorname{det} g_{\zeta}\right)^{-\nu_{\sigma}^{\mathbb{R}}}
$$

where $\zeta$ is the last segment in a wavy line $\sigma, \sim$, and the product is only over the last segments. Let $\mathcal{G}$ act on $\widetilde{\mathcal{M}} \times \mathbb{C}$ by $g \cdot(m, z)=\left(g m, \chi(g)^{-1} z\right)$ for $g \in \mathcal{G}, m \in \widetilde{\mathcal{M}}, z \in \mathbb{C}$. We say $m \in \widetilde{\mathcal{M}}$ is $\nu^{\mathbb{R}}$-semistable if the closure of the orbit $\mathcal{G}(m, z)$ does not intersect with $\widetilde{\mathcal{M}} \times\{0\}$ for $z \neq 0$. We say $m$ is $\nu^{\mathbb{R}}$-stable if the orbit $\mathcal{G}(m, z)$ is closed and the stabilizer of $(m, z)$ is finite for $z \neq 0$.

It is clear from Proposition 2.8 below that the (semi)stability condition is not effected under the change $\nu^{\mathbb{R}} \rightarrow c \nu^{\mathbb{R}}$ for $c \in \mathbb{Z}_{>0}$. Hence the (semi)stability condition is well-defined for rational $\nu^{\mathbb{R}}$.

Let $\widetilde{\mathcal{M}}$ ss $\left(\right.$ resp. $\widetilde{\mathcal{M}}$ ) denote the set of $\nu^{\mathbb{R}}$-semistable (resp. $\nu^{\mathbb{R}}$-stable) points.

We introduce the $S$-equivalence relation $\approx$ on the $\widetilde{\mathcal{M}}^{\text {ss }}$ by defining $m \sim m^{\prime}$ if and only if orbit closures $\overline{\mathcal{G} m}$ and $\overline{\mathcal{G} m}$ intersect in the $\widetilde{\mathcal{M}}^{\text {ss }}$. Let $\mathcal{M}_{\text {quiver }}$ denote $\widetilde{\mathcal{M}}^{\text {ss }} / \sim$. It is well-known that $\widetilde{\mathcal{M}}^{\text {ss }} / \sim$ is a geometric invariant theory quotient, hence in particular has a structure of a quasi-projective variety. Let $\mathcal{M}_{\text {quiver }}^{\text {s }}$ denote the open subvariety of $\mathcal{M}_{\text {quiver }}$ given by $\widetilde{\mathcal{M}}$ s $/ \mathcal{G}$. 
Let $\widetilde{\mathcal{M}}_{\text {sym }}$ denote the open subvariety of $\mu_{1}^{-1}(0)$ consisting of points satisfying (S1),(S2), namely we only impose the condition $B_{\zeta_{x}^{+}} A_{x}-A_{x} B_{\zeta_{x}^{-}}+a_{x} b_{x}=0$ and (S1),(S2) for each $x$. We will review that it is a smooth holomorphic symplectic manifold so that the second component $\mu_{2}$ is the moment map with respect to the $\mathcal{G}$-action. (See §3.1.) Therefore $\mathcal{M}$ is a symplectic reduction of $\widetilde{\mathcal{M}}_{\text {sym }}$ by $\mathcal{G}$. In fact, $\widetilde{\mathcal{M}}_{\text {sym }}$ is a product of two way parts $\left(C_{h}, D_{h}\right)$ and triangle parts $\left(A_{x}\right.$, $\left.B_{\zeta_{x}^{ \pm}}, a_{x}, b_{x}\right)$. A two way part is $\operatorname{Hom}\left(V_{\mathrm{o}(h)}, V_{\mathrm{i}(h)}\right) \oplus \operatorname{Hom}\left(V_{\mathrm{i}(h)}, V_{\mathrm{o}(h)}\right)$, which is a symplectic vector space. Moreover $-C_{h} D_{h}$ and $D_{h} C_{h}$ are moment maps for the $\operatorname{GL}\left(V_{\mathrm{i}(h)}\right)$ and $\mathrm{GL}\left(V_{\mathrm{o}(h)}\right)$ action respectively. Therefore it is enough to check the assertion for the triangle part, and we will do in $\S 3.1$.

Now we state an isomorphism between two descriptions.

Theorem 2.1. There is a natural homeomorphism between the space $\mathcal{M}_{\text {bow }}$ of the gauge equivalence classes of bow solutions and the space $\mathcal{M}_{\text {quiver }}$ of the $S$-equivalence classes of quiver representations. Moreover it is an isomorphism of holomorphic symplectic manifolds between the regular locus $\mathcal{M}_{\text {bow }}^{\text {reg }}$ and the stable locus $\mathcal{M}_{\text {quiver }}^{\mathrm{s}}$.

A general theory only guarantees that $\mathcal{M}_{\text {quiver }}^{\mathrm{s}}$ is an orbifold, but we will later show that a point in $\widetilde{\mathcal{M}}_{\text {quiver }}^{\text {s }}$ has only trivial stabilizer (Lemma 2.10). Therefore $\mathcal{M}_{\text {quiver }}^{\mathrm{s}}$ is nonsingular.

This result is, more or less, already known. We first enlarge the gauge equivalence in $\mathcal{M}_{\text {bow }}$ allowing complex gauge not necessarily preserving hermitian inner products. Nahm's equation is divided into real and complex equations correspondingly. The real equation is solved by calculus of variation. Then we solve the complex equation so that the space of complex gauge equivalence classes can be identified with the space of 'boundary conditions'. This step first appeared in [Don84] and subsequently used in works on Nahm's equations [Hur89] and many others.

The space of boundary conditions depends on the situation, but is usually described in terms of Lie-theoretic language such as in [Kro90, Bie97]. For unitary gauge groups in earlier works [Don84, Hur89] and in this paper, they are the space of linear maps instead of Lie-theoretic language.

In [Don84, Hur89], the space of linear maps is further identified with the space of based maps from $\mathbb{P}^{1}$ to a flag variety (of type $A$ ). The second named author in [Tak16] observed that the identification is better understood using the language of quiver, i.e., drawing arrows for linear maps, and applied this idea for Nahm's equation on the circle. This language is useful and inevitable to study complicated situations like in this paper.

The remaining detail of the proof of Theorem 2.1 will be given in $\S 3.3$ below.

We regard the bow variety as $\mathcal{M}_{\text {quiver }}$ equipped with a structure of a quasiprojective algebraic variety hereafter. We drop 'quiver' from the notation and simply denote it by $\mathcal{M}$ hereafter.

When we want to emphasize the parameter $\nu$, we write $\mathcal{M}_{\nu}$.

\subsection{Factorization map}

The factorization map $\Psi: \mathcal{M} \rightarrow \mathbb{A} \mathbf{v}$ is defined as the collection of spectra of $B_{\zeta}$ for various $\zeta$. Although $B_{\zeta}$ is defined for each segment $\zeta, \operatorname{Spec}\left(B_{\zeta}\right)$ and $\operatorname{Spec}\left(B_{\zeta^{\prime}}\right)$ are the same up to constant depending only on parameter $\nu$ when $\zeta$ and $\zeta^{\prime}$ are connected by oriented edges. Therefore we only have $n$ of segments to consider. Hence the vector $\mathbf{v}$ is $\left(\mathbf{v}_{0}, \mathbf{v}_{1}, \ldots, \mathbf{v}_{n-1}\right)$ where $\mathbf{v}_{i}$ is the minimum of dimensions of vector spaces on segments between the $i$ th and $(i+1) x$-points.

We set

$$
|\underline{\mathbf{v}}|=\mathbf{v}_{0}+\mathbf{v}_{1}+\cdots+\mathbf{v}_{n-1}, \quad \mathfrak{S}_{\underline{\mathbf{v}}}=\mathfrak{S}_{\mathbf{v}_{0}} \times \mathfrak{S}_{\mathbf{v}_{1}} \times \cdots \times \mathfrak{S}_{\mathbf{v}_{n-1}}, \quad \mathbb{A} \underline{\mathbf{v}}=\mathbb{A}^{\mid \underline{\mathbf{v}}} \mid \mathfrak{S}_{\underline{\mathbf{v}}} .
$$

\subsection{Stability conditions}

\subsubsection{Characterization of the conditions (S1) and (S2)}

The conditions (S1) and (S2) are not imposed in the original chainsaw variety in [FR14], but will play important role in various contexts of $\mathcal{M}$. We first give other characterizations of the conditions (S1) and (S2). 
Proposition 2.2. The conditions (S1), (S2) is equivalent to $(1,-1)$-semistability for $\mathrm{GL}\left(V_{-}\right) \times$ $\mathrm{GL}\left(V_{+}\right)$-action:

$$
\begin{aligned}
& B_{\zeta^{ \pm}}\left(S_{ \pm}\right) \subset S_{ \pm}, S_{-} \subset \operatorname{Ker} b, A\left(S_{-}\right) \subset S_{+} \Rightarrow \operatorname{dim} S_{-}-\operatorname{dim} S_{+} \leq 0, \\
& B_{\zeta^{ \pm}}\left(T_{ \pm}\right) \subset T_{ \pm}, T_{+} \supset \operatorname{Im} a, A\left(T_{-}\right) \subset T_{+} \Rightarrow \operatorname{codim} T_{-}-\operatorname{codim} T_{+} \geq 0 .
\end{aligned}
$$

Proof. (S1) and (S2) follow from $(1,-1)$-semistability by setting $S_{+}=0, T_{-}=V_{-}$.

Assume $B_{\zeta^{-}}\left(S_{-}\right) \subset S_{-}, S_{-} \subset$ Ker $b$. Then we have $B_{\zeta^{+}} A\left(S_{-}\right) \subset A\left(S_{-}\right)$from $B_{\zeta^{+}} A-A B_{\zeta^{-}}+$ $a b=0$, and each $S_{+}$which satisfies $B_{\zeta^{+}}\left(S_{+}\right) \subset S_{+}$includes $A\left(S_{-}\right)$. Thus the condition (S1) means $\operatorname{dim} S_{-} \leq \operatorname{dim} S_{+}$.

Assume $B_{\zeta^{+}}\left(T_{+}\right) \subset T_{+}, T_{+} \supset \operatorname{Im} a$. Then we have $B_{\zeta^{-}}\left(A^{-1}\left(T_{+}\right)\right) \subset A^{-1}\left(T_{+}\right)$, where $A^{-1}$ means the inverse image, and each $T_{-}$which satisfies $B_{\zeta^{-}}\left(T_{-}\right) \subset T_{-}$includes $A^{-1}\left(T_{+}\right)$. Thus the condition (S2) means $\operatorname{codim} T_{-} \geq \operatorname{codim} T_{+}$.

Remark 2.3. Suppose the bow diagram has one triangle and one two-way parts. Therefore in addition to $B_{ \pm}, A, a, b$ above, we have additional linear maps $C: V_{+} \rightarrow V_{-}, D: V_{-} \rightarrow V_{+}$with extra relations $C D=-B_{-}, D C=-B_{+}$:

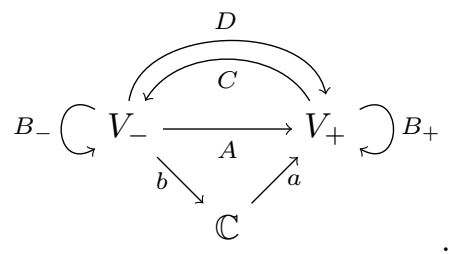

As mentioned in Introduction, the variety of quiver representations describes the moduli space of framed rank 1 perverse coherent sheaves on the blowup $\widehat{\mathbb{P}}^{2}$ [NY11]. In fact, we have choices of stability conditions depending on parameters $\nu_{ \pm}$: if $S_{ \pm} \subset V_{ \pm}$satisfies $C\left(S_{+}\right) \subset S_{-}, D\left(S_{-}\right) \subset S_{+}$, $A\left(S_{-}\right) \subset S_{+}, b\left(S_{-}\right)=0$, we have $\nu_{-} \operatorname{dim} S_{-}+\nu_{+} \operatorname{dim} S_{+} \leq 0$, and similarly for $T_{ \pm} \cdot\left(\nu_{+}, \nu_{-}\right.$are $\zeta_{0}, \zeta_{1}$ in [NY11] respectivelly.) Therefore the above conditions (S1),(S2) imply the semistability with $\nu_{-}=1, \nu_{+}=-1$. But the converse is not true. For example, when $\operatorname{dim} V_{+}=\operatorname{dim} V_{-}=1$, the moduli space in [NY11] with $\nu_{-}=1, \nu_{+}=-1$ is the blowup $\widehat{\mathbb{C}}^{2}$ of the plane. On the other hand, it is easy to check that the bow variety is $\mathbb{C}^{2} .(a=b=0$ in this case.) The difference appears as our conditions imply that $A$ is nonzero, while [NY11] only requires $A, D$ do not vanish simultaneously. Our $\mathbb{C}^{2}$ is the complement of the strict transform of an axis in $\widehat{\mathbb{C}}^{2}$.

Next we consider the following complex on $\mathbb{P}^{1}$ :

$$
V_{-} \otimes \mathcal{O}(-1) \stackrel{\alpha=\left[\begin{array}{c}
x_{1}-x_{0} B_{\zeta^{-}} \\
x_{0} A \\
x_{0} b
\end{array}\right]}{\longrightarrow}\left(V_{-} \oplus V_{+} \oplus \mathbb{C}\right) \otimes \mathcal{O} \stackrel{\beta=\left[-x_{0} A x_{1}-x_{0} B_{\zeta^{+}} x_{0} a\right]}{\longrightarrow} V_{+} \otimes \mathcal{O}(1),
$$

where $\left[x_{0}: x_{1}\right]$ is the homogeneous coordinate on $\mathbb{P}^{1}$. The equation $\mu=0$ means $\beta \alpha=0$.

Proposition 2.4. (i) The condition (S1) is equivalent to injectivity of $\alpha$ at any point of $\mathbb{P}^{1}$. (ii) The condition (S2) is equivalent to surjectivity of $\beta$ at any point of $\mathbb{P}^{1}$.

Proof. For $v \in V_{-}, \alpha v=0$ means $\mathbb{C} v$ is $B_{\zeta^{-}}$-invariant and included in $\operatorname{Ker} A \cap \operatorname{Ker} b$. Thus $\alpha$ is injective if and only if (S1) is satisfied.

As above, a triangle part is closely related to a monad, and this relation leads to Proposition $6.5(2)$.

Let us take a wavy line $\sigma$, and let $x^{1}, \ldots, x^{r}$ be the $x$-points on $\sigma$ in the anticlockwise order. Let $\zeta^{0}, \zeta^{1}, \ldots, \zeta^{r}$ be the corresponding segments. Using Proposition 2.2 successively, we get 
Corollary 2.5. Suppose $S_{\zeta^{i}} \subset V_{\zeta^{i}}\left(\right.$ resp. $\left.T_{\zeta^{i}} \subset V_{\zeta^{i}}\right)$ satisfies

$$
\begin{array}{cccc}
B_{\zeta^{i}}\left(S_{\zeta^{i}}\right) \subset S_{\zeta^{i}} \quad(0 \leq i \leq r), & A_{x^{i}}\left(S_{\zeta^{i-1}}\right) \subset S_{\zeta^{i}}, \quad b_{x^{i}}\left(S_{\zeta^{i-1}}\right)=0 & (1 \leq i \leq r) \\
\left(\text { resp. } B_{\zeta^{i}}\left(T_{\zeta^{i}}\right) \subset T_{\zeta^{i}} \quad(0 \leq i \leq r),\right. & \left.A_{x^{i}}\left(T_{\zeta^{i-1}}\right) \subset T_{\zeta^{i}}, \quad \operatorname{Im} a_{x^{i}} \subset T_{\zeta^{i}} \quad(1 \leq i \leq r)\right) .
\end{array}
$$

Then $\operatorname{dim} S_{\zeta^{0}} \leq \operatorname{dim} S_{\zeta^{1}} \leq \cdots \leq \operatorname{dim} S_{\zeta^{r}}\left(\right.$ resp. $\left.\operatorname{codim} T_{\zeta^{0}} \geq \operatorname{codim} T_{\zeta^{1}} \geq \cdots \geq \operatorname{codim} T_{\zeta^{r}}\right)$.

Remark 2.6. Suppose the bow diagram has only one two-way part. The corresponding quiver appeared in [BF13] and was called the dented chainsaw quiver. The above corollary implies that our data satisfy the stability condition in [BF13], more precisely the $\zeta^{\bullet}$-semistability therein. A slightly stronger stability condition, denoted by $\zeta^{-}$, is introduced so that the dented chainsaw quiver moduli spaces give moduli spaces of framed parabolic sheaves on the blowup $\widehat{\mathbb{P}}^{2}$. It is natural to conjecture that the bow variety $\mathcal{M}$ is the Uhlenbeck-type partial compactification of framed parabolic vector bundles on $\widehat{\mathbb{P}}^{2}$, as $\zeta^{\bullet}$ is on the wall of a chamber where $\zeta^{-}$lives. However $\mathcal{M}$ is not isomorphic to the space $\widehat{\mathfrak{Z}}_{d}$ in [BF13] as the $\zeta^{\bullet}$-semistability and ours are different as we saw in Remark 2.3. Proposition 2.4 suggests that our partial compactification does not allow singularities on parabolic structures.

\subsubsection{Numerical criterion}

We introduce a numerical criterion for the $\nu^{\mathbb{R}_{-}}$(semi) stability.

Definition 2.7. Let $(A, B, a, b, C, D) \in \widetilde{\mathcal{M}}$.

( $\boldsymbol{\nu 1}$ ) Suppose a graded subspace $S=\bigoplus S_{\zeta} \subset \bigoplus V_{\zeta}$ invariant under $A, B, C$, D with $b(S)=0$ is given. We further assume that the restriction of $A$ induces an isomorphism $S_{\zeta^{-}} \stackrel{A}{\cong} S_{\zeta^{+}}$for all $\zeta^{-} x \zeta^{+}$. Then

$$
\sum \nu_{\sigma}^{\mathbb{R}} \operatorname{dim} S_{\sigma} \leq 0
$$

Here $\sigma$ runs over a wavy line, and $\operatorname{dim} S_{\sigma}$ denotes the dimension of the vector space over a segment in $\sigma$. It is independent of the choice of a segment by the assumption.

( $\boldsymbol{\nu 2}$ ) Suppose a graded subspace $T=\bigoplus T_{\zeta} \subset \bigoplus V_{\zeta}$ invariant under $A, B, C$, $D$ with $\operatorname{Im} a \subset T$ is given. We further assume that the restriction of $A$ induces an isomorphism $V_{\zeta^{-}} / T_{\zeta^{-}} \stackrel{A}{\cong}$ $V_{\zeta^{+}} / T_{\zeta^{+}}$for all $\zeta^{ \pm}$as above. Then

$$
\sum \nu_{\sigma}^{\mathbb{R}} \operatorname{codim} T_{\sigma} \geq 0
$$

The sum and $\operatorname{codim} T_{\sigma}$ are as for $(\nu 1)$.

Proposition 2.8. Let $(A, B, a, b, C, D) \in \widetilde{\mathcal{M}}$. Then it is $\nu^{\mathbb{R}}$-semistable if and only if $(\nu 1),(\nu 2)$ are satisfied.

Similarly it is $\nu^{\mathbb{R}_{-}}$stable if and only if we have strict inequalities in $(\nu 1),(\nu 2)$ unless $S_{\zeta}=0$, $T_{\zeta}=V_{\zeta}$ for all $\zeta$.

Proof. Let us follow the argument in [Nak99, Lem. 3.25].

Suppose that $(\nu 1)$ is not satisfied. We take a graded subspace $\bigoplus S_{\zeta}$ satisfying the conditions, but violating the inequality. Then we take complement subspaces $S_{\zeta}^{\perp}$ to $V_{\zeta}$ and define a 1-parameter subgroup $\rho(t)$ by acting $t$ on $S_{\zeta}$ and 1 on $S_{\zeta}^{\perp}$. Since $\chi(g(t))=\prod t^{-\nu_{\sigma}^{\mathbb{R}} \operatorname{dim} S_{\sigma}}$, $\chi(g(t))^{-1}(z) \rightarrow 0$ as $t \rightarrow 0$ as the inequality is violated.

By the assumption the limit of $(A, B, a, b, C, D)$ exists in $\mu^{-1}(0)$. We have a contradiction to the $\nu^{\mathbb{R}}$-semistability condition if we check that the conditions $(\mathrm{S} 1),(\mathrm{S} 2)$ are satisfied for the limit, 
as it means that the limit is in $\widetilde{\mathcal{M}}$. An easy way to check them is to switch to Hurtubise normal form in Proposition 3.2. But let us check them in our description: The limit is the direct sum

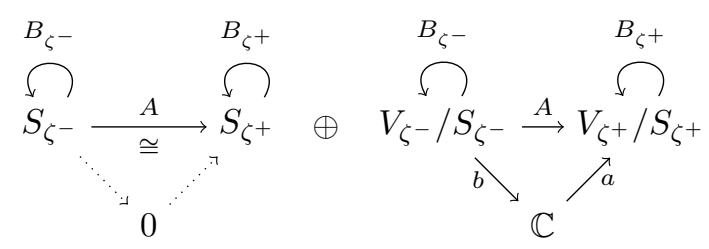

Here we use the same notation for the original linear maps and the induced ones.

Suppose that we are given a subspace $S^{\prime} \subset\left(S_{\zeta^{-}} \oplus V_{\zeta^{-}} / S_{\zeta^{-}}\right)$as in (S1). Then its projection to $V_{\zeta^{-}} / S_{\zeta^{-}}$also satisfies the conditions in (S1) for the second term in (2.9). We take its inverse image $S^{\prime \prime} \subset V_{\zeta^{-}}$. Then $B_{\zeta^{-}}\left(S^{\prime \prime}\right) \subset S^{\prime \prime}, A\left(S^{\prime \prime}\right) \subset S_{\zeta^{+}}$and $b\left(S^{\prime \prime}\right)=0$. Note that $\operatorname{dim} S^{\prime \prime} \geq \operatorname{dim} S_{\zeta^{-}}=$ $\operatorname{dim} S_{\zeta^{+}}$. Proposition 2.2 gives the opposite inequality, therefore $S^{\prime \prime}=S_{\zeta^{-}}$, i.e., the projection of $S^{\prime}$ to $V_{\zeta^{-}} / S_{\zeta^{-}}$is zero. Therefore $S^{\prime}$ is contained in $S_{\zeta^{-}}$. But it means that $S^{\prime}=0$ as $A$ is invertible on $S_{\zeta^{-}}$.

Suppose that we are given a subspace $T^{\prime} \subset\left(S_{\zeta^{+}} \oplus V_{\zeta^{+}} / S_{\zeta^{+}}\right)$as in (S2). Then its projection to $V_{\zeta^{+}} / S_{\zeta^{+}}$also satisfies the conditions in (S2) in the second summand in (2.9). We take its inverse image $T^{\prime \prime} \subset V_{\zeta^{+}}$. Then it satisfies the conditions in (S2) for the original data, hence $T^{\prime \prime}=V_{\zeta^{+}}$. It means that the projection of $T^{\prime}$ to $V_{\zeta^{+}} / S_{\zeta^{+}}$is the whole $V_{\zeta^{+}} / S_{\zeta^{+}}$. But $T^{\prime}$ contains $S_{\zeta^{+}}$as $A: S_{\zeta^{-}} \rightarrow S_{\zeta^{+}}$is an isomorphism. Therefore $T^{\prime}=\left(S_{\zeta^{+}} \oplus V_{\zeta^{+}} / S_{\zeta^{+}}\right)$.

Similarly $(\nu 2)$ follows from the $\nu_{\sigma}$-semistability.

Conversely suppose that $(A, B, a, b, C, D)$ is not $\nu_{\sigma}$-semistable. We take a 1 -parameter subgroup $\rho(t)$ in $\mathcal{G}$ such that $\rho(t) \cdot(A, B, a, b, C, D, z)$ converges to a point in $\widetilde{\mathcal{M}} \times\{0\}$. Consider the weight space decomposition $V_{\zeta}=\bigoplus_{m} V_{\zeta}(m)$, where $\rho(t)$ acts by $t^{m}$ on $V_{\zeta}(m)$. Since $A, B$, $C, D$ converges, the filtration $\cdots \subset F_{\zeta, m}:=\bigoplus_{n \geq m} V_{\zeta}(n) \subset F_{\zeta, m-1}:=\bigoplus_{n \geq m-1} V_{\zeta}(n) \subset \cdots$ is preserved. The limits are the induced maps on the associated graded. Similarly we have $\operatorname{Im} a \subset F_{0}, b\left(F_{1}\right)=0$. The limits of $a, b$ are the induced maps on $F_{0} / F_{1}$, and zero for other components. Thus $B_{\zeta^{+}} A=A B_{\zeta^{-}}$on other components. Then (S1),(S2) for the limit imply $A: F_{\zeta^{-}, m} / F_{\zeta^{-}, m+1} \rightarrow F_{\zeta^{+}, m} / F_{\zeta^{+}, m+1}$ is an isomorphism for $m \neq 0$. Hence $A: F_{\zeta^{-}, m} \rightarrow F_{\zeta^{-}, m}$ is also an isomorphism for $m>0$. Similarly $A: V_{\zeta^{-}} / F_{\zeta^{-}, m} \rightarrow V_{\zeta^{+}} / F_{\zeta^{+}, m}$ is an isomorphism for $m \leq 0$.

We take $F_{\zeta, m}$ with $m>0$ as $S_{\zeta}, F_{\zeta, m}$ with $m \leq 0$ as $T_{\zeta}$. The inequalities in $(\nu 1),(\nu 2)$ are

$$
\sum \nu_{\sigma}^{\mathbb{R}} \operatorname{dim} F_{\sigma, m} \leq 0 \quad \text { for } m>0, \quad \sum \nu_{\sigma}^{\mathbb{R}} \operatorname{codim} F_{\sigma, m} \geq 0 \quad \text { for } m \leq 0
$$

We take the sum over $m \in \mathbb{Z}$ to get

$$
\sum_{\sigma} \sum_{m} \nu_{\sigma} m \operatorname{dim} V_{\sigma}(m) \leq 0
$$

But it is in contradiction with $\lim _{t \rightarrow 0} \chi(\rho(t))^{-1} z=0$.

We leave the proof of the second assertion on the $\nu^{\mathbb{R}}$-stability condition as an exercise for a reader.

Lemma 2.10. If $(A, B, a, b, C, D) \in \widetilde{\mathcal{M}}$ is $\nu^{\mathbb{R}}$-stable, it has a trivial stabilizer.

Proof. Suppose $g \in \mathcal{G}$ stabilizes $(A, B, a, b, C, D)$. Consider $S_{\zeta}=\operatorname{Im}\left(g_{\zeta}-\mathrm{id}\right) \subset V_{\zeta}$ and $T_{\zeta}=$ $\operatorname{Ker}\left(g_{\zeta}-\mathrm{id}\right) \subset V_{\zeta}$. Then the collections $\left\{S_{\zeta}\right\},\left\{T_{\zeta}\right\}$ are invariant under $A, B, C, D$ with $b(S)=0$, $\operatorname{Im} a \subset T$. Assume $S \neq 0$, or equivalently $T \neq V$. By the $\nu^{\mathbb{R}}$-stability, we have

$$
\sum \nu_{\sigma}^{\mathbb{R}} \operatorname{dim} S_{\sigma}<0, \quad \sum \nu_{\sigma}^{\mathbb{R}} \operatorname{codim} T_{\sigma}>0
$$

But this is impossible as codim $T_{\sigma}=\operatorname{dim} S_{\sigma}$. 


\subsection{Tangent space}

Let $\mathbb{M}$ and $\mathbb{N}$ as in $\S 2.2$. Let us consider a three term complex

$$
\bigoplus_{\zeta} \operatorname{End}\left(V_{\zeta}\right) \stackrel{\alpha}{\rightarrow} \mathbb{M} \stackrel{\beta=\left[\begin{array}{l}
\beta_{1} \\
\beta_{2}
\end{array}\right]}{\longrightarrow} \mathbb{N}
$$

$$
\alpha: \bigoplus \xi_{\zeta} \mapsto\left(\begin{array}{c}
{\left[\xi_{\zeta_{x}^{ \pm}}, B_{\zeta_{x}^{ \pm}}\right]} \\
\xi_{\zeta^{+}} A-A \xi_{\zeta^{-}} \\
\xi_{\zeta^{+}} \\
-b \xi_{\zeta^{-}} \\
\xi_{\mathrm{i}(h)} C-C \xi_{\mathrm{o}(h)} \\
\xi_{\mathrm{o}(h)} D-D \xi_{\mathrm{i}(h)}
\end{array}\right), \quad \beta_{1}:\left(\begin{array}{c}
\dot{B} \\
\dot{A} \\
\dot{a} \\
\dot{b} \\
\dot{C} \\
\dot{D}
\end{array}\right) \mapsto B_{\zeta_{x}^{+}} \dot{A}-\dot{A} B_{\zeta_{x}^{-}}+\dot{B}_{\zeta_{x}^{+}} A-A \dot{B}_{\zeta_{x}^{-}}+\dot{a} b+a \dot{b}
$$

and $\beta_{2}$ is

$$
\begin{gathered}
-\dot{B}_{\zeta_{x}^{+}}+\dot{B}_{\zeta_{x^{\prime}}}, \quad \dot{C}_{h} D_{h}+C_{h} \dot{D}_{h}-\dot{D}_{h^{\prime}} C_{h^{\prime}}-D_{h^{\prime}} \dot{C}_{h^{\prime}}, \\
\dot{C}_{h} D_{h}+C_{h} \dot{D}_{h}+\dot{B}_{\zeta_{x}^{-}}, \quad-\dot{B}_{\zeta_{x}^{+}}-\dot{D}_{h} C_{h}-D_{h} \dot{C}_{h},
\end{gathered}
$$

according to two ends of $\zeta$ as before. Note $\alpha$ (resp. $\beta$ ) is the differential of the $\mathcal{G}$-action (resp. $\mu$ ).

Proposition 2.12. (1) $\alpha$ is injective at $(A, B, a, b, C, D) \in \widetilde{\mathcal{M}^{\mathrm{s}}}$.

(2) The first component $\beta_{1}$ of $\beta$ is surjective at $(A, B, a, b, C, D) \in \widetilde{\mathcal{M}}$.

(3) $\beta$ is surjective at $(A, B, a, b, C, D) \in \widetilde{\mathcal{M}^{\mathrm{s}}}$.

Proof. (1) Suppose that $\oplus \xi_{\zeta}$ is in the kernel of $\alpha$. Then $S_{ \pm}:=\operatorname{Im} \xi_{\zeta^{ \pm}}, T_{ \pm}:=\operatorname{Ker} \xi_{\zeta^{ \pm}}$satisfy conditions in Proposition 2.2. Therefore $\operatorname{dim} S_{-} \leq \operatorname{dim} S_{+}, \operatorname{codim} T_{-} \geq \operatorname{codim} T_{+}$. But $\operatorname{codim} T_{ \pm}=\operatorname{dim} S_{ \pm}$. Therefore both must be equality.

Next we consider $S:=\bigoplus \operatorname{Im} \xi_{\zeta}, T:=\bigoplus \operatorname{Ker} \xi_{\zeta}$. The conditions in $(\nu 1),(\nu 2)$ are satisfied, hence the same argument shows $\sum \nu_{\sigma}^{\mathbb{R}} \operatorname{dim} S_{\sigma}=0$, and hence $S=0$, i.e., $\xi_{\zeta}=0$ for all $\zeta$.

(2) Suppose $\oplus \eta_{x}$ is perpendicular to the image of the first component of $\beta$ with respect to the trace pairing. Then

$$
A \eta_{x}=0=\eta_{x} A, \quad B_{\zeta^{-}} \eta_{x}=\eta_{x} B_{\zeta^{+}}, \quad b \eta_{x}=0=\eta_{x} a .
$$

Then $S:=\operatorname{Im} \eta_{x}$ is zero by (S1). (Or $T:=\operatorname{Ker} \eta_{x}$ is $V_{2}$ by (S2).) Therefore $\eta_{x}=0$.

(3) Suppose $\oplus \eta_{x} \oplus \xi_{\zeta}$ is perpendicular to the image of $\beta$ with respect to the trace pairing. Then

$$
B_{\zeta^{-}} \eta_{x}=\eta_{x} B_{\zeta^{+}}, \quad b \eta_{x}=0=\eta_{x} a, \quad A \eta_{x}=\xi_{\zeta^{+}}, \quad \eta_{x} A=\xi_{\zeta^{-}},
$$

and

$$
C \xi_{\mathrm{o}(h)}=\xi_{\mathrm{i}(h)} C, \quad D \xi_{\mathrm{i}(h)}=\xi_{\mathrm{o}(h)} D .
$$

Note that $B_{\zeta^{-}} \eta_{x} A=\eta_{x} A B_{\zeta^{-}}$and $B_{\zeta^{+}} A \eta_{x}=A \eta_{x} B_{\zeta^{+}}$. Therefore the same argument as in (1) shows $\xi_{\zeta}=0$ for all $\zeta$. Next we consider $S:=\operatorname{Im} \eta_{x} \subset V_{\zeta^{-}}$. Then the condition for (S1) is satisfied. Hence we have $S=0$, i.e., $\eta_{x}=0$.

By $(3), \widetilde{\mathcal{M}}$ is smooth at $(A, B, a, b, C, D) \in \widetilde{\mathcal{M}^{\mathrm{s}}}$. The tangent space is isomorphic to Ker $\beta$. On the stable locus, the quotient map $\widetilde{\mathcal{M}^{\mathrm{s}}} \rightarrow \mathcal{M}^{\mathrm{s}}$ is a principal $\mathcal{G}$-bundle. Hence the tangent space of the quotient $\mathcal{M}^{\mathrm{s}}$ is isomorphic to $\operatorname{Ker} \beta / \operatorname{Im} \alpha$. By $(2) \widetilde{\mathcal{M}}_{\text {sym }}$ is smooth at $(A, B, a, b, C, D) \in$ $\widetilde{\mathcal{M}}$. The tangent space is isomorphic to $\operatorname{Ker} \beta_{1}$. (Recall $\widetilde{\mathcal{M}}_{\text {sym }}$ is the open subvariety of $\mu_{1}^{-1}(0)$ consisting of points satisfying $(\mathrm{S} 1),(\mathrm{S} 2)$.)

In particular, we have

Proposition 2.13. The (possibly empty) open locus $\mathcal{M}^{\mathrm{s}}$ is smooth of dimension $\operatorname{dim} \mathbb{M}-\operatorname{dim} \mathbb{N}-$ $\operatorname{dim} \mathcal{G}$. 


\subsection{Balanced and cobalanced conditions}

We define $N_{h}:=\operatorname{dim} V_{\mathrm{i}(h)}-\operatorname{dim} V_{\mathrm{o}(h)}$ for an arrow $h$ and $N_{x}:=\operatorname{dim} V_{\zeta^{-}}-\operatorname{dim} V_{\zeta^{+}}$for $x \in \Lambda$, where $\zeta^{ \pm}$are the adjacent segments $\begin{aligned} & \zeta^{-} x \zeta^{+} \\ & \sim x \sim\end{aligned}$. Of course, we mean $\left|N_{x}\right|=\Delta R(x)$.

Definition 2.14. A dimension vector $\underline{\mathbf{v}}$ is called balanced if $N_{h}=0$ for each $h$. A dimension vector $\underline{\mathbf{v}}$ is called cobalanced if $N_{x}=0$ for each $x$.

The balanced condition will play an important role in $\S 6$. For the cobalanced condition we have

Theorem 2.15 ([Che11]). Consider a bow diagram with a cobalanced dimension vector. Assume there is at least one oriented edge. Then the corresponding bow variety $\mathcal{M}$ is isomorphic to a quiver variety $\mathfrak{M}$ in [Nak94b] as an affine algebraic variety. Moreover the isomorphism respects the symplectic form on regular locus. Conversely, a quiver variety of an affine type $A$ is described as a bow variety with a cobalanced dimension vector.

A quiver variety $\mathfrak{M}$ is a symplectic reduction of $\mathbf{N} \oplus \mathbf{N}^{*}$ by $G=\prod \mathrm{GL}\left(\mathbf{v}_{i}\right)$, where $\mathbf{N}$ is as in Introduction. The level of the moment map is $\nu^{\mathbb{C}}$, and the quotient is the geometric invariant theory quotient with the $\nu^{\mathbb{R}}$-semistability.

Proof. This is the case stated in (c) of $\S 2.1$. In the quiver description, $\operatorname{dim} V_{\zeta^{-}}=\operatorname{dim} V_{\zeta^{+}}$. Then $A$ is an isomorphism by Lemma 3.1 below. We eliminate Nahm's data on segments, or $A$ in the quiver description, and $(I, J)$ forms a framing part of a quiver variety. $\mathrm{o}\left(h^{\prime}\right)$.

For example, consider the case when $r_{\sigma}=1, \mathrm{i}(h)=x_{\sigma, L}, \zeta^{-}=\left[x_{\sigma, L}, x_{\sigma}\right], \zeta^{+}=\left[x_{\sigma}, x_{\sigma, R}\right], x_{\sigma, R}=$

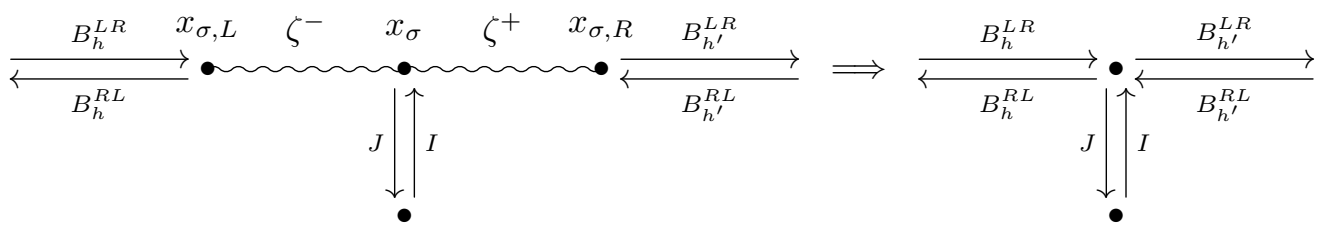

The segments $\zeta^{ \pm}$give the cotangent bundle $T^{*} \mathrm{GL}\left(V_{\zeta^{ \pm}}\right)$by Kronheimer [Kro88]. In the quiver description, we use Proposition 3.2(1) below. Hence we can eliminate them in the symplectic quotient by imposing the equation

$$
B_{h}^{L R} B_{h}^{R L}-B_{h^{\prime}}^{R L} B_{h^{\prime}}^{L R}+I J=\left(\nu_{\sigma}^{2}+\sqrt{-1} \nu_{\sigma}^{3}\right) \mathrm{id}
$$

from 3.(a) and (c) in $§ 2.1$, and so on. This is nothing but the defining equation in [Nak94b].

\section{Constituents of bow varieties}

In this section, we study properties of triangle and two-way parts in the quiver description of a bow variety. We need these properties in later sections, in particular in $\S 6$ to prove a balanced bow variety is a Coulomb branch of quiver gauge theory of affine type $A$. 


\subsection{Triangle part}

\subsubsection{Definition}

Consider

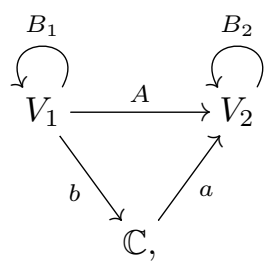

where $\operatorname{dim} V_{k}=\mathbf{v}_{k}$. Set

$$
\begin{aligned}
\mathbb{M} & :=\operatorname{Hom}\left(V_{1}, V_{2}\right) \oplus \operatorname{End} V_{1} \oplus \operatorname{End} V_{2} \oplus \operatorname{Hom}\left(\mathbb{C}, V_{2}\right) \oplus \operatorname{Hom}\left(V_{1}, \mathbb{C}\right), \\
\operatorname{GL}(\underline{\mathbf{v}}) & :=\operatorname{GL}\left(V_{1}\right) \times \operatorname{GL}\left(V_{2}\right), \\
\widetilde{\mathcal{M}} & :=\left\{\left(A, B_{1}, B_{2}, a, b\right) \in \mu^{-1}(0) \mid\left(A, B_{1}, B_{2}, a, b\right) \text { satisfies (S1) and }(\mathrm{S} 2)\right\}, \\
\mathcal{M} & :=[\widetilde{\mathcal{M}} / \mathrm{GL}(\underline{\mathbf{v}})] \quad \text { (quotient stack). }
\end{aligned}
$$

Here,

$$
\begin{aligned}
\mu\left(A, B_{1}, B_{2}, a, b\right) & :=B_{2} A-A B_{1}+a b, \\
(\mathrm{~S} 1) & : B_{1}\left(S_{1}\right) \subset S_{1}, S_{1} \subset \operatorname{Ker} A \cap \operatorname{Ker} b \Rightarrow S_{1}=0, \\
(\mathrm{~S} 2) & : B_{2}\left(T_{2}\right) \subset T_{2}, T_{2} \supset \operatorname{Im} A+\operatorname{Im} a \Rightarrow T_{2}=V_{2},
\end{aligned}
$$

and the $\mathrm{GL}(\underline{\mathbf{v}})$-action is

$$
\left(A, B_{1}, B_{2}, a, b\right) \mapsto\left(g_{2} A g_{1}^{-1}, g_{1} B_{1} g_{1}^{-1}, g_{2} B_{2} g_{2}^{-1}, g_{2} a, b g_{1}^{-1}\right), \quad\left(g_{1}, g_{2}\right) \in \mathrm{GL}(\underline{\mathbf{v}}) .
$$

By Proposition 2.12(2) $\widetilde{\mathcal{M}}$ is a $\left(\mathbf{v}_{1}^{2}+\mathbf{v}_{1}+\mathbf{v}_{2}^{2}+\mathbf{v}_{2}\right)$-dimensional smooth variety. We will use the quotient stack $\mathcal{M}$ just to reduce notational redundancy in the factorization (Proposition 3.6 below). One can simply understand $[X / G] \cong[Y / H]$ as a $G$-equivariant isomorphism $X \cong Y \times{ }_{H} G$. (Here $H$ is a subgroup of $G$.)

For $\widetilde{\mathcal{M}}$, the following results play important roles.

Lemma 3.1 ([Tak16, Lem. 2.18]). A has full rank.

We consider solutions of Nahm's equations on an interval, which contains a single $x$-point. The solutions extend smoothly at the end points. We consider a gauge transformation $u$ as before, but we impose the condition that $u$ is the identity at the end points.

Proposition 3.2. (1) [Hur89, Prop. $1.15+\S 2]$ The moduli space of solutions of Nahm's equations over the interval is isomorphic to the space of matrices of forms

$$
\begin{aligned}
u \in \operatorname{GL}(n), \eta= & {\left[\begin{array}{ccc}
h & 0 & g \\
f & 0 & e_{0} \\
0 & \text { id } & e
\end{array}\right] \in \mathfrak{g l}(n), } \\
& \left(e_{0}, e, f, g, h\right) \in \mathbb{C} \times \mathbb{C}^{n-m-1} \times\left(\mathbb{C}^{m}\right)^{*} \times \mathbb{C}^{m} \times \operatorname{End}\left(\mathbb{C}^{m}\right),
\end{aligned}
$$

for $n=\max \left(\mathbf{v}_{1}, \mathbf{v}_{2}\right), m=\min \left(\mathbf{v}_{1}, \mathbf{v}_{2}\right)$ when $\mathbf{v}_{1} \neq \mathbf{v}_{2}$, and

$$
u \in \operatorname{GL}(n), h \in \mathfrak{g l}(n), I \in \mathbb{C}^{n}, J \in\left(\mathbb{C}^{n}\right)^{*}
$$

when $n=\mathbf{v}_{1}=\mathbf{v}_{2}$. The action is given by

$$
\begin{gathered}
(u, \eta) \mapsto\left(\left[\begin{array}{ccc}
g_{m} & 0 & 0 \\
0 & \text { id } & 0 \\
0 & 0 & \text { id }
\end{array}\right] u g_{n}^{-1},\left[\begin{array}{ccc}
g_{m} & 0 & 0 \\
0 & \text { id } & 0 \\
0 & 0 & \text { id }
\end{array}\right] \eta\left[\begin{array}{ccc}
g_{m}^{-1} & 0 & 0 \\
0 & \text { id } & 0 \\
0 & 0 & \text { id }
\end{array}\right]\right), \quad\left(g_{n}, g_{m}\right) \in \operatorname{GL}(n) \times \operatorname{GL}(m) \quad \text { if } \mathbf{v}_{1} \neq \mathbf{v}_{2}, \\
(u, h, I, J) \mapsto\left(g_{2} u g_{1}^{-1}, g_{2} h g_{2}^{-1}, g_{2} I, J g_{2}^{-1}\right), \quad\left(g_{1}, g_{2}\right) \in \mathrm{GL}(\underline{\mathbf{v}}) \quad \text { if } \mathbf{v}_{1}=\mathbf{v}_{2} .
\end{gathered}
$$


(2) [Tak16, Prop. 2.9] The above space of matrices is isomorphic to $\widetilde{\mathcal{M}}$ by

$$
\left(A, B_{1}, B_{2}, a, b\right)= \begin{cases}\left(\left[\begin{array}{ll}
\text { id } & 0
\end{array}\right] u, u^{-1} \eta u, h, g,\left[\begin{array}{ll}
0 & 0
\end{array}\right] u\right), & \mathbf{v}_{1}>\mathbf{v}_{2}, \\
\left(u, u^{-1} h u, h-I J, I, J u\right), & \mathbf{v}_{1}=\mathbf{v}_{2}, \\
\left(-u^{-1}\left[\begin{array}{c}
\text { id } \\
0
\end{array}\right],-h,-u^{-1} \eta u, u^{-1}\left[\begin{array}{l}
0 \\
1 \\
0
\end{array}\right],-f\right), & \mathbf{v}_{1}<\mathbf{v}_{2}\end{cases}
$$

Notice that the matrix $\left[\begin{array}{lll}0 & 0 & 0 \\ 0 & 0 & 0 \\ 0 & \text { id } & 0\end{array}\right]$ is nilpotent, and when we regard this nilpotent matrix as $Y$ of an $\mathfrak{s l}(2)$-triple $\{H, X, Y\},\left[\begin{array}{lll}0 & 0 & 1\end{array}\right]$ is the highest weight covector and $\left[\begin{array}{l}0 \\ 1 \\ 0\end{array}\right]$ is the lowest weight vector.

A pair of matrices $u, \eta$ (and with $I, J$ when $\mathbf{v}_{1}=\mathbf{v}_{2}$ ) as above is called Hurtubise normal form. As we mentioned after Theorem 2.1, (1) was proved in two steps: the first step solves the real equation by calculus of variation. The second step is the classification of the solution of the complex equation.

By combining two isomorphisms, we see that $\widetilde{\mathcal{M}}$ is isomorphic to a moduli space of solutions of Nahm's equations over an interval, and has a hyper-Kähler structure. Note also that $\widetilde{\mathcal{M}}$ is an affine algebraic variety, as the space of Hurtubise normal forms is a product of a general linear group and an affine space.

Corollary 3.3. The holomorphic symplectic form on $\widetilde{\mathcal{M}}$ is given in the Hurtubise normal form by

$$
\omega= \begin{cases}\operatorname{tr}\left(d \eta \wedge d u u^{-1}+\eta d u u^{-1} \wedge d u u^{-1}\right) & \text { when } \mathbf{v}_{1} \neq \mathbf{v}_{2} \\ \operatorname{tr}\left(d h \wedge d u u^{-1}+h d u u^{-1} \wedge d u u^{-1}+d I \wedge d J\right) & \text { when } \mathbf{v}_{1}=\mathbf{v}_{2}\end{cases}
$$

In case $\mathbf{v}_{1}=\mathbf{v}_{2}$, the above is the standard symplectic form on $T^{*} \operatorname{GL}(n) \times \mathbb{C}^{n} \times\left(\mathbb{C}^{n}\right)^{*}$. When $\mathbf{v}_{1} \neq \mathbf{v}_{2}$, this is a consequence of [Bie97]. More precisely, we can apply [Bie97] by the following remark.

Remark 3.4. When $\mathbf{v}_{1} \neq \mathbf{v}_{2}$, we can forget Nahm's data on the interval for the smaller rank. Then we have $\widetilde{\mathcal{M}} \cong \mathrm{GL}(n) \times \mathcal{S}$, where $\mathcal{S}$ is the Slodowy transversal slice of the nilpotent element $\left[\begin{array}{lll}0 & 0 & 0 \\ 0 & 0 & 0 \\ 0 & \text { id } & 0\end{array}\right]$ by [Bie97]. However the space of Hurtubise normal forms is not the Slodowy slice defined by $Y+\mathfrak{z}_{\mathfrak{g}}(X)$ : Consider an $\mathfrak{s l}(2)$-triple

$$
\begin{aligned}
& X:=\left[\begin{array}{c|c}
0 & 0 \\
\hline 0 & X_{0}
\end{array}\right], X_{0}:=\left(\begin{array}{ccccc}
0 & n^{\prime}-1 & & & 0 \\
& & 2\left(n^{\prime}-2\right) & & \\
& & & \ddots & \\
& & & & n^{\prime}-1 \\
& 0 & & & 0
\end{array}\right), \\
& Y:=\left[\begin{array}{c|c}
0 & 0 \\
\hline 0 & Y_{0}
\end{array}\right], Y_{0}:=\left(\begin{array}{ccccc}
0 & & & & \\
1 & & & 0 & \\
& & \ddots & & \\
& 0 & & & \\
& 0 & & 1 & 0
\end{array}\right), \\
& H:=\left[\begin{array}{c|c}
0 & 0 \\
\hline 0 & H_{0}
\end{array}\right], H_{0}:=\left(\begin{array}{cccc}
n^{\prime}-1 & & 0 & 0 \\
& n^{\prime}-3 & & \\
& & \ddots & \\
0 & & & 1-n^{\prime}
\end{array}\right) \text {, }
\end{aligned}
$$


where $n^{\prime}=n-m$. Then a matrix in $Y+\mathfrak{z}_{\mathfrak{g}}(X)$ is of the form

$$
Y+\left(\begin{array}{c|cccc}
h & 0 & \cdots & 0 & g \\
\hline f & & & & \\
0 & & & P \\
\vdots & & & P \\
0 & & &
\end{array}\right) \quad\left(P \in \mathbb{C}\left[X_{0}\right]\right) .
$$

Nevertheless the proof of [Bie97] works for the space of Hurtubise normal forms: The space of solutions of Nahm's equation is first identified with a symplectic quotient of $T^{*} \mathrm{GL}(n)$ by a certain nilpotent subgroup of $\mathrm{GL}(n)$. Then the latter is shown to be isomorphic to $\mathrm{GL}(n) \times \mathcal{S}$. The second statement is [Bie97, Lem. 3.2], but the same proof works also for Hurtubise normal forms.

The moment map for the $\mathrm{GL}\left(\mathbf{v}_{1}\right) \times \mathrm{GL}\left(\mathbf{v}_{2}\right)$-action is $\left(-B_{1}, B_{2}\right)$ by the formula [Bie97, (2.11)].

Corollary 3.5. There exists a $\mathrm{GL}\left(\mathbf{v}_{1}\right) \times \mathrm{GL}\left(\mathbf{v}_{2}\right)$-equivariant isomorphism between a triangle and its inverse-orientation:

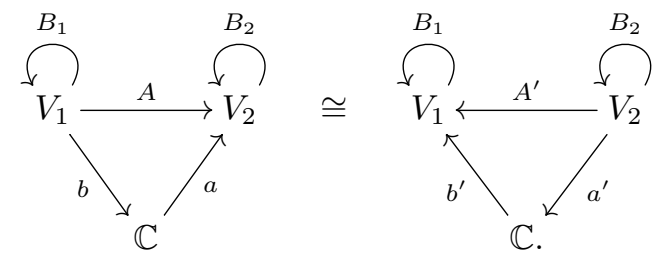

Proof. By Proposition 3.2, triangles in the both sides are isomorphic to the same moduli space of solutions of Nahm's equations over an interval.

More concretely it is given by

$$
A^{\prime}=A^{-1}, \quad a^{\prime}=b A^{-1}, \quad b^{\prime}=A^{-1} a
$$

if $\mathbf{v}_{1}=\mathbf{v}_{2}$, and through $(u, \eta)$ if $\mathbf{v}_{1} \neq \mathbf{v}_{2}$.

\subsubsection{Factorization}

Let $\Psi$ be a map from a triangle to eigenvalues $w_{1, k}$ of $B_{1}$ and $w_{2, k}$ of $B_{2}$ :

$$
\Psi: \mu^{-1}(0) \rightarrow \mathbb{A} \mathbf{v} .
$$

Here $\mathbb{A} \underline{\mathbf{v}}=\mathbb{A}|\mathbf{v}| / \mathfrak{S}_{\mathbf{v}}=S^{\mathbf{v}_{1}} \mathbb{C} \times S^{\mathbf{v}_{2}} \mathbb{C}$ as in $\S 2.3$. For $\underline{\mathbf{v}}=\underline{\mathbf{v}}^{\prime}+\underline{\mathbf{v}}^{\prime \prime}$, let $\left(\mathbb{A}^{\underline{\mathbf{v}}^{\prime}} \times \mathbb{A}^{\underline{\mathbf{v}}^{\prime \prime}}\right)$ disj be the open subset of $\mathbb{A} \underline{\mathbf{v}}^{\prime} \times \mathbb{A} \underline{v}^{\prime \prime}$ consisting of pairs of disjoint configurations.

Proposition 3.6 (cf. [FR14, Proof of Prop. 2.11]). $\mathcal{M}$ has a factorization isomorphism:

$$
\mathfrak{f}_{\underline{\mathbf{v}}^{\prime}, \mathbf{v}^{\prime \prime}}: \mathcal{M}(\underline{\mathbf{v}}) \times_{\mathbb{A} \underline{\mathbf{v}}}\left(\mathbb{A}^{\mathbf{v}^{\prime}} \times \mathbb{A} \underline{\mathbf{v}}^{\prime \prime}\right)_{\text {disj }} \stackrel{\sim}{\longrightarrow}\left(\mathcal{M}\left(\underline{\mathbf{v}}^{\prime}\right) \times \mathcal{M}\left(\underline{\mathbf{v}}^{\prime \prime}\right)\right) \times_{\mathbb{A} \mathbf{v}^{\prime}} \times \mathbb{A} \mathbf{v}^{\prime \prime}\left(\mathbb{A} \underline{\mathbf{v}}^{\prime} \times \mathbb{A}^{\underline{\mathbf{v}}^{\prime \prime}}\right)_{\text {disj }}
$$

We use the next obvious lemma:

Lemma 3.7. Let $J(w, n)$ be the Jordan block with size $n$ and eigenvalue $w$, and $\phi_{\left(n, m, w_{1}, w_{2}\right)}$ be a linear map

$$
\phi_{\left(n, m, w_{1}, w_{2}\right)}: M(n, m ; \mathbb{C}) \rightarrow M(n, m ; \mathbb{C}), \quad \phi_{\left(n, m, w_{1}, w_{2}\right)}(A)=J\left(w_{1}, n\right) A-A J\left(w_{2}, m\right) .
$$

(i) When $w_{1} \neq w_{2}$, this map is an isomorphism.

(ii) When $w_{1}=w_{2}, \operatorname{rank} \phi_{(n, m, w, w)}=m n-\min (n, m)$. 
Proof of Proposition 3.6. The following is the same as one in [FR14], but we check also that the isomorphism preserves the conditions $(\mathrm{S} 1)$ and $(\mathrm{S} 2)$. Let us decompose $V_{i}=V_{i}^{(1)} \oplus V_{i}^{(2)}$, where $V_{i}^{(1)}$ is the eigenspace of $B_{i}$ with eigenvalue $w \in \mathbb{C}$, and $V_{i}^{(2)}$ is the sum of the eigenspaces with different eigenvalues. We take a representative

$$
\left(A, B_{1}, B_{2}, a, b\right)=\left(\left[\begin{array}{ll}
A^{(11)} & A^{(12)} \\
A^{(21)} & A^{(22)}
\end{array}\right],\left[\begin{array}{cc}
B_{1}^{(1)} & 0 \\
0 & B_{1}^{(2)}
\end{array}\right],\left[\begin{array}{cc}
B_{2}^{(1)} & 0 \\
0 & B_{2}^{(2)}
\end{array}\right],\left[\begin{array}{l}
a^{(1)} \\
a^{(2)}
\end{array}\right],\left[\begin{array}{ll}
b^{(1)} & b^{(2)}
\end{array}\right]\right) .
$$

Let $S_{1}^{(1)}$ be a subspace of $V_{1}^{(1)}$ such that $B_{1}^{(1)} S_{1}^{(1)} \subset S_{1}^{(1)}$ and $S_{1}^{(1)} \subset \operatorname{Ker} A^{(11)} \cap \operatorname{Ker} b^{(1)}$. By the equation, for any $v \in S_{1}^{(1)}$ and $l \in \mathbb{Z}_{\geq 0}$, we have

$$
\left(B_{2}^{(2)}-w \mathrm{id}\right)^{l} A^{(21)} v=A^{(21)}\left(B_{1}^{(1)}-w \mathrm{id}\right)^{l} v .
$$

On the other hand, we get $\left(B_{1}^{(1)}-w \text { id }\right)^{l}=0$ for some $l$. Since eigenvalues of $B_{1}^{(1)}$ and $B_{2}^{(2)}$ are disjoint, $\left(B_{2}^{(2)}-w \mathrm{id}\right)^{l}$ becomes invertible for any $l$. Thus $A^{(21)} S_{1}^{(1)}=0$ holds. Then $S_{1}^{(1)}=0$ follows from $(\mathrm{S} 1)$ for a whole triangle, and this means that $\left(A^{(11)}, B_{1}^{(1)}, B_{2}^{(1)}, a^{(1)}, b^{(1)}\right)$ satisfies (S1). One can check that $\left(A^{(11)}, B_{1}^{(1)}, B_{2}^{(1)}, a^{(1)}, b^{(1)}\right)$ satisfies (S2) by the similar argument.

Furthermore, $A^{(21)}$ satisfies the equation $B_{2}^{(2)} A^{(21)}-A^{(21)} B_{1}^{(1)}+a^{(2)} b^{(1)}=0$, and determined as $\phi_{\left(\operatorname{dim} V_{2}^{(2)}, \operatorname{dim} V_{1}^{(1)}, w_{1}, w_{2}\right)}^{-1}\left(a^{(2)} b^{(1)}\right)$ under this assumption by Lemma 3.7. Thus, we get a map from the right hand side to the left hand side:

$$
\widetilde{\mathcal{M}}(\underline{\mathbf{v}}) \times_{\mathbb{A} \underline{\mathbf{v}}}\left(\mathbb{A}^{\underline{\mathbf{v}}^{\prime}} \times \mathbb{A} \underline{\mathbf{v}}^{\prime \prime}\right)_{\operatorname{disj}} \leftarrow\left(\widetilde{\mathcal{M}}\left(\underline{\mathbf{v}}^{\prime}\right) \times \widetilde{\mathcal{M}}\left(\underline{\mathbf{v}}^{\prime \prime}\right)\right) \times_{\mathbb{A} \underline{\mathbf{v}}^{\prime} \times \mathbb{A} \underline{\mathbf{v}}^{\prime \prime}}\left(\mathbb{A} \underline{\mathbf{v}}^{\prime} \times \mathbb{A} \underline{\mathbf{v}}^{\prime \prime}\right)_{\operatorname{disj}},
$$

equivariant under $\mathrm{GL}\left(\underline{\mathbf{v}}^{\prime}\right) \times \mathrm{GL}\left(\underline{\mathbf{v}}^{\prime \prime}\right)$, which induces an isomorphism of stacks.

Let $\AA|\underline{\mathbf{v}}|$ be the open subset of $\mathbb{A}|\underline{\mathbf{v}}|$ consisting of distinct configurations, and define $\AA \underline{\mathbf{v}}:=$

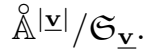

Corollary 3.8. $\widetilde{\mathcal{M}} \times_{\AA \underline{\mathbf{v}}} \AA|\underline{\mathbf{v}}| \cong \AA|\underline{\mathbf{v}}| \times \mathrm{GL}(\underline{\mathbf{v}})$.

Proof. We order eigenvalues of $B_{1}$ and $B_{2}$ some way, and denote them by $\left(w_{1,1}, w_{1,2}, \cdots\right)$ and $\left(w_{2,1}, w_{2,2}, \cdots\right)$ respectively. Since they are distinct, we have $w_{i, k} \neq w_{j, l}$ if $(i, k) \neq(j, l)$. By using the above proposition repeatedly, we can take a representative of $\left(A, B_{1}, B_{2}, a, b\right)$ as

$$
A^{(k l)}=\left(w_{1, l}-w_{2, k}\right)^{-1}, B_{1}=\operatorname{diag}\left(w_{1, k}\right), B_{2}=\operatorname{diag}\left(w_{2, k}\right), a={ }^{t}[1, \cdots, 1], b=[1, \cdots, 1] .
$$

On this representative, $\mathrm{GL}(\underline{\mathbf{v}})$ acts freely.

Corollary 3.9. $\mu^{-1}(0) \times_{\AA \underline{\mathbf{v}}} \AA|\underline{\mathbf{v}}| \cong \AA|\underline{\mathbf{v}}| \times\left(\mathrm{GL}(\underline{\mathbf{v}}) \times \mathbb{C}^{|\underline{\mathbf{v}}|}\right) /\left(\mathbb{C}^{\times}\right)^{|\underline{\mathbf{v}}|}$.

\subsubsection{Dimension estimate for $\Psi^{-1}$}

Next we calculate the dimension of the fiber of $\Psi$. Though the following proposition is in [FR14, Prop. 2.11], we give a self-contained argument for the sake of a reader.

Proposition 3.10. Over each point of $\mathbb{A} \mathbf{v}$, the dimension of the fiber of $\Psi$ is $\mathbf{v}_{1}^{2}+\mathbf{v}_{2}^{2}$.

Proof. For $\AA$ 느, the assertion follows from Corollary 3.9.

We consider the fiber of $0 \in \mathbb{A} \underline{\mathbf{v}}$. Suppose $B_{1}$ and $B_{2}$ are regular nilpotent. We make both $B_{1}$ and $B_{2}$ Jordan normal forms. Then $B_{1} A-A B_{2}$ has the following property: the sum of all elements in the $i$ th diagonal, counting from the lower left corner, is 0 for $i=1, \ldots, \min \left(\mathbf{v}_{1}, \mathbf{v}_{2}\right)$. Thus $\operatorname{dim}\{(a, b)\}$ is at most $\mathbf{v}_{1}+\mathbf{v}_{2}-\min \left(\mathbf{v}_{1}, \mathbf{v}_{2}\right)=\max \left(\mathbf{v}_{1}, \mathbf{v}_{2}\right)$. Once $B_{1}, B_{2}, a, b$ are fixed, the space of solutions of the linear equation $\{A\}=\phi_{\left(\mathbf{v}_{1}, \mathbf{v}_{2}, 0,0\right)}^{-1}(a b)$ has $\min \left(\mathbf{v}_{1}, \mathbf{v}_{2}\right)$-dimension. Lemma 3.7 also says that $\operatorname{dim} \mathrm{GL}_{B_{k}}=\mathbf{v}_{k}$. Thus we have

$$
\operatorname{dim}\left(\Psi^{-1}(0) \cap\left\{B_{k}=J\left(0, \mathbf{v}_{k}\right)\right\}\right)=\max \left(\mathbf{v}_{1}, \mathbf{v}_{2}\right)+\min \left(\mathbf{v}_{1}, \mathbf{v}_{2}\right)+\mathbf{v}_{1}^{2}+\mathbf{v}_{2}^{2}-\mathbf{v}_{1}-\mathbf{v}_{2}=\mathbf{v}_{1}^{2}+\mathbf{v}_{2}^{2}
$$


When $B_{1}$ and $B_{2}$ are not regular nilpotent, we describe them as length $l$ partition: $B_{1}=$ $\left[m_{1}, \cdots, m_{l}\right], B_{2}=\left[n_{1}, \cdots, n_{l}\right], \sum m_{i}=\mathbf{v}_{1}, \sum n_{i}=\mathbf{v}_{2}$. We have the corresponding block decompositions $\left(A_{i j}\right),\left(a_{i}\right),\left(b_{i}\right)$ of $A, a, b$. From the above argument for the equation for $a_{i}, b_{i}$, we have $\operatorname{dim}\{(a, b)\} \leq \sum_{i} \max \left(m_{i}, n_{i}\right)$. We also have $\operatorname{dim}\{A\}=\sum_{i, j} \min \left(m_{i}, n_{j}\right)$ and $\operatorname{dim} \mathrm{GL}_{B_{1}}=\sum_{i, j} \min \left(m_{i}, m_{j}\right)$. Thus,

$$
\begin{aligned}
\operatorname{dim}\left(\Psi^{-1}(0) \cap\right. & \left.\left\{B_{k} \neq J\left(0, \mathbf{v}_{k}\right)\right\}\right) \\
& \leq \sum_{i} \max \left(m_{i}, n_{i}\right)+\sum_{i, j}\left\{\min \left(m_{i}, n_{j}\right)-\min \left(m_{i}, m_{j}\right)-\min \left(n_{i}, n_{j}\right)\right\}+\mathbf{v}_{1}^{2}+\mathbf{v}_{2}^{2} \\
& =\sum_{i \neq j}\left\{\min \left(m_{i}, n_{j}\right)-\min \left(m_{i}, m_{j}\right)-\min \left(n_{i}, n_{j}\right)\right\}+\mathbf{v}_{1}^{2}+\mathbf{v}_{2}^{2} \leq \mathbf{v}_{1}^{2}+\mathbf{v}_{2}^{2} .
\end{aligned}
$$

Thus we have $\operatorname{dim}\left(\Psi^{-1}(0)\right)=\mathbf{v}_{1}^{2}+\mathbf{v}_{2}^{2}$. For other fibers, we use the factorization.

Corollary 3.11. $\mu^{-1}(0)$ is an irreducible reduced complete intersection in $\mathbb{M}$.

Proof. $\Psi^{-1}(\AA \underline{\mathbf{v}})$ is smooth and the dimension of its complement is less than $\mathbf{v}_{1}^{2}+\mathbf{v}_{1}+\mathbf{v}_{2}^{2}+\mathbf{v}_{2}$.

\subsubsection{Shift}

Note

$$
\mu\left(A, B_{1}, B_{2}, a, b\right)=\left(B_{2}+\zeta \operatorname{id}_{V_{2}}\right) A-A\left(B_{1}+\zeta \operatorname{id}_{V_{1}}\right)+a b .
$$

Therefore we have an action of $\mathbb{C}$ on $\mu^{-1}(0)$ and $\widetilde{\mathcal{M}}$ so that the moment map is shifted as $\left(-B_{1}, B_{2}\right) \mapsto\left(-B_{1}-\zeta \operatorname{id}_{V_{1}}, B_{2}+\zeta \operatorname{id}_{V_{2}}\right)$.

Remark 3.12. This note corresponds to moving parameters of the hyper-Kähler moment map of a moduli space of solutions of Nahm's equations. See also [Tak15, Rem. 2.5].

\subsection{Two-way part}

\subsubsection{Definition}

Let $\mathbf{w} \in \mathbb{Z}_{>0}, \underline{\mathbf{v}} \in \mathbb{Z}_{\geq 0}^{\mathbf{w}+1}$. We consider the following quiver:

$$
V^{0} \underset{C_{1}}{\stackrel{D_{1}}{\leftrightarrows}} V^{1} \stackrel{D_{2}}{\leftrightarrows C_{2}} \cdots \cdots . . . \frac{D_{\mathbf{w}-1}}{\leftrightarrows C_{\mathbf{w}-1}} V^{\mathbf{w}-1} \underset{C_{\mathbf{w}}}{\stackrel{D_{\mathbf{w}}}{\leftrightarrows}} V^{\mathbf{w}}
$$

where $\operatorname{dim} V^{k}=\mathbf{v}_{k}$. Set

$$
\mathbb{M} \equiv \mathbb{M}(\underline{\mathbf{v}} ; \mathbf{w}):=\bigoplus_{k=0}^{\mathbf{w}-1} \operatorname{Hom}\left(V^{k}, V^{k+1}\right) \oplus \operatorname{Hom}\left(V^{k+1}, V^{k}\right) .
$$

It is obvious that $\mathbb{M}$ is a $\sum 2 \mathbf{v}_{k} \mathbf{v}_{k+1}$-dimensional hyper-Kähler manifold. We consider this as a holomorphic symplectic manifold.

$\mathbb{M}$ has a $\mathrm{GL}(\underline{\mathbf{v}})$-hamiltonian action, and its moment map is $\mu_{\mathbf{v}_{k}}=C_{k} D_{k}-D_{k+1} C_{k+1}$. Thus we have a holomorphic symplectic quotient

$$
\widetilde{\mathcal{M}}(\underline{\mathbf{v}} ; \mathbf{w}):=\bigcap_{k=1}^{\mathbf{w}-1} \mu_{\mathbf{v}_{k}}^{-1}(0) / / \prod_{k=1}^{\mathbf{w}-1} \operatorname{GL}\left(\mathbf{v}_{k}\right) .
$$

And define $\mathcal{M}(\underline{\mathbf{v}} ; \mathbf{w})$ as the quotient stack $\left[\widetilde{\mathcal{M}}(\underline{\mathbf{v}} ; \mathbf{w}) / \mathrm{GL}\left(\mathbf{v}_{0}\right) \times \mathrm{GL}\left(\mathbf{v}_{\mathbf{w}}\right)\right]$. Remark that we do not take quotients by the groups at both ends $k=0, \mathbf{w}$ for $\widetilde{\mathcal{M}}(\underline{\mathbf{v}} ; \mathbf{w})$. As a special case, we often consider $\mathbf{v} \cdot \underline{1}:=(\mathbf{v}, \cdots, \mathbf{v})$. The coordinate ring of $\widetilde{\mathcal{M}}(\underline{\mathbf{v}} ; \mathbf{w})$ is generated by entries of 
$C_{\mathbf{w} \cdots 1}:=C_{\mathbf{w}} \cdots C_{1}, D_{1 \cdots \mathbf{w}}:=D_{1} \cdots D_{\mathbf{w}}$ and $D_{1} C_{1}, C_{\mathbf{w}} D_{\mathbf{w}}$. (See e.g. [LBP90]). Therefore we often suppress the two-way parts as

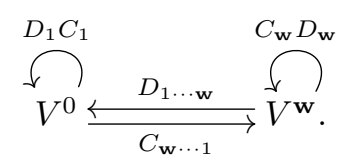

\subsubsection{Dimension of $\widetilde{\mathcal{M}}(\mathbf{v} \cdot \underline{1} ; \mathbf{w})$}

From now on we only consider the case $\underline{\mathbf{v}}=\mathbf{v} \cdot \underline{1}$.

Proposition 3.13. $\bigcap_{k=1}^{\mathbf{w}-1} \mu_{\mathbf{v}_{k}}^{-1}(0)$ has dimension $(\mathbf{w}+1) \mathbf{v}^{2}$.

Proof. We use the flatness criterion in [CB01, Th. 1]. Since we do not take the quotient by the left most and the right most $\mathrm{GL}(V)$, we replace the quiver by a framed quiver of type $A_{\mathbf{w}-1}$ with dimension vectors $\underline{\mathbf{v}}^{\prime}=(\mathbf{v}, \cdots, \mathbf{v}), \underline{\mathbf{w}}^{\prime}=(\mathbf{v}, 0, \cdots, 0, \mathbf{v})$. The criterion in [CB01, Th. 1] for a framed quiver is given in [Nak09, Th. 2.15]. (More precisely, we replace $>$ by $\geq$, as the criterion in [CB01, Th. 2] is given there.) It is easy to check that the criterion is satisfied in this case.

\subsubsection{Factorization}

Let $\Psi$ be a map from $\bigcap \mu_{\mathbf{v}_{k}}^{-1}(0)$ to eigenvalues of $D_{1} C_{1}$ :

$$
\Psi: \bigcap_{k=1}^{\mathbf{w}-1} \mu_{\mathbf{v}_{k}}^{-1}(0) \rightarrow \mathbb{A}^{\mathbf{v}} .
$$

Notice that we can choose any $D_{k} C_{k}$ to define $\Psi$ because the characteristic polynomials for $D_{k} C_{k}$ are all same. For $\mathbf{v}=\mathbf{v}^{\prime}+\mathbf{v}^{\prime \prime}$, take $\left(\mathbb{A}^{\mathbf{v}^{\prime}} \times \mathbb{A}^{\mathbf{v}^{\prime \prime}}\right)_{\text {disj }} \subset \mathbb{A}^{\mathbf{v}^{\prime}} \times \mathbb{A}^{\mathbf{v}^{\prime \prime}}$.

Proposition 3.14. $\mathcal{M}(\mathbf{v} \cdot \underline{1} ; \mathbf{w})$ has a factorization morphism:

$\mathfrak{f}_{\mathbf{v}^{\prime}, \mathbf{v}^{\prime \prime}}: \mathcal{M}(\mathbf{v} \cdot \underline{1} ; \mathbf{w}) \times_{\mathbb{A}}\left(\mathbb{A}^{\mathbf{v}^{\prime}} \times \mathbb{A}^{\mathbf{v}^{\prime \prime}}\right) \operatorname{disj} \stackrel{\sim}{\longrightarrow}\left(\mathcal{M}\left(\mathbf{v}^{\prime} \cdot \underline{1} ; \mathbf{w}\right) \times \mathcal{M}\left(\mathbf{v}^{\prime \prime} \cdot \underline{1} ; \mathbf{w}\right)\right) \times{ }_{\mathbb{v ^ { \prime }}} \times \mathbb{A}^{\mathbf{v}^{\prime \prime}}\left(\mathbb{A}^{\mathbf{v}^{\prime}} \times \mathbb{A}^{\mathbf{v}^{\prime \prime}}\right) \operatorname{disj}$.

Proof. Put $X_{k}=D_{k} C_{k}$ for $1 \leq k \leq \mathbf{w}$ and $X_{\mathbf{w}+1}=C_{\mathbf{w}} D_{\mathbf{w}}$. Let us decompose $V_{k}=V_{k}^{(1)} \oplus V_{k}^{(2)}$, where $V_{k}^{(1)}$ is the eigenspace of $X_{k+1}$ with eigenvalue $w \in \mathbb{C}$, and $V_{k}^{(2)}$ is the sum of the eigenspaces with different eigenvalues. We take a representative

$$
\left(X_{k}, C_{k}, D_{k}\right)=\left(\left[\begin{array}{cc}
X_{k}^{(1)} & 0 \\
0 & X_{k}^{(2)}
\end{array}\right],\left[\begin{array}{cc}
C_{k}^{(11)} & C_{k}^{(12)} \\
C_{k}^{(21)} & C_{k}^{(22)}
\end{array}\right],\left[\begin{array}{cc}
D_{k}^{(11)} & D_{k}^{(12)} \\
D_{k}^{(21)} & D_{k}^{(22)}
\end{array}\right]\right)
$$

And the equations $X_{k+1} C_{k}=C_{k} D_{k} C_{k}=C_{k} X_{k}$ and $X_{k} D_{k}=D_{k} C_{k} D_{k}=D_{k} X_{k+1}$ mean

$$
X_{k+1}^{(1)} C_{k}^{(12)}=C_{k}^{(12)} X_{k}^{(2)}, \quad X_{k}^{(1)} D_{k}^{(12)}=D_{k}^{(12)} X_{k+1}^{(2)} .
$$

Like in the proof of Proposition 3.6, an equation $\left(X_{k+1}^{(1)}-w \text { id }\right)^{l} C_{k}^{(12)}=C_{k}^{(12)}\left(X_{k}^{(2)}-w \text { id }\right)^{l}$ implies $C_{k}^{(12)}=0$. Thus we can conclude that the off-diagonal components of $C_{k}$ and $D_{k}$ vanish. This leads to an isomorphism of stacks from the right hand side to the left hand side.

\subsubsection{Deformation and resolution}

For $\nu^{\mathbb{C}} \in \mathbb{C}^{\mathbf{w}-1}$, we can naturally define a deformation:

$$
\widetilde{\mathcal{M}}_{\nu^{\mathbb{C}}}(\mathbf{v} \cdot \underline{1} ; \mathbf{w}):=\bigcap_{k=1}^{\mathbf{w}-1} \mu_{\mathbf{v}_{k}}^{-1}\left(\nu_{k}^{\mathbb{C}} \mathrm{id}\right) / / \prod_{k=1}^{\mathbf{w}-1} \mathrm{GL}\left(\mathbf{v}_{k}\right) .
$$


Since $\mu_{\mathbf{v}_{k}}$ is flat, this deformation forms a $(\mathbf{w}-1)$-dimensional family. For a deformed two-way part, the argument in the proof of Proposition 3.14 is also valid, so a deformed two-way part has a factorization morphism:

$\mathcal{M}_{\nu^{\mathbb{C}}}(\mathbf{v} \cdot \underline{1} ; \mathbf{w}) \times_{\mathbb{A}^{\mathbf{v}}}\left(\mathbb{A}^{\mathbf{v}^{\prime}} \times \mathbb{A}^{\mathbf{v}^{\prime \prime}}\right)_{\operatorname{disj}} \stackrel{\sim}{\longrightarrow}\left(\mathcal{M}_{\nu \mathbb{C}}\left(\mathbf{v}^{\prime} \cdot \underline{1} ; \mathbf{w}\right) \times \mathcal{M}_{\nu \mathbb{C}}\left(\mathbf{v}^{\prime \prime} \cdot \underline{1} ; \mathbf{w}\right)\right) \times_{\mathbb{A}^{\prime} \times \mathbb{A}^{\prime \prime}}\left(\mathbb{A}^{\mathbf{v}^{\prime}} \times \mathbb{A}^{\mathbf{v}^{\prime \prime}}\right) \operatorname{disj}$

The factorization is compatible also with the stability parameter $\nu^{\mathbb{R}} \in \mathbb{R}^{\mathbf{w}-1}$ : A subspace $S$ or $T$ is invariant under $C, D$, hence also under $C D, D C$. Therefore $S, T$ decompose according to the decomposition $V_{k}=V_{K}^{(1)} \oplus V_{k}^{(2)}$.

\subsection{Proof of Theorem 2.1}

We divide bow solutions into two parts, (1) solutions of Nahm's equation possibly with $I, J$ on an interval containing $\times$, and $(2) B^{L R}, B^{R L}$. For (1) we separate the interval $\left[x_{\sigma, L}, x_{\sigma, R}\right]$ at the middle of segments $\left(x_{\sigma}^{i}, x_{\sigma}^{i+1}\right)$. Correspondingly we take the quotient by the gauge equivalence in two steps. We first take the quotient by gauge transformations which are identity at the end points of intervals and middle points of segments. Then we next take the quotient by the residual group, that is $\prod \mathrm{U}\left(V_{\zeta}\right)$. Thus $\mathcal{M}_{\text {bow }}$ is a hyper-Kähler quotient of the product of the space of solutions of Nahm's equation possibly plus $I, J$, and the quaternionic vector space consisting of (2) by the group $\prod \mathrm{U}\left(V_{\zeta}\right)$.

Now the space of solutions of Nahm's equation possibly plus $I, J$ is described by a triple $(A, a, b)$ together with $B$ at $V_{j}^{w_{j}}$ and $V_{j+1}^{0}$ by Proposition 3.2. For (2) we just set $C=B^{L R}$, $D=B^{R L}$. Those form a quaternionic vector space. Therefore the quotient by the first gauge equivalence is isomorphic to $\widetilde{\mathcal{M}}_{\text {sym }}$. Hence $\mathcal{M}_{\text {bow }}$ is a hyper-Kähler quotient of $\widetilde{\mathcal{M}}_{\text {sym }}$ by $\prod \mathrm{U}\left(V_{\zeta}\right)$, while $\mathcal{M}_{\text {quiver }}$ is a holomorphic symplectic quotient of $\widetilde{\mathcal{M}}_{\text {sym }}$ by $\prod \operatorname{GL}\left(V_{\zeta}\right)$.

The remaining part is a standard identification of GIT quotients and (real) symplectic quotients due to Kempf-Ness, Kirwan and others, as $\widetilde{\mathcal{M}}_{\text {sym }}$ is finite dimensional. Although we do not have an explicit (finite-dimensional) description of the hyper-Kähler metric on $\widetilde{\mathcal{M}}_{\text {sym }}$, the result remains true, as is explained in detail in [Tak15, Prop. 4.7] in a closely related situation.

\section{Basic properties}

\subsection{Stratification}

Let us consider a bow diagram with no triangle part. Then the corresponding bow variety is a quiver variety of affine type $A_{\ell-1}$ without framing (i.e., $W=0$ ) considered in [Nak94b]. Let us denote it by $\mathfrak{M}(\underline{\mathbf{v}})$ to emphasize the dimension vector $\underline{\mathbf{v}}$. Let $\mathfrak{M}^{\mathrm{s}}(\underline{\mathbf{v}})$ be its open subvariety of $\nu^{\mathbb{R}}$-stable points.

We return back to a general bow diagram.

Lemma 4.1. Suppose that a bow diagram contains at least one two-way part, and let $x \in \mathcal{M}$. Then $x$ has a representative $(A, B, a, b, C, D) \in \widetilde{\mathcal{M}}$ which is a direct sum

$$
\left(A^{s}, B^{s}, a^{s}, b^{s}, C^{s}, D^{s}\right) \oplus \bigoplus_{k}\left(A^{k}=\mathrm{id}, B^{k}, 0,0, C^{k}, D^{k}\right)
$$

such that

1. $\left(A^{s}, B^{s}, a^{s}, b^{s}, C^{s}, D^{s}\right)$ is $\nu^{\mathbb{R}}$-stable.

2. $\left(C^{k}, D^{k}\right)$ represents a point in $\mathfrak{M}^{\mathrm{s}}\left(\underline{\mathbf{v}}^{k}\right)$, and is extended to a bow by setting $A^{k}=\mathrm{id}, B^{k}$ is an appropriate $C^{k} D^{k}$ or $D^{k} C^{k}$.

Note that $\mathfrak{M}^{\mathrm{s}}\left(\underline{\mathbf{v}}^{k}\right)$ is nonempty only if $\nu \cdot \underline{\mathbf{v}}^{k}=\left(\nu^{\mathbb{R}} \cdot \underline{\mathbf{v}}^{k}, \nu^{\mathbb{C}} \cdot \underline{\mathbf{v}}^{k}\right)=0$. 
Proof. We take $(A, B, a, b, C, D) \in \widetilde{\mathcal{M}}^{\text {ss }}$ so that it has a closed $\mathcal{G}=\prod \mathrm{GL}\left(V_{\zeta}\right)$-orbit in $\widetilde{\mathcal{M}}^{\text {ss }}$.

If it is not $\nu^{\mathbb{R}}$-stable, there is a collection of subspaces $S_{\zeta}$ or $T_{\zeta}$ as in $(\nu 1),(\nu 2)$, where inequalities are equalities. As in the proof of Proposition 2.8, we take a 1-parameter subgroup $\rho(t)$ so that the limit is the associated graded. But the limit stays in the same orbit as we assume $(A, B, a, b, C, D)$ has a closed orbit. Therefore $(A, B, a, b, C, D)$ is a direct sum of $\nu^{\mathbb{R}_{-}}$ stable $\left(A^{s}, B^{s}, a^{s}, b^{s}, C^{s}, D^{s}\right)$ and the data for $S=\left\{S_{\zeta}\right\}$ or $V / T=\left\{V_{\zeta} / T_{\zeta}\right\}$. We also see that $V=V / T \oplus T$, hence we do not need to consider the case $V / T$.

Let us consider the second summand. By the conditions in $(\nu 1),(\nu 2)$, the induced $a, b$ are zero on $S$ or $V / T$, and $A$ is an isomorphism. So we can set $A=$ id. From the equation $B A=A B$, all $B$ on a wavy line are equal. Hence it is enough to determine either end of the wavy line, but it is given by an appropriate $C D$ or $D C$. Thus the second summand can be regarded as a point in $\mathfrak{M}(\underline{\mathbf{v}})$ with the dimension vector $\underline{\mathbf{v}}$ given by $\left(\operatorname{dim} S_{\sigma}\right)$.

The induced $C, D$ on $S$ is not necessary $\nu^{\mathbb{R}}$-stable, but it decomposes into a direct sum of $\nu^{\mathbb{R}}$-stable as it has a closed orbit.

The case when there is no two-way part can be treated in the same way, but let us postpone it till Proposition 6.5.

Remark 4.2. A description of $\mathfrak{M}^{\mathrm{s}}\left(\underline{\mathbf{v}}^{k}\right)$ is given in [Nak09, Lem. 3.2]. There are two types:

1. $\underline{\mathbf{v}}^{k}=\delta$, the primitive positive imaginary root, and $\mathfrak{M}^{\mathrm{s}}(\delta)$ is the regular locus of a partial resolution of the deformation of $\mathbb{C}^{2} /(\mathbb{Z} / \ell \mathbb{Z})$ introduced in [Kro89] as a hyper-Kähler quotient. Note also $\delta=(1,1, \ldots, 1)$ since we are considering type $A$.

2. $\underline{\mathbf{v}}^{k}$ is a real positive root, indecomposable among roots in $\nu^{\perp}$, and $\mathfrak{M}^{\mathrm{s}}\left(\underline{\mathbf{v}}^{k}\right)$ is a single point.

The proof is given by the reduction to Crawley-Boevey's criterion [CB01] and the hyper-Kähler rotation, hence under a restriction on $\nu$. Since Crawley-Boevey's criterion is generalized in [BS16], the same results remain true for any $\nu$.

Let us consider two extreme cases (a) $\nu$ is not contained in any root hyperplanes, (b) $\nu=0$. In case (a), $\mathfrak{M}^{\mathrm{s}}(\underline{\mathbf{v}})$ is empty, hence we have $\mathcal{M}=\mathcal{M}^{\mathrm{s}}$. In case (b), the first type of $\mathfrak{M}^{\mathrm{s}}(\delta)$ is just $\mathbb{C}^{2} \backslash\{0\} /(\mathbb{Z} / \ell \mathbb{Z})(\ell \neq 1)$ or $\mathbb{C}^{2}(\ell=1)$, and the second type means that $\underline{\mathbf{v}}^{k}$ is a coordinate vector.

This lemma together with the above remark gives us a stratification of $\mathcal{M}$. Let us specify the dimension vector $\mathbf{v}$ in the notation of a bow variety as $\mathcal{M}(\underline{\mathbf{v}})$. The underlying graph, $\mathrm{O}$ and $\times$ on the circle, is fixed, but we will vary dimension vectors. We will use a similar looking notation $\mathcal{M}(\underline{\mathbf{v}}, \underline{\mathbf{w}})$ in $\S 6.1$, where the underlying graph is determined by $\underline{\mathbf{v}}, \underline{\mathbf{w}}$. There are no relations among them.

Let us suppose $\nu \cdot \delta=0$ so that the first type of $\mathfrak{M}^{\mathrm{s}}(\delta)$ actually occurs. (The case $\nu \cdot \delta \neq 0$ has a simpler stratification.) The stratification is

$$
\mathcal{M}(\underline{\mathbf{v}})=\bigsqcup \mathcal{M}^{\mathrm{s}}\left(\underline{\mathbf{v}}^{\prime}\right) \times S^{\underline{k}}\left(\mathfrak{M}^{\mathrm{s}}(\delta)\right),
$$

where the summation runs over $\underline{\mathbf{v}}^{\prime}$ with $\mathcal{M}^{\mathrm{s}}\left(\underline{\mathbf{v}}^{\prime}\right) \neq \emptyset$ and a partition $\underline{k}$ such that $\underline{\mathbf{v}}-\underline{\mathbf{v}}^{\prime}$ $|\underline{k}|(1,1, \ldots, 1)$ is in $\nu^{\perp}$ and has nonnegative entries. Here $|\underline{k}|$ is the weight of $\underline{k}$, and $S^{\underline{k}}\left(\mathfrak{M}^{\mathrm{s}}(\delta)\right)$ is the stratum of the symmetric product $S^{|\underline{k}|}\left(\mathfrak{M}^{\mathrm{s}}(\delta)\right)$ consisting of configurations whose multiplicities are given by $\underline{k}$. We do not write the second type $\mathfrak{M}^{\mathrm{s}}\left(\underline{\mathbf{v}}^{k}\right)$ explicitly as it is a point.

This is a general result, but a little imprecise as we do not have a criterion for $\mathcal{M}^{\mathrm{s}}\left(\underline{\mathbf{v}}^{\prime}\right) \neq \emptyset$ at this moment. When the cobalanced condition is satisfied for $\mathbf{v}$, it is also satisfied for $\mathbf{v}^{\prime}$, thanks to the above description of $\underline{\mathbf{v}}^{k}$. In fact, the above coincides with the stratification as a quiver variety [Nak94b, §6], [Nak98, §3(v)]. Hence we have the criterion for $\mathcal{M}^{\mathrm{s}}\left(\underline{\mathbf{v}}^{\prime}\right) \neq \emptyset[\mathrm{CB} 01]$. On the other hand, the balanced condition for $\mathbf{v}$ is not inherited to $\underline{\mathbf{v}}^{\prime}$. Nevertheless we will determine the stratification for bow varieties with balanced condition in $\S \S 7.5,7.7$. 


\subsection{Slice to a stratum}

Let us continue study of the stratification (4.3). As in the case of quiver varieties ([Nak01, §3]), we can study the local (in the complex analytic topology) structure of $\mathcal{M}$ around a point in a stratum by using the local normal form of the moment map. The result used there ([Nak01, Lem. 3.2.1]) is general, and applicable to the current situation. (See also [Los06, Th. 3].)

We use the description of $\mathcal{M}$ as a symplectic reduction of $\widetilde{\mathcal{M}}_{\text {sym }}$ by $\mathcal{G}$ as in the proof of Proposition 2.13. We take $x \in \mathcal{M}$ and its representative $m$ in $\widetilde{\mathcal{M}}_{\text {sym }}$ so that it has a closed orbit in $\widetilde{\mathcal{M}}^{\text {ss }}$. Let $\widehat{\mathcal{G}}$ be the stabilizer of $m$. Let $\mathcal{G} m$ denote the orbit through $m$, and $T_{m} \mathcal{G} m$ its tangent space at $m$. The latter is a subspace of $T_{m} \widetilde{\mathcal{M}}_{\text {sym }}$ the tangent space of $\mathcal{\mathcal { M }}_{\text {sym }}$ at $m$. We consider the symplectic normal $\widehat{\mathbf{M}}$ defined as $\left(T_{m} \mathcal{G} m\right)^{\perp} / T_{m} \mathcal{G} m$, where $\left(T_{m} \mathcal{G} m\right)^{\perp}$ is the symplectic perpendicular of $T_{m} \mathcal{G} m$ in $T_{m} \widetilde{\mathcal{M}}_{\text {sym }}$. The stabilizer $\widehat{\mathcal{G}}$ naturally acts on $\widehat{\mathbf{M}}$. Let $\widehat{\mu}: \widehat{\mathbf{M}} \rightarrow \widehat{\mathfrak{g}}^{*}$ be the moment map vanishing at the origin. Here $\widehat{\mathfrak{g}}$ is the Lie algebra of $\widehat{\mathcal{G}}$. Then a general result mentioned above says that there is a local symplectic isomorphism

$$
(\mathcal{M}, x) \cong\left(\widehat{\mu}^{-1}(0) / / \widehat{\mathcal{G}}, 0\right) .
$$

Let us apply this result to our situation. Let $x \in \mathcal{M}$ and take a representative $m$. The tangent space $T_{m} \widetilde{\mathcal{M}}_{\text {sym }}$ is $\operatorname{Ker} \beta_{1}$ in (2.11). Since $\alpha$ is the differential of the $\mathcal{G}$-action, the tangent space $T_{m} \mathcal{G} m$ of the orbit is $\operatorname{Im} \alpha$. Since $\beta_{2}$ is the differential of $\mu_{2}$, which is the moment map of $\widetilde{\mathcal{M}}_{\text {sym }}$ for the $\mathcal{G}$-action, the symplectic perpendicular $\left(T_{m} \mathcal{G} m\right)^{\perp}$ is $\operatorname{Ker} \beta_{2}$. Hence

$$
\widehat{\mathbf{M}} \cong \operatorname{Ker} \beta / \operatorname{Im} \alpha \text {. }
$$

Let us take the representative $m$ as in Lemma 4.1. We collect isomorphic $\left(A^{k}, B^{k}, 0,0, C^{k}, D^{k}\right)$ and write the second summand as

$$
\bigoplus_{k}\left(A^{k}, B^{k}, 0,0, C^{k}, D^{k}\right)^{\oplus n_{k}}
$$

with the multiplicity $n_{k} \in \mathbb{Z}_{>0}$. The stabilizer $\widehat{\mathcal{G}}$ of $m$ is $\prod \operatorname{GL}\left(n_{k}\right)$. In fact, there is no nonzero homomorphism between different summand thanks to the stability condition. Let us formally add $k=\infty$ to the index set corresponding to the first summand in Lemma 4.1. We set $n_{\infty}=1$. (Note however that the stabilizer $\widehat{\mathcal{G}}$ does not contain the factor $\operatorname{GL}\left(n_{\infty}\right)$.) Let us denote by $V_{\zeta}^{k}$ the vector space for a segment $\zeta$ corresponding to the direct summand $\left(A^{k}, B^{k}, 0,0, C^{k}, D^{k}\right)$ and also for $k=\infty$.

We have the corresponding decomposition of $\operatorname{Ker} \beta / \operatorname{Im} \alpha$ as

$$
\bigoplus_{k, l} \operatorname{Hom}\left(\mathbb{C}^{n_{k}}, \mathbb{C}^{n_{l}}\right) \otimes E^{k l}
$$

where $E^{k l}$ is the middle cohomology of the three term complex

$$
\begin{array}{r}
\bigoplus_{\zeta} \operatorname{Hom}\left(V_{\zeta}^{k}, V_{\zeta}^{l}\right) \stackrel{\alpha^{k l}}{\longrightarrow} \mathbb{M}^{k l} \stackrel{\beta^{k l}}{\longrightarrow} \mathbb{N}^{k l}:=\bigoplus_{x} \operatorname{Hom}\left(V_{\zeta_{x}^{-}}^{k}, V_{\zeta_{x}^{+}}^{l}\right) \oplus \bigoplus_{\zeta} \operatorname{Hom}\left(V_{\zeta}^{k}, V_{\zeta}^{l}\right), \\
\mathbb{M}^{k l}:=\bigoplus_{x}\left(\operatorname{Hom}\left(V_{\zeta_{x}^{-}}^{k}, V_{\zeta_{x}^{-}}^{l}\right) \oplus \operatorname{Hom}\left(V_{\zeta_{x}^{+}}^{k}, V_{\zeta_{x}^{+}}^{l}\right) \oplus \operatorname{Hom}\left(V_{\zeta_{x}^{-}}^{k}, V_{\zeta_{x}^{+}}^{l}\right) \oplus \operatorname{Hom}\left(\delta_{l \infty} \mathbb{C}_{x}, V_{\zeta_{x}^{+}}^{l}\right)\right. \\
\left.\oplus \operatorname{Hom}\left(V_{\zeta_{x}^{-}}^{k}, \delta_{k \infty} \mathbb{C}_{x}\right)\right) \oplus \bigoplus_{h}\left(\operatorname{Hom}\left(V_{\mathrm{o}(h)}^{k}, V_{\mathrm{i}(h)}^{l}\right) \oplus \operatorname{Hom}\left(V_{\mathrm{i}(h)}^{k}, V_{\mathrm{o}(h)}^{l}\right)\right) .
\end{array}
$$

Here $\delta_{k \infty} \mathbb{C}_{x}, \delta_{l \infty} \mathbb{C}_{x}$ mean that we set them as 0 unless $k=\infty$ or $l=\infty$. The definition of $\alpha^{k l}$, $\beta^{k l}$ are as in (2.11), we replace $A, B, \ldots$ with appropriate $A^{k}, A^{l}, B^{k}, B^{l}, \ldots$ The action of the stabilizer $\widehat{\mathcal{G}}=\prod \operatorname{GL}\left(n_{k}\right)$ is the standard one on $\operatorname{Hom}\left(\mathbb{C}^{n_{k}}, \mathbb{C}^{n_{l}}\right)$ with the trivial one on $E^{k l}$.

The symplectic reduction $\widehat{\mu}^{-1}(0) / / \widehat{\mathcal{G}}$ is a product of a quiver variety $\mathfrak{M}_{0}$ and an affine space as follows. We define a quiver by 
- the set of vertices is $\{k\} \backslash\{\infty\}$,

- the number of arrows from $k$ to $l$ is $2 \delta_{k l}-{ }^{t} \underline{\mathbf{v}}^{k} C \underline{\mathbf{v}}^{l}$.

Then we put two vector spaces for each vertex $k$ :

- $\mathbb{C}^{n_{k}}$ for the quiver part,

- $E^{k \infty}$ for the framing.

The affine space is the additional factor $\operatorname{Hom}\left(\mathbb{C}^{n_{\infty}}, \mathbb{C}^{n_{\infty}}\right) \otimes E^{\infty \infty}=E^{\infty \infty}$ for $k=l=\infty$, where $\widehat{\mathcal{G}}$ acts trivially. It corresponds to the tangent space of $\mathcal{M}^{\mathrm{s}}\left(\underline{\mathbf{v}}^{\prime}\right)$ for the first factor in (4.3).

The number $2 \delta_{k l}-{ }^{t} \underline{\mathbf{v}}^{k} C \underline{\mathbf{v}}^{l}$ is given by computing $\operatorname{dim} E^{k l}$ : By the stability as mentioned above, we have $\operatorname{dim} \operatorname{Ker} \alpha^{k l}=\delta_{k l}$. The first component of $\beta^{k l}$ is surjective by Proposition 2.12(2). Since the second component of $\beta^{k l}$ is coming from the differential of the moment map, we have $\operatorname{dim}$ Coker $\beta^{k l}=\delta_{k l}$. Therefore $\operatorname{dim} E^{k l}=\operatorname{dim} \mathbb{M}^{k l}-\sum_{\zeta} \operatorname{dim} \operatorname{Hom}\left(V_{\zeta}^{k}, V_{\zeta}^{l}\right)-\operatorname{dim} \mathbb{N}^{k l}+2 \delta_{k l}$. We further observe that contributions from triangular parts cancel for $k, l \neq \infty$. Therefore $\operatorname{dim} E^{k l}=2 \delta_{k l}-{ }^{t}{ }^{k} C \underline{\mathbf{v}}^{l}$ as in the case of quiver varieties. In particular, the underlying quiver is a union of Jordan quivers corresponding to $\underline{\mathbf{v}}^{k}=\delta$, and an affine quiver of type $A$ corresponding to other $\underline{\mathbf{v}}^{k}$.

For $k=l \neq \infty$, the action of $\widehat{\mathcal{G}}$ on the subspace $\mathbb{C}$ id $\otimes E^{k l} \subset \operatorname{Hom}\left(\mathbb{C}^{n_{k}}, \mathbb{C}^{n_{k}}\right) \otimes E^{k k}$ is trivial. It is the tangent space of $\mathcal{M}\left(\underline{\mathbf{v}}^{k}\right)$, which is non trivial only when $\underline{\mathbf{v}}^{k}=\delta$. But this part is contained in the quiver variety part. When we compute the transversal slice, this factor must be factored out. See Theorem 7.26.

Proposition 4.4. We have a local isomorphism

$$
(\mathcal{M}, x) \cong\left(\mathfrak{M}_{0} \times E^{\infty \infty},(0,0)\right)
$$

in complex analytic topology.

\subsection{Semismall morphism}

By Remark 4.2, the space $\left(\sqrt{-1} \mathbb{R}^{3}\right)^{\mathcal{I}}$ of parameter $\nu$ have a collection of codimension 3 subspaces defined by roots of the affine Lie algebra of type $A_{\ell-1}$. If a dimension vector is fixed, only finitely many roots contribute as $\underline{\mathbf{v}}^{k}$ cannot have greater entries. We thus have a face structure on $\left(\sqrt{-1} \mathbb{R}^{3}\right)^{\mathcal{I}}$. See $[$ Nak09, $\S 2.3]$ for detail.

We choose $\nu^{\bullet}$ which is contained in a subspace, and $\nu$ so that 1) it has the same complex part $\left.\nu^{\bullet}, \mathbb{C}=\nu^{\mathbb{C}}, 2\right)$ it is not contained in any of subspaces, and 3) $\nu^{\bullet, \mathbb{R}}$ is contained in the closure of the chamber containing $\nu^{\mathbb{R}}$. The most important example is $\nu^{\bullet}=0, \nu=\left(\nu^{\mathbb{R}}, 0\right)$ with $\nu^{\mathbb{R}}$ not contained in root hyperplanes.

Under the assumption the $\nu^{\mathbb{R}}$-stability implies $\nu^{\bullet}, \mathbb{R}_{\text {-semistability. We have the induced mor- }}$ phism $\pi: \mathcal{M}_{\nu} \rightarrow \mathcal{M}_{\nu} \bullet$. The local description in a neighborhood of $m \in \mathcal{M}_{\nu} \bullet$ can be lifted to $\mathcal{M}_{\nu}$ : there are neighborhood $U$ and $V$ of $m$ and 0 in $\mathcal{M}_{\nu} \bullet$ and $\mathfrak{M}_{0}$ respectively such that there is a commutative diagram

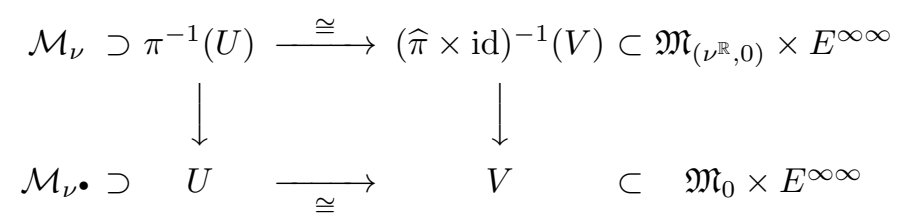

See [Nak01, §3], [Nak09, §2.7]. Here $\nu^{\mathbb{R}}$ is regarded as a character of $\widehat{\mathcal{G}}$ by restriction. In particular,

Proposition 4.5. The image $\pi\left(\mathcal{M}_{\nu}\right)$ is a union of strata in (4.3). If we replace $\mathcal{M}_{\nu} \bullet$ by the image $\pi\left(\mathcal{M}_{\nu}\right), \pi$ is semismall with respect to the induced stratification. Moreover all strata are relevant, i.e., the dimension of the fiber is the half of codimenion of a stratum. 
See [Nak09, Rem. 2.24] for the last statement. It also follows that the union of smaller strata are the singular points of $\pi\left(\mathcal{M}_{\nu}\right)$. In fact, if a point $x$ in a smaller stratum is a regular point, the symplectic form extends across $x$, as the codimension of the stratum is greater than or equal to 2 . But as $\pi$ respect symplectic forms on regular loci, $\pi$ becomes an isomorphism at $x$. But $\pi^{-1}(x)$ cannot be a point, as the stratum is relevant.

In particular, we see that (4.3) is the decomposition into symplectic leaves.

Remark 4.6. Since $\pi$ is semismall, $\pi_{*}\left(\mathbb{C}_{\mathcal{M}_{\nu}}\left[\operatorname{dim} \mathcal{M}_{\nu}\right]\right)$ is a semisimple perverse sheaf. It is probably true that an intersection cohomology complex with nontrivial local coefficients does not appear in the summand as for the case of quiver varieties. It should be possible to prove it by an argument in [Nak09, a paragraph after Rem. 5.13]. If this is true, the multiplicities of IC sheaves in $\pi_{*}\left(\mathbb{C}_{\mathcal{M}_{\nu}}\left[\operatorname{dim} \mathcal{M}_{\nu}\right]\right)$ are dimensions of top degree homology groups of fibers, and hence are equal to the corresponding multiplicities for quiver varieties. They are computed in [Nak09].

\section{Symplectic structure}

We study the symplectic structure on bow varieties in this section.

The symplectic structure on the two-way part is $\operatorname{tr}(d C \wedge d D)$. The Poisson bracket is

$$
\left\{C_{i j}, D_{k l}\right\}=-\delta_{i l} \delta_{j k}
$$

\subsection{Poisson structure on the triangle part}

Let us review the Poisson structure defined on the 'triangle' part $\widetilde{\mathcal{M}}$ in $\S 3.1$ in [FR14].

Let $\mathfrak{n}\left(\mathbf{v}_{1}, \mathbf{v}_{2}\right):=V_{1} \oplus V_{2}^{*} \oplus \operatorname{Hom}\left(V_{2}, V_{1}\right)$. We equip a Lie algebra structure on $\mathfrak{n}\left(V_{1}, V_{2}\right)$ so that $\operatorname{Hom}\left(V_{2}, V_{1}\right)$ is central, $\left[V_{1}, V_{1}\right]=0=\left[V_{2}^{*}, V_{2}^{*}\right]$ and $\left[v, w^{\vee}\right]=v \otimes w^{\vee}$ for $v \in V_{1}, w^{\vee} \in V_{2}^{*}$.

We have a natural action of $\mathfrak{g l}\left(\mathbf{v}_{1}\right) \oplus \mathfrak{g l}\left(\mathbf{v}_{2}\right)$ on $\mathfrak{n}\left(V_{1}, V_{2}\right)$ induced from the tautological actions of $\mathfrak{g l}\left(\mathbf{v}_{1}\right)$ on $V_{1}$ and $\mathfrak{g l}\left(\mathbf{v}_{2}\right)$ on $V_{2}$. It acts by derivations. We consider the semi-direct product

$$
\mathfrak{a}:=\left(\mathfrak{g l}\left(\mathbf{v}_{1}\right) \oplus \mathfrak{g l}\left(\mathbf{v}_{2}\right)\right) \rtimes \mathfrak{n}\left(\mathbf{v}_{1}, \mathbf{v}_{2}\right)
$$

We give a structure of a Poisson manifold on $\mathfrak{a}^{*}$ by $\{X, Y\}=[X, Y]$ for $X, Y \in \mathfrak{a}$.

We have a natural action of $\operatorname{GL}\left(\mathbf{v}_{1}\right) \times \mathrm{GL}\left(\mathbf{v}_{2}\right)$ on $\mathfrak{a}^{*}$, which preserves the Poisson structure. Recall a $\mathfrak{g l}\left(\mathbf{v}_{1}\right)^{*} \oplus \mathfrak{g l}\left(\mathbf{v}_{2}\right)^{*}$-valued map $m$ on the Poisson manifold $\mathfrak{a}^{*}$ with the $\mathrm{GL}\left(\mathbf{v}_{1}\right) \times \operatorname{GL}\left(\mathbf{v}_{2}\right)$ action is a moment map if $\{\langle\xi, m\rangle, f\}=\xi^{*} f$ for any function $f$ and $\xi \in \mathfrak{g l}\left(\mathbf{v}_{1}\right) \oplus \mathfrak{g l}\left(\mathbf{v}_{2}\right)$. Here $\xi^{*}$ is the vector field generated by $\xi$ and $\xi^{*} f$ is the differential of $f$ by $\xi^{*}$. By our definition of the Poisson bracket, $m$ in our case is just given by the projection $\mathfrak{a}^{*} \rightarrow \mathfrak{g l}\left(\mathbf{v}_{1}\right)^{*} \oplus \mathfrak{g l}\left(\mathbf{v}_{2}\right)^{*}$.

We write entries of $\mathfrak{a}^{*}=\operatorname{Hom}\left(V_{1}, V_{2}\right) \oplus \mathfrak{g l}\left(\mathbf{v}_{1}\right) \oplus \mathfrak{g l}\left(\mathbf{v}_{2}\right) \oplus V_{2} \oplus V_{1}^{*}$ by $A, B, B^{\prime}, a, b$. This is almost the same as the notation in $\S 3.1$, but we will set $B_{1}=-B, B_{2}=B^{\prime}$ later. Let $A_{i j}$ be the $(i, j)$ matrix unit of $\operatorname{Hom}\left(V_{2}, V_{1}\right)$. We consider it as a linear function on $\operatorname{Hom}\left(V_{1}, V_{2}\right)$ by $A \mapsto \operatorname{tr}\left(A A_{i j}\right)$, and then extend it to $\mathfrak{a}^{*}$. In other words, $A_{i j}$ is the function on $\mathfrak{a}^{*}$ giving by the $(j, i)$ entry of $A$. We introduce $B_{i j}, B_{i j}^{\prime}, a_{i}, b_{j}$ in the same way. Then the Poisson brackets are given by (see [FR14, (11-15)])

$$
\begin{gathered}
\left\{A_{i j}, A_{k l}\right\}=0, \quad\left\{a_{i}, a_{j}\right\}=0=\left\{b_{i}, b_{j}\right\}, \\
\left\{B_{i j}, B_{k l}\right\}=\delta_{j k} B_{i l}-\delta_{l i} B_{k j}, \quad\left\{B_{i j}^{\prime}, B_{k l}^{\prime}\right\}=\delta_{j k} B_{i l}^{\prime}-\delta_{l i} B_{k j}^{\prime}, \\
\left\{B_{i j}, a_{k}\right\}=0=\left\{B_{i j}^{\prime}, b_{k}\right\}, \quad\left\{B_{i j}, B_{k l}^{\prime}\right\}=0, \\
\left\{B_{i j}, b_{k}\right\}=\delta_{j k} b_{i}, \quad\left\{B_{i j}^{\prime}, a_{k}\right\}=-\delta_{k i} a_{j}, \\
\left\{B_{i j}, A_{k l}\right\}=\delta_{j k} A_{i l}, \quad\left\{B_{i j}^{\prime}, A_{k l}\right\}=-\delta_{l i} A_{k j}, \\
\left\{b_{i}, a_{j}\right\}=A_{i j}, \quad\left\{A_{i j}, b_{k}\right\}=0=\left\{A_{i j}, a_{k}\right\} .
\end{gathered}
$$

We set

$$
\mu\left(A, B, B^{\prime}, a, b\right):=B^{\prime} A+A B+a b .
$$


Proposition 5.3 ([FR14, Prop. 3.15]). The ideal generated by entries of $\mu$ is a Poisson ideal.

Proof. Since we have decided to write the $(j, i)$ entry of $A$ by $A_{i j}$, the $(j, i)$ entry $\mu_{i j}$ of $\mu$ is

$$
\mu_{i j}\left(A, B, B^{\prime}, a, b\right)=\sum_{m}\left(B_{m j}^{\prime} A_{i m}+A_{m j} B_{i m}\right)+a_{j} b_{i} .
$$

Hence

$$
\begin{aligned}
\left\{\mu_{i j}, B_{k l}\right\} & =\left\{\sum_{m}\left(B_{m j}^{\prime} A_{i m}+A_{m j} B_{i m}\right)+a_{j} b_{i}, B_{k l}\right\} \\
& =\sum_{m}\left(-B_{m j}^{\prime} \delta_{i l} A_{k m}-\delta_{l m} A_{k j} B_{i m}+A_{m j}\left(\delta_{m k} B_{i l}-\delta_{i l} B_{k m}\right)\right)-a_{j} \delta_{i l} b_{k} \\
& =-\delta_{i l} \mu_{k j} .
\end{aligned}
$$

Computation of other Poisson brackets are similar. We get

$$
\begin{gathered}
\left\{\mu_{i j}, B_{k l}^{\prime}\right\}=\delta_{j k} \mu_{i l}, \quad\left\{\mu_{i j}, A_{k l}\right\}=0, \\
\left\{\mu_{i j}, a_{k}\right\}=0=\left\{\mu_{i j}, b_{k}\right\} .
\end{gathered}
$$

Therefore a subvariety $\mu=0$ has the induced Poisson structure. Setting $B_{1}=-B, B_{2}=B^{\prime}$, we have the Poisson structure on $\widetilde{\mathcal{M}}$. The $\mathrm{GL}\left(\mathbf{v}_{1}\right) \times \mathrm{GL}\left(\mathbf{v}_{2}\right)$-action preserves $\mu=0$, and the moment map is given by the projection to $\mathfrak{g l}\left(\mathbf{v}_{1}\right)^{*} \oplus \mathfrak{g l}\left(\mathbf{v}_{2}\right)^{*}$. Its restriction coincides with the moment map on $\widetilde{\mathcal{M}}$ in $\S 3.1$.

Remark 5.4. The Poisson brackets (5.1) and (5.2) are compatible in bow varieties. To see this, we consider products of two-way and triangle parts, say $\left(\operatorname{Hom}\left(V_{0}, V_{1}\right) \oplus \operatorname{Hom}\left(V_{1}, V_{0}\right)\right) \times \widetilde{\mathcal{M}}$, and check that entries of $B-C D$ are Poisson commuting with functions invariant under the $\mathrm{GL}\left(V_{1}\right)$-action. For example,

$$
\begin{aligned}
& \left\{B_{i j}-\sum_{m} C_{m j} D_{i m}, \sum_{n} A_{n l} C_{k n}\right\} \\
= & \sum_{n}\left\{B_{i j}, A_{n l}\right\} C_{k n}-\sum_{m} C_{m j} A_{n l}\left\{D_{i m}, C_{k n}\right\}=A_{i l} C_{k j}-C_{k j} A_{i l}=0 .
\end{aligned}
$$

\subsection{Poisson structure on the slice}

Let $T^{*} \mathrm{GL}(n) \cong \mathrm{GL}(n) \times \mathfrak{g l}(n)$. It has a symplectic form as a cotangent bundle. We consider the associated Poisson manifold. Let $u_{i j}, \eta_{i j}$ be the $(j, i)$ entries of $u, \eta$ for $(u, \eta) \in \operatorname{GL}(n) \times \mathfrak{g l}(n)$ respectively. We consider them as functions on $\operatorname{GL}(n) \times \mathfrak{g l}(n)$. The Poisson brackets are

$$
\left\{u_{i j}, u_{k l}\right\}=0, \quad\left\{u_{i j}, \eta_{k l}\right\}=\delta_{j k} u_{i l}, \quad\left\{\eta_{i j}, \eta_{k l}\right\}=\delta_{j k} \eta_{i l}-\delta_{l i} \eta_{k j} .
$$

We note

$$
\left\{\eta_{i j}, u^{k l}\right\}=\delta_{j k} u^{i l}
$$

where $u^{k l}$ is the $(l, k)$-entry of $u^{-1}$.

We also consider $V \oplus V^{*}$ as in $\S 3.1$. We write $I$ for $V, J$ for $V^{*}$, and define functions $I_{i}$ and $J_{j}$ on $V, V^{*}$ by taking $i$ th and $j$ th entries respectively. The Poisson brackets are

$$
\left\{I_{i}, I_{j}\right\}=0=\left\{J_{i}, J_{j}\right\}, \quad\left\{I_{i}, J_{j}\right\}=-\delta_{i j} .
$$

Now we consider Poisson brackets on the slice. One can compute them by using Corollary 3.3, and find the same formula as above. But it is obvious once we notice that $\operatorname{GL}(n) \times \mathcal{S}$ is a symplectic reduction of $T^{*} \mathrm{GL}(n)$ as we have explained in Remark 3.4. 


\subsection{Poisson isomorphism for triangle part}

Proposition 5.7. The isomorphism between the space of Hurtubise normal forms and $\widetilde{\mathcal{M}}$ in Proposition 3.2 respects the Poisson structures.

Proof. Let $\Phi$ be the morphism from the space of Hurtubise normal forms to $\widetilde{\mathcal{M}}$.

Suppose $\mathbf{v}_{1}=\mathbf{v}_{2}$. We have $A=u, B=-u^{-1} \eta u, B^{\prime}=\eta-I J, a=I, b=J u$. Note $B_{i j}^{\prime}=\eta_{i j}-I_{j} J_{i}$ in our convention.

Using (5.5), we compute brackets not involving $B$ as

$$
\begin{gathered}
\left\{\Phi^{*} a_{i}, \Phi^{*} a_{j}\right\}=\left\{I_{i}, I_{j}\right\}=0, \quad\left\{\Phi^{*} b_{i}, \Phi^{*} b_{j}\right\}=\sum_{m, n}\left\{J_{m} u_{i m}, J_{n} u_{j n}\right\}=0, \\
\left\{\Phi^{*} A_{i j}, \Phi^{*} A_{k l}\right\}=\left\{u_{i j}, u_{k l}\right\}=0, \\
\left\{\Phi^{*} B_{i j}^{\prime}, \Phi^{*} B_{k l}^{\prime}\right\}=\left\{\eta_{i j}-I_{j} J_{i}, \eta_{k l}-I_{l} J_{k}\right\}=\delta_{j k} \eta_{i l}-\delta_{l i} \eta_{k j}+\delta_{i l} I_{j} J_{k}-\delta_{k j} I_{l} J_{i} \\
=\delta_{j k}\left(\eta_{i l}-I_{l} J_{i}\right)-\delta_{l i}\left(\eta_{k j}-I_{j} J_{k}\right)=\delta_{j k} \Phi^{*} B_{i l}^{\prime}-\delta_{l i} \Phi^{*} B_{k j}^{\prime}, \\
\left\{\Phi^{*} B_{i j}^{\prime}, \Phi^{*} A_{k l}\right\}=\left\{\eta_{i j}-I_{j} J_{i}, u_{k l}\right\}=-\delta_{l i} u_{k j}=-\delta_{l i} \Phi^{*} A_{k j}, \\
\left\{\Phi^{*} b_{i}, \Phi^{*} a_{j}\right\}=\sum_{m}\left\{J_{m} u_{i m}, I_{j}\right\}=u_{i j}=\Phi^{*} A_{i j}, \\
\left\{\Phi^{*} B_{i j}^{\prime}, \Phi^{*} a_{k}\right\}=\left\{\eta_{i j}-I_{j} J_{i}, I_{k}\right\}=-\delta_{i k} I_{j}=-\delta_{i k} \Phi^{*} a_{j}, \\
\left\{\Phi^{*} B_{i j}^{\prime}, \Phi^{*} b_{k}\right\}=\sum_{m}\left\{\eta_{i j}-I_{j} J_{i}, J_{m} u_{k m}\right\}=\sum_{m}\left(-\delta_{m i} u_{k j} J_{m}+\delta_{j m} J_{i} u_{k m}\right)=0, \\
\left\{\Phi^{*} A_{i j}, \Phi^{*} a_{k}\right\}=\left\{u_{i j}, I_{k}\right\}=0, \\
\left\{\Phi^{*} A_{i j}, \Phi^{*} b_{k}\right\}=\sum_{m}\left\{u_{i j}, J_{m} u_{k m}\right\}=0 .
\end{gathered}
$$

These are the same as (5.2). Thus the assertion is checked for these brackets.

To compute brackets with $B_{i j}$, we use (5.6). Then

$$
\begin{gathered}
\left\{\Phi^{*} B_{i j}, \Phi^{*} a_{k}\right\}=-\sum_{m, n}\left\{u^{m j} \eta_{n m} u_{i n}, I_{k}\right\}=0, \\
\left\{\Phi^{*} B_{i j}, \Phi^{*} A_{k l}\right\}=-\sum_{m, n}\left\{u^{m j} \eta_{n m} u_{i n}, u_{k l}\right\}=-\sum_{m, n} u^{m j}\left\{\eta_{n m}, u_{k l}\right\} u_{i n} \\
=\sum_{m, n} u^{m j} \delta_{l n} u_{k m} u_{i n}=\delta_{j k} u_{i l}=\delta_{j k} \Phi^{*} A_{i l}, \\
\left\{\Phi^{*} B_{i j}, \Phi^{*} b_{k}\right\}=-\sum_{m, n, p}\left\{u^{m j} \eta_{n m} u_{i n}, J_{p} u_{k p}\right\}=-\sum_{m, n, p} u^{m j}\left\{\eta_{n m}, u_{k p}\right\} u_{i n} J_{p} \\
=\sum_{m, n, p} u^{m j} \delta_{p n} u_{k m} u_{i n} J_{p}=\delta_{j k} \sum_{p} u_{i p} J_{p}=\delta_{j k} \Phi^{*} b_{i}, \\
\left\{\Phi^{*} B_{i j}, \Phi^{*} B_{k l}^{\prime}\right\}=-\sum_{m, n}\left\{u^{m j} \eta_{n m} u_{i n}, \eta_{k l}-I_{l} J_{k}\right\} \\
=-\sum_{m, n}\left(\left\{u^{m j}, \eta_{k l}\right\} \eta_{n m} u_{i n}+u^{m j}\left\{\eta_{n m}, \eta_{k l}\right\} u_{i n}+u^{m j} \eta_{n m}\left\{u_{i n}, \eta_{k l}\right\}\right) \\
=\sum_{m, n}\left(\delta_{l m} u^{k j} \eta_{n m} u_{i n}-u^{m j}\left(\delta_{m k} \eta_{n l}-\delta_{l n} \eta_{k m}\right) u_{i n}-u^{m j} \eta_{n m} \delta_{k n} u_{i l}\right)=0 .
\end{gathered}
$$

For the remaining computation, let us note

$$
\left\{\Phi^{*} B_{i j}, \Phi^{*} B_{k l}\right\}=\sum_{m, n}\left\{\Phi^{*} B_{i j}, u^{m l} \eta_{n m} u_{k n}\right\}=\sum_{m, n}\left\{\Phi^{*} B_{i j}, \Phi^{*} A^{m l} \cdot \Phi^{*} B_{n m}^{\prime} \cdot \Phi^{*} A_{k n}\right\} .
$$

We substitute the above computation and

$$
\left\{\Phi^{*} B_{i j}, \Phi^{*} A^{m l}\right\}=-\delta_{i l} \Phi^{*} A^{m j} .
$$


We get

$$
\begin{aligned}
\left\{\Phi^{*} B_{i j}, \Phi^{*} B_{k l}\right\} & =\sum_{m, n}\left(-\delta_{i l} \Phi^{*} A^{m j} \cdot \Phi^{*} B_{n m}^{\prime} \cdot \Phi^{*} A_{k n}+\delta_{j k} \Phi^{*} A^{m l} \cdot \Phi^{*} B_{n m}^{\prime} \cdot \Phi^{*} A_{i n}\right) \\
& =\delta_{j k} \Phi^{*} B_{i l}-\delta_{i l} \Phi^{*} B_{k j} .
\end{aligned}
$$

Thus we have checked all relations in (5.2).

For the cases $\mathbf{v}_{1} \neq \mathbf{v}_{2}$, linear maps $A, B, B^{\prime}, a, b$ are blocks of $u, \eta$. Therefore required computation is already contained above. For example, when $\mathbf{v}_{1}>\mathbf{v}_{2}$, we have $a_{k}=\eta_{n k}$, where $n=\operatorname{dim} V$. Hence

$$
\left\{\Phi^{*} B_{i j}^{\prime}, \Phi^{*} a_{k}\right\}=\left\{\eta_{i j}, \eta_{n k}\right\}=-\delta_{i k} \eta_{n j}=-\delta_{i k} \Phi^{*} a_{j},
$$

as $1 \leq i, j \leq m$, hence $j \neq n$. We omit further detail.

\subsection{Poisson commutativity}

Thanks to Proposition 5.7, the Poisson structure on $\mathcal{M}$ is given by the reduction of the product of one in $\S 5.1$ (triangle part) and the standard one on the two way part. Therefore we can apply the computation in $[F R 14,(8)]$. Here is one example. More examples will be given in the proof of Proposition 6.20.

Proposition 5.8.

$$
\left\{\operatorname{tr}\left(B_{\zeta}^{k}\right), \operatorname{tr}\left(B_{\zeta^{\prime}}^{l}\right)\right\}=0 \quad \text { for segments } \zeta, \zeta^{\prime} .
$$

If $\zeta, \zeta^{\prime}$ are not equal or adjacent, the bracket is obviously 0 . If $\zeta, \zeta^{\prime}$ are adjacent, it is zero by (5.2). If $\zeta=\zeta^{\prime}$, it is zero by [FR14, Prop. 3.11].

\section{Bow varieties as Coulomb branches}

\subsection{Bow varieties associated with quiver gauge theories}

Let us consider a framed quiver gauge theory of an affine type $A_{n-1}$ with dimension vectors $\underline{\mathbf{v}}=\left(\mathbf{v}_{0}, \cdots, \mathbf{v}_{n-1}\right)$ and $\underline{\mathbf{w}}=\left(\mathbf{w}_{0}, \cdots, \mathbf{w}_{n-1}\right)$. The goal of this section is to show that the Coulomb branch $\mathcal{M}_{C}$ is the bow variety associated with

$$
\mathbf{v}_{n-2}^{\mathbf{v}_{n-1}} \times \underbrace{\mathbf{v}_{n-1} \bigcirc^{\mathbf{v}_{n-1}}}_{\mathbf{w}_{n-1}} \times \underbrace{\mathbf{v}_{0} \bigcirc^{\cdots} \mathrm{v}^{\mathbf{v}_{0}}}_{\mathbf{w}_{0}} \times{ }^{\mathbf{v}_{1}} .
$$

These bow varieties always have balanced dimension vectors. Conversely, balanced dimension vectors are determined by $\underline{\mathbf{v}}, \underline{\mathbf{w}}$. We denote the corresponding bow variety by $\mathcal{M}(\underline{\mathbf{v}}, \underline{\mathbf{w}})$. Unless explicitly written as $\mathcal{M}_{\nu}$, the parameter $\nu$ is set 0 . Let $\ell$ denote the level of $\underline{\mathbf{w}}$, i.e., $\ell=\sum_{i} \mathbf{w}_{i}$. We allow $\ell=0$, i.e., $\underline{\mathbf{w}}=0$ (see $\S 6.3$ ). But we do not consider the case $n=0($ no $\times$ ).

We put subscripts to endomorphisms in the quiver description of a bow variety as follows:
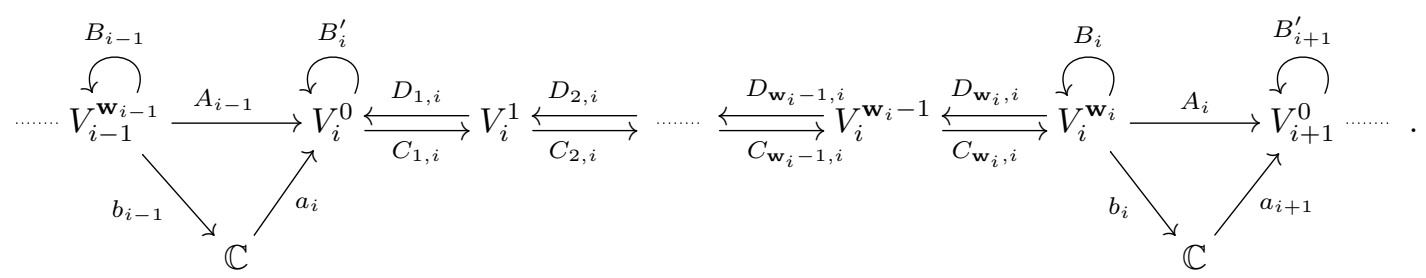


\subsection{Deformation and resolution}

\subsection{1 $\underline{\mathrm{w}}=0$ case}

When $\underline{\mathbf{w}}=0$, we do not have parameters $\nu$ as there is no two way part. But we can put parameters in a different way, following [FR14, §3.33].

Take $\nu^{\mathbb{C}}=\left(\nu_{0}^{\mathbb{C}}, \nu_{1}^{\mathbb{C}}, \ldots, \nu_{n-1}^{\mathbb{C}}\right) \in \mathbb{C}^{n}$.

$$
\widetilde{\mathcal{M}}_{\nu^{\mathbb{C}}}(\underline{\mathbf{v}}, 0)=\left\{\mu^{-1}\left(\nu^{\mathbb{C}}\right) \text { satisfying }(\mathrm{S} 1),(\mathrm{S} 2)\right\}, \quad \mathcal{M}_{\nu^{\mathbb{C}}}(\underline{\mathbf{v}}, 0)=\widetilde{\mathcal{M}}_{\nu^{\mathbb{C}}}(\underline{\mathbf{v}}, 0) / / \prod_{i=0}^{n-1} \mathrm{GL}\left(\mathbf{v}_{i}\right) .
$$

Here $\mu^{-1}\left(\nu^{\mathbb{C}}\right)$ means that $B_{i}^{\prime}$ is determined by $B_{i}-B_{i}^{\prime}=\nu_{i}^{\mathbb{C}} \mathrm{id}_{V_{i}}$.

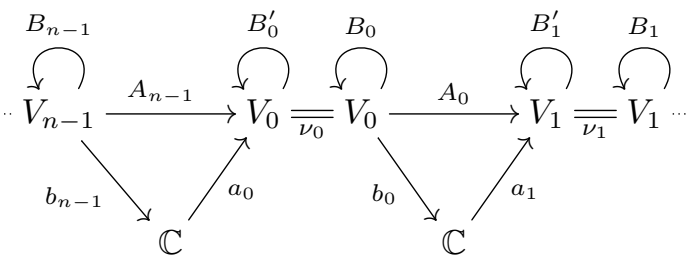

By the argument in $\S 3.1 .4$, we get

$$
\begin{aligned}
B_{1}^{\prime} A_{0}-A_{0} B_{0}+a_{1} b_{0}=0 & \Leftrightarrow\left(B_{1}-\nu_{1}^{\mathbb{C}} \mathrm{id}_{V_{1}}\right) A_{0}-A_{0} B_{0}+a_{1} b_{0}=0, \\
B_{2}^{\prime} A_{1}-A_{1} B_{1}+a_{2} b_{1}=0 \Leftrightarrow\left(B_{2}-\nu_{2}^{\mathbb{C}} \mathrm{id}_{V_{2}}\right) A_{1}-A_{1} B_{1}+a_{2} b_{1}=0 & \Leftrightarrow\left(B_{2}-\left(\nu_{1}^{\mathbb{C}}+\nu_{2}^{\mathbb{C}}\right) \mathrm{id}_{V_{2}}\right) A_{1}-A_{1}\left(B_{1}-\nu_{1}^{\mathbb{C}} \operatorname{id}_{V_{1}}\right)+a_{2} b_{1}=0,
\end{aligned}
$$

and so on. Thus only the sum $\sum_{k} \nu_{k}^{\mathbb{C}}$ matters. It means that this deformation forms at most a 1-dimensional family. Therefore we normalize as $\nu_{0}^{\mathbb{C}}=\nu_{1}^{\mathbb{C}}=\cdots=\nu_{n-1}^{\mathbb{C}}=-\nu_{*}^{\mathbb{C}}$ for a $\nu_{*}^{\mathbb{C}} \in \mathbb{C}$ hereafter.

When $n=1$, the defining equation is

$$
[B, A]+\nu_{*}^{\mathbb{C}} A+a b=0 .
$$

This is the trigonometric Calogero-Moser space [Obl04, §7.3].

When some $\mathbf{v}$ is 0 , this deformation becomes trivial because of $\mathrm{id}_{0}=0$. We expect that it is nontrivial otherwise.

Remark 6.2. This deformation is expected to be the 'dual' to the $\mathbb{C}^{\times}$-action for $\mathfrak{M}(\underline{\mathbf{v}}, 0), B \mapsto t B$ for $B \in \mathbf{N}, B \mapsto t^{-1} B$ for $B \in \mathbf{N}^{*}$ and $t \in \mathbb{C}^{*}$. See the construction in $\S 6.8 .2$. When some $\mathbf{v}$ is 0 , this action also becomes trivial.

Like this, $\mathcal{M}(\underline{\mathbf{v}}, 0)$ has a 1-parameter family given by the real parameter of the hyper-Kähler quotient in the original definition of a bow variety $\$ 2.1$. This family corresponds to a resolution in the quiver description of a bow variety. Concretely depending on the sign of $\sum \nu_{k}$, we impose either of (C-S1) or (C-S2) below. This can be checked as in $\S 2.4 .2$. We leave the details to the reader as an exercise.

\subsection{2 $\underline{\mathrm{w}} \neq 0$ case}

In this case, $\mathcal{M}(\underline{\mathbf{v}}, \underline{\mathbf{w}})$ has a $|\underline{\mathbf{w}}|$-dimensional family of the deformation, which is defined in $\S 2.2$.

For a later purpose, we shift parameters slightly. Let us introduce complex parameters as $\nu_{1, i}^{\mathbb{C}}$, $\ldots, \nu_{\mathbf{w}_{i}, i}^{\mathbb{C}}(i=0,1, \ldots, n-1), \nu_{*}^{\mathbb{C}}$ and consider deformation of the defining equations given by

$$
\begin{gathered}
B_{i}^{\prime} A_{i-1}-A_{i-1} B_{i-1}+a_{i} b_{i-1}=0, \quad-D_{1, i} C_{1, i}-B_{i}^{\prime}=-\nu_{*}^{\mathbb{C}}-\nu_{1, i}^{\mathbb{C}}, \\
-D_{k+1, i} C_{k+1, i}+C_{k, i} D_{k, i}=\nu_{k, i}^{\mathbb{C}}-\nu_{k+1, i}^{\mathbb{C}} \quad\left(k=1, \ldots, \mathbf{w}_{i}-1\right), \quad C_{\mathbf{w}_{i}, i} D_{\mathbf{w}_{i}, i}+B_{i}=\nu_{\mathbf{w}_{i}, i}^{\mathbb{C}} .
\end{gathered}
$$

If we make shift $\nu_{k, i}^{\mathbb{C}} \mapsto \nu_{k, i}^{\mathbb{C}}+s$ for a fixed $s$, keeping $\nu_{*}^{\mathbb{C}}$, it yields a trivial deformation as it is same as shifts of $B_{i}$. Therefore we have $|\underline{\mathbf{w}}|$-dimensional family. 


\subsection{Chainsaw quiver variety}

We consider the $\underline{\mathbf{w}}=0$ case. The corresponding quiver is

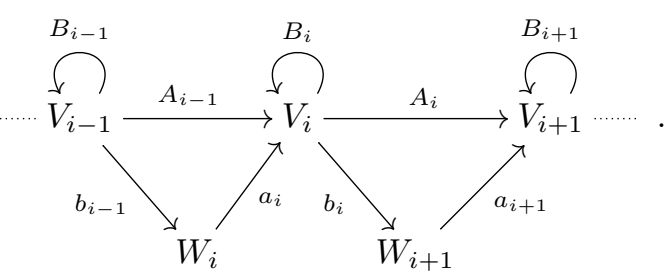

This quiver is called a chainsaw quiver, and studied in [FR14]. $\mathcal{M}(\underline{\mathbf{v}}, 0)$ does not coincide with the variety $\mathfrak{Z}_{d}$ in [FR14] in general, as we impose the conditions (S1), (S2). Here the variety $\mathfrak{Z}_{d}$ is the categorical quotient of $\mu^{-1}(0)$ by $\mathcal{G}=\prod \mathrm{GL}\left(\mathbf{v}_{i}\right)$. Let us denote it by $\overline{\mathcal{M}}(\underline{\mathbf{v}}, 0)$. Our $\mathcal{M}(\underline{\mathbf{v}}, 0)$ is an open subvariety of $\overline{\mathcal{M}}(\underline{\mathbf{v}}, 0)$, denoted by $\check{Z}^{\alpha}$ in [BFN16b, $\S 3$ (viii)].

Let us assume $\nu_{*}^{\mathbb{C}}=0$ during the rest of this subsection.

Proposition 6.4 ([Tak16, Cor. 3.9]). $\mathcal{M}^{\mathrm{reg}}(\underline{\mathbf{v}}, 0)$ is the quotient of the open subvariety of $\mu^{-1}(0)$ consisting of points satisfying

$$
\begin{aligned}
& (\mathrm{C}-\mathrm{S} 1): B_{i}\left(S_{i}\right) \subset S_{i}, S_{i} \subset \operatorname{Ker} b_{i}, A_{i}\left(S_{i}\right) \subset S_{i+1} \Rightarrow S_{i}=0, \\
& (\mathrm{C}-\mathrm{S} 2): B_{i}\left(T_{i}\right) \subset T_{i}, T_{i} \supset \operatorname{Im} a_{i}, A_{i}\left(T_{i}\right) \subset T_{i+1} \Rightarrow T_{i}=V_{i},
\end{aligned}
$$

by the action of $\mathcal{G}$.

The conditions (C-S1) and (C-S2) coincides with the 0-stability condition, in the meaning of King's stability [Kin94].

A chainsaw quiver variety is related to other important objects:

Proposition 6.5. (1) (c.f. [Tak16, Th. 3.5]) $\mathcal{M}(\underline{\mathbf{v}}, 0)$ is isomorphic to a moduli spaces of solutions of Nahm's equations over $S^{1}$.

(2) [Tak16, Th. 4.2] $\mathcal{M}^{\mathrm{reg}}(\underline{\mathbf{v}}, 0)$ is isomorphic to a framed moduli space of locally free parabolic sheaves over $\mathbb{P}^{1} \times \mathbb{P}^{1}$.

(3) $\mathcal{M}(\underline{\mathbf{v}}, 0) \cong \bigsqcup_{k} \mathcal{M}^{\mathrm{reg}}(\underline{\mathbf{v}}-k \cdot \underline{1}, 0) \times S^{k}\left(\mathbb{C} \times \mathbb{C}^{\times}\right)$.

Proof. We prove the third assertion. Notice that each triangle of $\mathcal{M}(\underline{\mathbf{v}}, 0)$ satisfies the conditions (S1), (S2). Suppose that there exist subspaces $\left\{S_{i}\right\}$ and $\left\{T_{i}\right\}$ as in (C-S1) and (C-S2) respectively. Proposition 2.2 means that $\operatorname{dim} S_{i} \leq \operatorname{dim} S_{i+1}$ and $\operatorname{codim} T_{i} \geq \operatorname{codim} T_{i+1}$. Thus we have $\operatorname{dim} S_{i}=$ $\operatorname{dim} S_{i+1}$ and $\operatorname{codim} T_{i}=\operatorname{codim} T_{i+1}$ for any $i$.

As in the proof of Lemma 4.1, a point in $\mathcal{M}(\underline{\mathbf{v}}, 0)$ decomposes into a direct sum of an object satisfying (C-S1), (C-S2) and those with $a=b=0$ and $A$ is an isomorphism. A summand of the second type must have $\operatorname{dim} S_{i}=1$ for any $i$ if it is not further decomposable. We can normalize $A$ 's to id except for one. Then all $B$ are equal. Therefore the data corresponds to a point in $\mathbb{C} \times \mathbb{C}^{\times}$.

\subsection{Collapsing morphism to a chainsaw quiver variety}

We construct a collapsing morphism $s: \mathbb{M}(\underline{\mathbf{v}}, \underline{\mathbf{w}}) \rightarrow \mathbb{M}(\underline{\mathbf{v}}, 0)$ as follows:

(6.6) $\quad A_{i}^{\text {new }}:=A_{i} C_{\mathbf{w}_{i} \cdots 1, i}, \quad B_{i}^{\text {new }}:=B_{i}^{\prime}=\nu_{1, i}^{\mathbb{C}}+\nu_{*}^{\mathbb{C}}-D_{1, i} C_{1, i}, \quad a_{i}^{\text {new }}:=a_{i}, \quad b_{i}^{\text {new }}:=b_{i} C_{\mathbf{w}_{i} \cdots 1, i}$,

where $C_{\mathbf{w}_{i} \cdots 1, i}$ means the composite $C_{\mathbf{w}_{i}, i} \cdots C_{1, i}$ as before. The new data $\left(A_{i}^{\text {new }}, B_{i}^{\text {new }}, a_{i}^{\text {new }}, b_{i}^{\text {new }}\right)$ satisfy the equation for $\operatorname{Hom}\left(V_{i}, V_{i+1}\right)$ as

$$
\begin{aligned}
& B_{i+1}^{\text {new }} A_{i}^{\text {new }}-A_{i}^{\text {new }} B_{i}^{\text {new }}+\nu_{*}^{\mathbb{C}} A_{i}^{\text {new }}+a_{i+1}^{\text {new }} b_{i}^{\text {new }} \\
& =B_{i+1}^{\prime} A_{i} C_{\mathbf{w}_{i} \cdots 1, i}+A_{i} C_{\mathbf{w}_{i} \cdots 1, i}\left(D_{1, i} C_{1, i}-\nu_{1, i}^{\mathbb{C}}\right)+a_{i+1} b_{i} C_{\mathbf{w}_{i} \cdots 1, i} \\
& =\left(B_{i+1}^{\prime} A_{i}-A_{i} B_{i}+a_{i+1} b_{i}\right) C_{\mathbf{w}_{i} \cdots 1, i}=0 .
\end{aligned}
$$


Obviously, this morphism descends to $s: \mathcal{M}(\underline{\mathbf{v}}, \underline{\mathbf{w}}) \rightarrow \overline{\mathcal{M}}(\underline{\mathbf{v}}, 0)$, but $s(\mathcal{M}(\underline{\mathbf{v}}, \underline{\mathbf{w}}))$ is not contained in $\mathcal{M}(\underline{\mathbf{v}}, 0)$. This is because (S1) for $\left(A_{i}^{\text {new }}, B_{i}^{\text {new }}, a_{i}^{\text {new }}, b_{i}^{\text {new }}\right)$ leads to $\operatorname{Ker} C_{1, i}=0$, which is not true in general.

Proposition 6.7. The collapsing morphism s induces an isomorphism $s^{-1}(\mathcal{M}(\underline{\mathbf{v}}, 0)) \stackrel{\cong}{\longrightarrow} \mathcal{M}(\underline{\mathbf{v}}, 0)$. Hence we have an open embedding $\mathcal{M}(\underline{\mathbf{v}}, 0) \hookrightarrow \mathcal{M}(\underline{\mathbf{v}}, \underline{\mathbf{w}})$.

Proof. Since $B_{i}^{\prime}=\nu_{1, i}^{\mathbb{C}}+\nu_{*}^{\mathbb{C}}-D_{1, i} C_{1, i}$, we have $C_{1, i}\left(B_{i}^{\prime}-\nu_{*}^{\mathbb{C}}\right)=\left(\nu_{2, i}^{\mathbb{C}}-D_{2, i} C_{2, i}\right) C_{1, i}$, and $C_{2, i} C_{1, i}\left(B_{i}^{\prime}-\nu_{*}^{\mathbb{C}}\right)=\left(\nu_{3, i}^{\mathbb{C}}-D_{3, i} C_{3, i}\right) C_{2, i} C_{1, i}$, and so on. Finally we get $C_{\mathbf{w}_{i} \cdots 1, i}\left(B_{i}^{\prime}-\nu_{*}^{\mathbb{C}}\right)=$ $B_{i} C_{\mathbf{w}_{i} \cdot 1, i}$. Therefore $\operatorname{Ker} C_{\mathbf{w}_{i} \cdot 1, i}$ is contained in $\operatorname{Ker} b_{i}^{\text {new }}$ and invariant under $B_{i}^{\text {new }}$. Therefore (S1) for $\left(A^{\text {new }}, B^{\text {new }}, a^{\text {new }}, b^{\text {new }}\right)$ implies the injectivity of $C_{\mathbf{w}_{i} \cdot 1, i}$. Since the balanced condition is satisfied, all $C_{1, i}, C_{2, i}, \ldots$ are isomorphisms. By the action of $\operatorname{GL}\left(V_{i}^{1}\right) \times \cdots \times \operatorname{GL}\left(V_{i}^{\mathbf{w}_{i}}\right)$, we normalize $C_{1, i}, C_{2, i}, \ldots$ as the identity. Then $D_{1, i}, D_{2, i}, \ldots$ are determined by the defining equations $B_{i}=\nu_{\mathbf{w}_{i}, i}^{\mathbb{C}}-D_{1, i} C_{1, i}, D_{2, i} C_{2, i}=C_{1, i} D_{1, i}-\nu_{1, i}^{\mathbb{C}}+\nu_{2, i}^{\mathbb{C}}$, and so on. Hence the original data is recovered from the new one.

Proposition 6.8. The collapsing morphism $s$ is Poisson.

Proof. Recall $\mathcal{M}(\underline{\mathbf{v}}, \underline{\mathbf{w}})$ is a reduction of $\widetilde{\mathcal{M}}_{\text {sym }}$ by $\mathcal{G}=\prod \operatorname{GL}\left(V_{\zeta}\right)$. On the other hand $\overline{\mathcal{M}}(\underline{\mathbf{v}}, 0)$ is also a reduction of $\widetilde{\mathcal{M}^{\prime}}$, the product of $\mu^{-1}(0)$ in $\S 5.1$ for each triangle. (It is the reduction as a Poisson variety.)

The morphism $s$ is induced from the corresponding morphism $\widetilde{s}: \widetilde{\mathcal{M}}_{\text {sym }} \rightarrow \widetilde{\mathcal{M}}^{\prime}$. Therefore it is enough to check that $\widetilde{s}$ is Poisson. But this is clear from the definition of $\widetilde{s}$ given by the formula (6.6).

\subsection{Coordinate and Local models}

We order eigenvalues of $B_{i}$, and denote them by $\left(w_{i, 1}, \cdots, w_{i, \mathbf{v}_{i}}\right)$. Define

$$
\begin{aligned}
& y_{i, k}:=b_{i} \prod_{\substack{1 \leq l \leq \mathbf{v}_{i} \\
l \neq k}}\left(B_{i}-w_{i, l} \mathrm{id}\right) C_{\mathbf{w}_{i} \cdots 1, i} a_{i} \quad(n>1), \\
& y_{k}:=\operatorname{tr}\left[A_{0} \prod_{\substack{1 \leq l \leq \mathbf{v}_{0} \\
l \neq k}}\left(B_{0}-w_{l} \mathrm{id}\right) C_{\mathbf{w}_{0} \cdots 1,0}\right] \quad(n=1) .
\end{aligned}
$$

This definition coincides with that of $[\mathrm{FR} 14, \S 3.4]$ when $\mathbf{w}_{i}=0$. These are considered as coordinates of $\mathcal{M}(\underline{\mathbf{v}}, \underline{\mathbf{w}}) \times_{\mathbb{A}} \underline{\mathbb{A}} \mathbb{A}^{|\mathbf{v}|}$.

In the remainder of this subsection, we study some examples of $\mathcal{M}(\underline{\mathbf{v}}, \underline{\mathbf{w}})$ and $\overline{\mathcal{M}}(\underline{\mathbf{v}}, 0)$. We will be interested in affine algebraic varieties, hence set $\nu^{\mathbb{R}}=0$.

6.5.1 $n=1, \mathbf{v}=1$

Let us first consider the case $\nu^{\mathbb{C}}=0$.
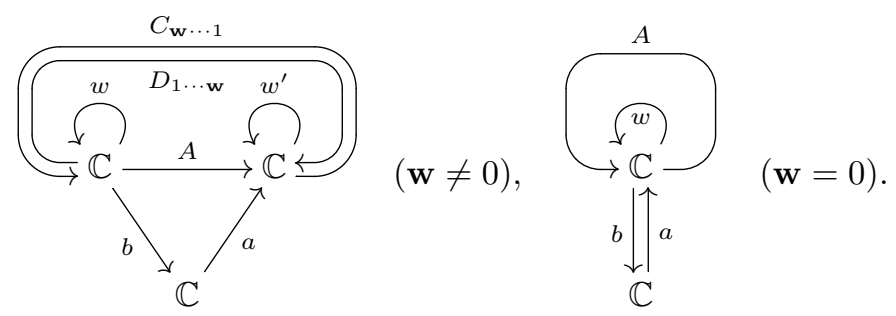

We have $w^{\prime}=-D_{1} C_{1}=\cdots=-C_{\mathbf{w}} D_{\mathbf{w}}=w$. Then $B_{2} A-A B_{1}+a b=0$ means $a b=0$, and in closed orbits we can take a representative $a=b=0, A=1$ when $\mathbf{w} \neq 0$. We have $y=C_{\mathbf{w} \cdots 1}$. Let us set $x=(-1)^{\mathbf{w}} D_{1 \cdots \mathbf{w}}$. Then we get $x y=w^{\mathbf{w}}$ because $w=-D_{1} C_{1}$, so

$$
\mathbb{C}[\mathcal{M}(1, \mathbf{w})]=\mathbb{C}[w, y, x] /\left(x y-w^{\mathbf{w}}\right) .
$$


When $\mathbf{w}=0, y:=A$ becomes invertible if and only if (S1) and (S2) are imposed, thus we get

$$
\mathbb{C}[\mathcal{M}(1,0)]=\mathbb{C}\left[w, y^{ \pm 1}\right], \quad \mathbb{C}[\overline{\mathcal{M}}(1,0)]=\mathbb{C}[w, y] .
$$

We consider complex parameter $\left(\nu_{1}^{\mathbb{C}}, \ldots, \nu_{\mathbf{w}}^{\mathbb{C}}, \nu_{*}^{\mathbb{C}}\right)$ as above. Then $w=\nu_{\mathbf{w}}^{\mathbb{C}}-C_{\mathbf{w}} D_{\mathbf{w}}=\nu_{\mathbf{w}-1}^{\mathbb{C}}-$ $C_{\mathbf{w}-1} D_{\mathbf{w}-1}=\cdots=\nu_{1}^{\mathbb{C}}-C_{1} D_{1}=w^{\prime}-\nu_{*}^{\mathbb{C}}$. Thus we change $a b=0$ to $\nu_{*}^{\mathbb{C}} A+a b=0$.

Suppose $\mathbf{w} \neq 0$. Since $A=0$ contradicts with (S1),(S2), we can normalize $A=1$. We can furthre normalize $a, b$ by $\mathbb{C}^{\times}$-action and the equation $a b=-\nu_{*}^{\mathbb{C}}$. The defining relation is perturbed as $x y=\left(w-\nu_{1}^{\mathbb{C}}\right) \ldots\left(w-\nu_{\mathbf{w}}^{\mathbb{C}}\right)$. The overall shift $\left(\nu_{1}^{\mathbb{C}}, \ldots, \nu_{\mathbf{w}}^{\mathbb{C}}\right) \mapsto\left(\nu_{1}^{\mathbb{C}}+s, \ldots, \nu_{\mathbf{w}}^{\mathbb{C}}+s\right)(s \in \mathbb{C})$ and $\nu_{*}^{\mathbb{C}}$ are trivial deformation, and other directions are nontrivial.

When $\mathbf{w}=0$, we set $y:=A$ as above. It is invertible thanks to (S1),(S2). Once $y$ is fixed, $a, b$ are also fixed by the equation and the $\mathbb{C}^{\times}$-action. Hence $\mathbb{C}\left[\mathcal{M}_{\nu}(1,0)\right]=\mathbb{C}\left[w, y^{ \pm}\right]$.

6.5.2 $n=1, \mathbf{v}=2$
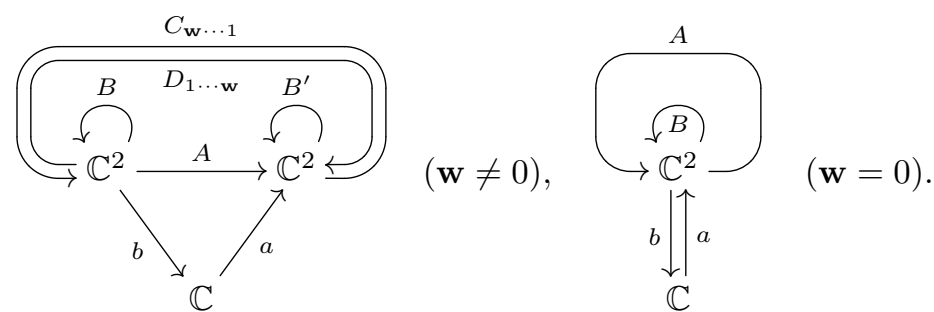

If $\mathbf{w} \neq 0$, the bow variety is isomorphic to the affine type $A_{\mathbf{w}-1}$ quiver variety $\mathfrak{M}_{\nu \mathbb{c}}\left(\underline{\mathbf{v}}^{\prime}, \underline{\mathbf{w}}^{\prime}\right)$ with dimension vectors $\underline{\mathbf{v}}^{\prime}=(2, \cdots, 2), \underline{\mathbf{w}}^{\prime}=(1,0, \cdots, 0)$ by Theorem 2.15. (The parameter is shifted as in (6.3).) Further more, it is well-known

$$
\mathcal{M}(2, \mathbf{w}) \cong \mathfrak{M}_{0}\left(\underline{\mathbf{v}}^{\prime}, \underline{\mathbf{w}}^{\prime}\right) \cong S^{2}\left(\mathbb{C}^{2} / \mathbb{Z}_{\mathbf{w}}\right)
$$

for $\nu^{\mathbb{C}}=0$. (See e.g., [Nak99, 2.10] for $\mathbf{w}=1$ : We can set $A=\mathrm{id}, a=b=0$, and $C=\operatorname{diag}\left(y_{1}, y_{2}\right)$, $\left.D=\operatorname{diag}\left(x_{1}, x_{2}\right), B=D C=\operatorname{diag}\left(w_{1}, w_{2}\right).\right)$ If $\nu_{*}^{\mathbb{C}}=0$, it is the second symmetric product of $x y=\left(w-\nu_{1}^{\mathbb{C}}\right)\left(w-\nu_{2}^{\mathbb{C}}\right) \cdots\left(w-\nu_{\mathbf{w}_{1}}^{\mathbb{C}}\right)$. If $\nu_{*}^{\mathbb{C}} \neq 0$, it is a deformation of the symmetric product.

If $\mathbf{w}=0$, we again use $[\mathrm{Nak} 99,2.10]$, and impose that $A$ is invertible. Hence

$\mathbb{C}[\mathcal{M}(2,0)] \cong \mathbb{C}\left[w_{1}, w_{2}, y_{1}^{ \pm}, y_{2}^{ \pm}\right]^{\mathfrak{S}_{2}}=\mathbb{C}\left[S^{2}\left(\mathbb{C} \times \mathbb{C}^{\times}\right)\right], \mathbb{C}[\overline{\mathcal{M}}(2,0)] \cong \mathbb{C}\left[w_{1}, w_{2}, y_{1}, y_{2}\right]^{\mathfrak{S}_{2}}=\mathbb{C}\left[S^{2}\left(\mathbb{C}^{2}\right)\right]$ for $\nu_{*}^{\mathbb{C}}=0$. For general $\nu_{*}^{\mathbb{C}}$, the defining equation is

$$
[B, A]+\nu_{*}^{\mathbb{C}} A+a b=0 .
$$

This is the trigonometric Calogero-Moser space [Ob104, §7.3].

6.5.3 $n=2, \underline{\mathbf{v}}=(1,0)$

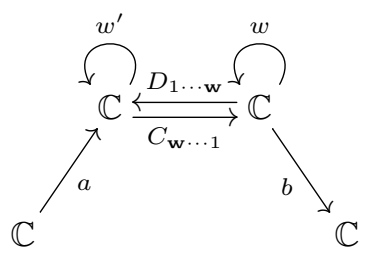

The conditions (S1) and (S2) mean $a, b \in \mathbb{C}^{\times}$, so we can take a representation $a=1=b$ when $\mathbf{w} \neq 0$. Then we have $y=C_{\mathbf{w} \cdots 1}$ by definition and set $x=(-1)^{w} D_{1 \cdots \mathbf{w}}$. Since we again get $x y=\left(w-\nu_{1}^{\mathbb{C}}\right) \ldots\left(w-\nu_{\mathbf{w}}^{\mathbb{C}}\right)$, so

$$
\mathbb{C}[\mathcal{M}((1,0),(\mathbf{w}, 0))]=\mathbb{C}[w, y, x] /\left(x y-\left(w-\nu_{1}^{\mathbb{C}}\right) \ldots\left(w-\nu_{\mathbf{w}}^{\mathbb{C}}\right)\right) .
$$


When $\mathbf{w}=0$, we have $y=b a$ by definition and $y \in \mathbb{C}^{\times}$if and only if (S1) and (S2) are imposed:

$$
\mathbb{C}[\mathcal{M}((1,0), 0)]=\mathbb{C}\left[w, y^{ \pm 1}\right], \quad \mathbb{C}[\overline{\mathcal{M}}((1,0), 0)]=\mathbb{C}[w, y]
$$

Furthermore, the collapsing morphism is described as

$$
s: \mathcal{M}((1,0),(\mathbf{w}, 0)) \rightarrow \overline{\mathcal{M}}((1,0), 0),(w, y, x) \mapsto(w, y)
$$

6.5.4 $n=2, \underline{\mathbf{v}}=(1,1)$

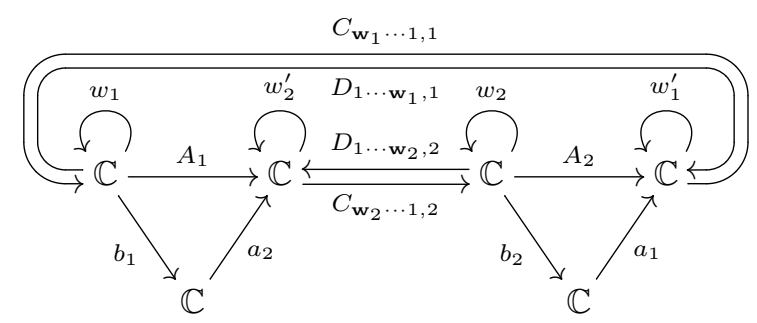

We have $y_{1}=b_{1} C_{\mathbf{w}_{1} \cdots 1,1} a_{1}$ and $y_{2}=b_{2} C_{\mathbf{w}_{2} \cdots 1,2} a_{2}$ by definition. By Lemma 3.1, $A_{1}$ and $A_{2}$ are invertible, so we set $y_{12}=-A_{1}^{-1} D_{1 \cdots \mathbf{w}_{2}, 2} A_{2}^{-1} D_{1 \cdots \mathbf{w}_{1}, 1}$. Let $f_{1}\left(w_{1}\right):=\left(w_{1}-\nu_{1,1}^{\mathbb{C}}\right) \ldots\left(w_{1}-\nu_{\mathbf{w}_{1}, 1}^{\mathbb{C}}\right)$, $f_{2}\left(w_{2}\right):=\left(w_{2}-\nu_{1,2}^{\mathbb{C}}\right) \ldots\left(w_{2}-\nu_{\mathbf{w}_{2}, 2}^{\mathbb{C}}\right)$. Note also $w_{1}^{\prime}=w_{1}+\nu_{*}^{\mathbb{C}}, w_{2}^{\prime}=w_{2}+\nu_{*}^{\mathbb{C}}$. Since

$$
\begin{aligned}
A_{1} A_{2} & \left(y_{1} y_{2} y_{12}-f_{1}\left(w_{1}\right) f_{2}\left(w_{2}\right)\left(w_{1}-w_{2}+\nu_{*}^{\mathbb{C}}\right)\left(w_{1}-w_{2}-\nu_{*}^{\mathbb{C}}\right)\right) \\
& =-b_{1} a_{1} C_{\mathbf{w}_{1} \cdots 1,1} D_{1 \cdots \mathbf{w}_{1}, 1} b_{2} a_{2} C_{\mathbf{w}_{2} \cdots 1,2} D_{1 \cdots \mathbf{w}_{2}, 2}+f_{1}\left(w_{1}\right) f_{2}\left(w_{2}\right) A_{1}\left(w_{2}^{\prime}-w_{1}\right) A_{2}\left(w_{1}^{\prime}-w_{2}\right) \\
& =-b_{1} a_{1} b_{2} a_{2} f_{1}\left(w_{1}\right) f_{2}\left(w_{2}\right)+f_{1}\left(w_{1}\right) f_{2}\left(w_{2}\right)\left(-a_{1} b_{2}\right)\left(-a_{2} b_{1}\right)=0
\end{aligned}
$$

we have

$$
\begin{aligned}
\mathbb{C}[\mathcal{M}((1,1), & \left.\left.\left(\mathbf{w}_{1}, \mathbf{w}_{2}\right)\right)\right] \\
& =\mathbb{C}\left[w_{1}, w_{2}, y_{1}, y_{2}, y_{12}\right] /\left(y_{1} y_{2} y_{12}-f_{1}\left(w_{1}\right) f_{2}\left(w_{2}\right)\left(w_{1}-w_{2}+\nu_{*}^{\mathbb{C}}\right)\left(w_{1}-w_{2}-\nu_{*}^{\mathbb{C}}\right)\right) .
\end{aligned}
$$

When $\mathbf{w}_{1}=\mathbf{w}_{2}=0, y_{12}^{-1}$ becomes $-A_{1} A_{2}$, so we get

$$
\begin{aligned}
& \mathbb{C}[\mathcal{M}((1,1), 0)]=\mathbb{C}\left[w_{1}, w_{2}, y_{1}, y_{2}, y_{12}^{ \pm 1}\right] /\left(y_{1} y_{2}-y_{12}^{-1}\left(w_{1}-w_{2}\right)^{2}\right), \\
& \mathbb{C}[\overline{\mathcal{M}}((1,1), 0)]=\mathbb{C}\left[w_{1}, w_{2}, y_{1}, y_{2}, y_{12}^{-1}\right] /\left(y_{1} y_{2}-y_{12}^{-1}\left(w_{1}-w_{2}\right)^{2}\right)
\end{aligned}
$$

if $\nu_{*}^{\mathbb{C}}=0$. For general $\nu_{*}^{\mathbb{C}}$, we impose $w_{1}-w_{1}^{\prime}=-\nu_{*}^{\mathbb{C}}, w_{2}-w_{2}^{\prime}=-\nu_{*}^{\mathbb{C}}$. Hence the defining equation is

$$
y_{1} y_{2}-y_{12}^{-1}\left(w_{1}-w_{2}+\nu_{*}^{\mathbb{C}}\right)\left(w_{1}-w_{2}-\nu_{*}^{\mathbb{C}}\right)=0 .
$$

6.5.5 $n=2, \underline{\mathbf{v}}=(2,0)$

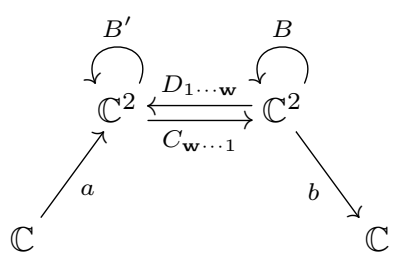

First we consider the $\mathbf{w} \neq 0$ case. Note $\operatorname{tr}\left(B^{\prime k}\right)=\operatorname{tr}\left(\left(\nu_{*}^{\mathbb{C}}+B\right)^{k}\right)$ for $k=1,2$ by defining equations. When $B$ has only one eigenvalue, $B$ (resp. $B^{\prime}$ ) must be equivalent to the Jordan form with size 2 because of the condition (S2) (resp. (S1)). So $B+\nu_{k}^{\mathbb{C}}$ and $B^{\prime}$ are always equivalent. Using 
Corollary 3.5 and Proposition 3.2(1), we can identify two triangle parts. Thus the bow variety is isomorphic to affine type $A_{\mathbf{w}-1}$ quiver variety $\mathfrak{M}_{\nu}\left(\underline{\mathbf{v}}^{\prime}, \underline{\mathbf{w}}^{\prime}\right)$ (see also 6.5.2, [Nak99, Lem. 2.9, Th. 3.24]):
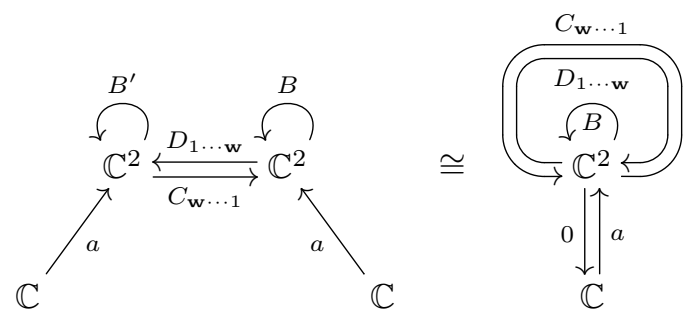

And it is a deformation of $S^{2}\left(\mathbb{C}^{2} / \mathbb{Z}_{\mathbf{w}}\right)$

When $\mathbf{w}=0$, we have $y_{1}=b\left(B-w_{2}\right) a$ and $y_{2}=b\left(B-w_{1}\right) a$ by definition. Here we can see $y_{i}$ is invertible if and only if (S1) and (S2) are imposed. Set $\xi=b a$. Since

$$
y_{1}-y_{2}-\xi\left(w_{1}-w_{2}\right)=b\left(B-w_{2}\right) a-b\left(B-w_{1}\right) a-b a\left(w_{1}-w_{2}\right)=0,
$$

we have

$$
\begin{aligned}
& \mathbb{C}\left[\mathcal{M}((2,0), 0) \times_{\mathbb{A}^{|2|}} \mathbb{A}^{2}\right]=\mathbb{C}\left[w_{1}, w_{2}, y_{1}^{ \pm 1}, y_{2}^{ \pm 1}, \xi\right] /\left(y_{1}-y_{2}-\xi\left(w_{1}-w_{2}\right)\right), \\
& \mathbb{C}\left[\overline{\mathcal{M}}((2,0), 0) \times_{\mathbb{A}^{|2|}} \mathbb{A}^{2}\right]=\mathbb{C}\left[w_{1}, w_{2}, y_{1}, y_{2}, \xi\right] /\left(y_{1}-y_{2}-\xi\left(w_{1}-w_{2}\right)\right) .
\end{aligned}
$$

See also [BFN16a, Ex. 3.20].

6.5.6 $n=3, \underline{\mathbf{v}}=(1,1,0)$

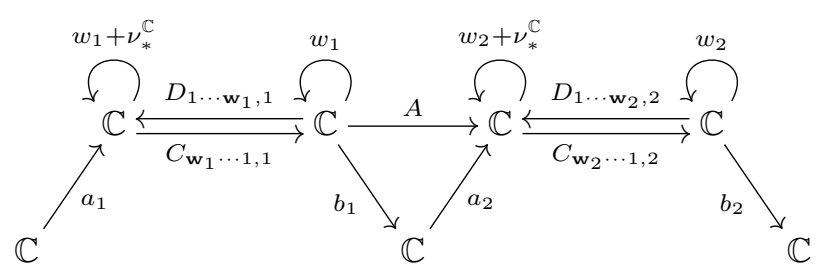

We have $y_{1}=b_{1} C_{\mathbf{w}_{1} \cdots 1,1} a_{1}$ and $y_{2}=b_{2} C_{\mathbf{w}_{2} \cdots 1,2} a_{2}$ by definition. Here, (S1) and (S2) mean $a_{1}, b_{2}, A \in \mathbb{C}^{\times}$and we normalize $a_{1}=1, b_{2}=1$ by the action of the leftmost and rightmost $\mathbb{C}^{\times}$. Set $y_{12}=D_{1 \cdots \mathbf{w}_{1}, 1} A^{-1} D_{1 \cdots \mathbf{w}_{2}, 2}$. Let $f_{1}\left(w_{1}\right):=\left(w_{1}-\nu_{1,1}^{\mathbb{C}}\right) \ldots\left(w_{1}-\nu_{\mathbf{w}_{1}, 1}^{\mathbb{C}}\right), f_{2}\left(w_{2}\right):=$ $\left(w_{2}-\nu_{1,2}^{\mathbb{C}}\right) \ldots\left(w_{2}-\nu_{\mathbf{w}_{2}, 2}^{\mathbb{C}}\right)$ as before. Since

$$
\begin{aligned}
& A\left\{y_{1} y_{2} y_{12}-f_{1}\left(w_{1}\right) f_{2}\left(w_{2}\right)\left(w_{1}-w_{2}-\nu_{*}^{\mathbb{C}}\right)\right\} \\
= & b_{1} C_{\mathbf{w}_{1} \cdots 1,1} C_{\mathbf{w}_{2} \cdots 1,2} a_{2} D_{1 \cdots \mathbf{w}_{1}, 1} D_{1 \cdots \mathbf{w}_{2}, 2}+f_{1}\left(w_{1}\right) f_{2}\left(w_{2}\right) A\left(w_{2}+\nu_{*}^{\mathbb{C}}-w_{1}\right) \\
= & b_{1} a_{2} f_{1}\left(w_{1}\right) f_{2}\left(w_{2}\right)+f_{1}\left(w_{1}\right) f_{2}\left(w_{2}\right)\left(-a_{2} b_{1}\right)=0,
\end{aligned}
$$

we have

$$
\mathbb{C}[\mathcal{M}((1,1,0), \underline{\mathbf{w}})]=\mathbb{C}\left[w_{1}, w_{2}, y_{1}, y_{2}, y_{12}\right] /\left(y_{1} y_{2} y_{12}-f_{1}\left(w_{1}\right) f_{2}\left(w_{2}\right)\left(w_{1}-w_{2}-\nu_{*}^{\mathbb{C}}\right)\right) .
$$

When $\mathbf{w}_{1}=\mathbf{w}_{2}=0, y_{12}^{-1}=A$, so we get

$$
\begin{aligned}
& \mathbb{C}[\mathcal{M}((1,1,0), 0)]=\mathbb{C}\left[w_{1}, w_{2}, y_{1}, y_{2}, y_{12}^{ \pm 1}\right] /\left(y_{1} y_{2}-y_{12}^{-1}\left(w_{1}-w_{2}-\nu_{*}^{\mathbb{C}}\right)\right), \\
& \mathbb{C}[\overline{\mathcal{M}}((1,1,0), 0)]=\mathbb{C}\left[w_{1}, w_{2}, y_{1}, y_{2}, y_{12}^{-1}\right] /\left(y_{1} y_{2}-y_{12}^{-1}\left(w_{1}-w_{2}-\nu_{*}^{\mathbb{C}}\right)\right) .
\end{aligned}
$$

The direction of $\nu_{*}^{\mathbb{C}}$ is a trivial deformation as expected. 


\subsection{Factorization property}

We can define $\Psi: \mathbb{M}(\underline{\mathbf{v}}, \underline{\mathbf{w}}) \rightarrow \mathbb{A} \underline{\mathbf{v}}$. And for $\underline{\mathbf{v}}=\underline{\mathbf{v}}^{\prime}+\underline{\mathbf{v}}^{\prime \prime}$, take $\left(\mathbb{A} \underline{\mathbf{v}}^{\prime} \times \mathbb{A} \underline{\mathbf{v}}^{\prime \prime}\right)$ disj $\subset \mathbb{A} \underline{\mathbf{v}}^{\prime} \times \mathbb{A} \underline{\mathbf{v}}^{\prime \prime}$ as before. More precisely we consider factorization induced from one for triangle and two-way parts. For triangle part, we compare eigenvalues of $B_{i}$ and $B_{i+1}^{\prime}$, hence second coordinates should be shifted as $w_{i+1, l}+\nu_{*}^{\mathbb{C}}$. (See (a)' in $\S 6.8 .2$ below.) We have factorization of $\mathcal{M}(\underline{\mathbf{v}}, \underline{\mathbf{w}})$ :

Theorem 6.9. $\mathcal{M}(\underline{\mathbf{v}}, \underline{\mathbf{w}})$ has a factorization morphism:

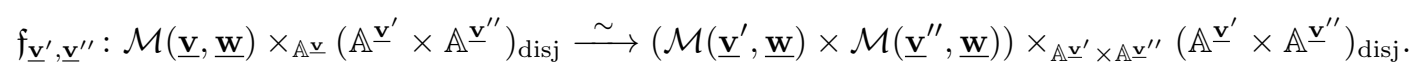

Corollary 6.10. The factorization morphism is compatible with the collapsing morphism in $\S 6.4$.

Consider coordinates $\left(w_{i, 1}, \ldots, w_{i, \mathbf{v}_{i}}, y_{i, 1}, \ldots, y_{i, \mathbf{v}_{i}}\right)$ in $\S 6.5$. Under the factorization, $w$-coordinates are grouped into two as $\left(w_{i, 1}, \ldots, w_{i, \mathbf{v}_{i}^{\prime}}\right),\left(w_{i, \mathbf{v}_{i}^{\prime}+1}, \ldots, w_{i, \mathbf{v}_{i}}\right)$. These can be considered as $w$ coordinates of $\mathcal{M}\left(\underline{\mathbf{v}}^{\prime}, \underline{\mathbf{w}}\right)$ and $\mathcal{M}\left(\underline{\mathbf{v}}^{\prime \prime}, \underline{\mathbf{w}}\right)$ respectively. Let $\left(y_{i, 1}^{\prime}, \ldots, y_{i, \mathbf{v}_{i}^{\prime}}^{\prime}\right),\left(y_{i, \mathbf{v}_{i}^{\prime}+1}^{\prime \prime}, \ldots, y_{i, \mathbf{v}_{i}}^{\prime}\right)$ be $y$-coordinates of $\mathcal{M}\left(\underline{\mathbf{v}}^{\prime}, \underline{\mathbf{w}}\right)$ and $\mathcal{M}\left(\underline{\mathbf{v}}^{\prime \prime}, \underline{\mathbf{w}}\right)$ respectively. Then we have

Lemma 6.11. The factorization morphism $\mathfrak{f}_{\underline{\mathbf{v}}^{\prime}, \underline{\mathbf{v}}^{\prime \prime}}$ sends $y_{i, k}$ to

$$
\begin{cases}y_{i, k}^{\prime} \prod_{l=\mathbf{v}_{i}^{\prime}+1}^{\mathbf{v}_{i}}\left(w_{i, k}-w_{i, l}\right) & \text { if } 1 \leq k \leq \mathbf{v}_{i}^{\prime}, \\ y_{i, k}^{\prime \prime} \prod_{l=1}^{\mathbf{v}_{i}^{\prime}}\left(w_{i, k}-w_{i, l}\right) & \text { if } \mathbf{v}_{i}^{\prime}+1 \leq k \leq \mathbf{v}_{i} .\end{cases}
$$

Proof. We have block decompositions $a_{i}=\left[\begin{array}{c}a_{i}^{\prime} \\ a_{i}^{\prime \prime}\end{array}\right], b_{i}=\left[\begin{array}{ll}b_{i}^{\prime} b_{i}^{\prime \prime}\end{array}\right], C_{\mathbf{w}_{i} \cdots 1, i}=\left[\begin{array}{cc}C_{\mathbf{w}_{i} \cdots 1, i}^{\prime} & 0 \\ 0 & C_{\mathbf{w}_{i} \cdots 1, i}^{\prime \prime}\end{array}\right]$, $B_{i}=\left[\begin{array}{cc}B_{i}^{\prime} & 0 \\ 0 & B_{i}^{\prime \prime}\end{array}\right]$. Therefore

$$
y_{i, k}=b_{i}^{\prime} \prod_{\substack{1 \leq l \leq \mathbf{v}_{i} \\ l \neq k}}\left(B_{i}^{\prime}-w_{i, l} \mathrm{id}\right) C_{\mathbf{w}_{i} \cdots 1, i}^{\prime} a_{i}^{\prime}+b_{i}^{\prime \prime} \prod_{\substack{1 \leq l \leq \mathbf{v}_{i} \\ l \neq k}}\left(B_{i}^{\prime \prime}-w_{i, l} \mathrm{id}\right) C_{\mathbf{w}_{i} \cdots 1, i}^{\prime \prime} a_{i}^{\prime \prime} .
$$

Suppose $1 \leq k \leq \mathbf{v}_{i}^{\prime}$. Then the second term vanishes as all eigenvalues of $B_{i}^{\prime \prime}$ appear in $w_{i, l}(l \neq k)$ with multiplicities.

Consider

$$
\prod_{\substack{1 \leq l \leq \mathbf{v}_{i} \\ l \neq k}}\left(B_{i}^{\prime}-w_{i, l} \text { id }\right)=\prod_{\substack{1 \leq l \leq \mathbf{v}_{i}^{\prime} \\ l \neq k}}\left(B_{i}^{\prime}-w_{i, l} \text { id }\right) \prod_{\mathbf{v}_{i}^{\prime}+1 \leq l \leq \mathbf{v}_{i}}\left(B_{i}^{\prime}-w_{i, l}\right) .
$$

Since $\prod_{1 \leq l \leq \mathbf{v}_{i}^{\prime}}\left(B_{i}^{\prime}-w_{i, l}\right.$ id $)=0$, we have

$$
\prod_{\substack{1 \leq l \leq \mathbf{v}_{i}^{\prime} \\ l \neq k}}\left(B_{i}^{\prime}-w_{i, l} \text { id }\right) B_{i}^{\prime}=\prod_{\substack{1 \leq l \leq \mathbf{v}_{i}^{\prime} \\ l \neq k}}\left(B_{i}^{\prime}-w_{i, l} \text { id }\right) w_{i, k} .
$$

Now the assertion is clear. The case $\mathbf{v}_{i}^{\prime}+1 \leq k \leq \mathbf{v}_{i}$ is the same.

Remark 6.12. This property for the case $\underline{\mathbf{w}}=0$ follows from the first part of the proof of [BDF14, Prop. 3.2], as it is a reduction to the SL(2)-case and works also for an affine case. The assertion for general $\underline{\mathbf{w}}$ follows from one for $\underline{\mathbf{w}}=0$ by Corollary 6.10 . We present a proof here for the sake of completeness.

\subsection{Normality}

Proposition 6.13. Over each point of $\mathbb{A}^{\mathbf{v}}$, the dimension of the fiber of $\Psi$ is $\sum\left(\mathbf{w}_{i}+1\right) \mathbf{v}_{i}^{2}+\mathbf{v}_{i}$.

Proof. The equations $B_{i}=D_{1, i} C_{1, i}$ and $C_{\mathbf{w}_{i}, i} D_{\mathbf{w}_{i}, i}=B_{i}^{\prime}$ mean $B_{i}$ and $B_{i}^{\prime}$ are always determined by a two-way part. Thus Propositions 3.10, 3.13 mean $\operatorname{dim} \Psi^{-1}(w)=\sum\left(\mathbf{w}_{i}+1\right) \mathbf{v}_{i}^{2}+\mathbf{v}_{i}$ for any $w \in \mathbb{A}$ ․ 
Corollary 6.14. $\mu^{-1}(0)$ is an irreducible reduced complete intersection in $\mathbb{M}$.

Theorem 6.15. $\mathcal{M}(\underline{\mathbf{v}}, \underline{\mathbf{w}})$ is normal.

Proof. We apply [CB03a, Cor. 7.2] in our case, i.e., we construct an open subscheme $U$ of $\mathcal{M}=$ $\mathcal{M}(\underline{\mathbf{v}}, \underline{\mathbf{w}})$ such that it is normal, the complement in $\mathcal{M}$ and the inverse image of the complement in $\widetilde{\mathcal{M}}$ both have codimension at least two, and $\widetilde{\mathcal{M}}$ has the property $\left(S_{2}\right)$ of Serre.

We consider the open subset $\AA \underline{\mathbf{v}} \subset \mathbb{A} \underline{\mathbf{v}}$ consisting of configurations where at most two points collide. (The same notation $\AA \underline{\mathbf{v}}$ will be used for a smaller open subset in the proof of Theorem 6.18 .) The complement of $\AA^{\mathbf{v}}$ is of codimension 2 , and the complements of $\Psi^{-1}\left(\AA^{\mathbb{\imath}} \underline{)}\right)$ in $\widetilde{\mathcal{M}}$ and $\Psi^{-1}\left(\AA^{\mathbb{v}}\right) / / \mathcal{G}$ in $\mathcal{M}(\underline{\mathbf{v}}, \underline{\mathbf{w}})$ respectively are of codimension 2 by Proposition 6.13. And $\widetilde{\mathcal{M}}$ is Cohen-Macaulay, hence has the property $\left(S_{2}\right)$.

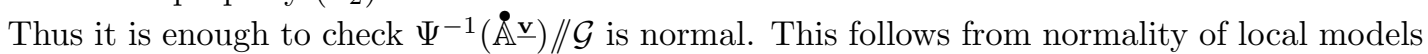
$\S 6.5 .1 \sim 6.5 .6$.

\subsection{Proof}

We now prove that the Coulomb branch $\mathcal{M}_{C}$ of a framed quiver gauge of an affine type $A_{n-1}$ is isomorphic to a bow variety in $§ 6.1$. We will use results in [BFN16a] frequently.

We consider a quiver gauge theory associated with two dimension vectors $\underline{\mathbf{v}}, \underline{\mathbf{w}}$. Namely we consider

$$
\mathbf{N}:=\bigoplus_{i=0}^{n-1} \operatorname{Hom}\left(\mathbb{C}^{\mathbf{v}_{i}}, \mathbb{C}^{\mathbf{v}_{i+1}}\right) \oplus \operatorname{Hom}\left(\mathbb{C}^{\mathbf{w}_{i}}, \mathbb{C}^{\mathbf{v}_{i}}\right)
$$

as a representation of $G:=\mathrm{GL}(\underline{\mathbf{v}})=\prod_{i} \mathrm{GL}\left(\mathbf{v}_{i}\right)$. Let $T$ be the product of maximal tori of $\operatorname{GL}\left(\mathbf{v}_{i}\right)$. Let $\mathbf{N}_{T}$ denote the restriction of $\mathbf{N}$ to $T$.

\subsection{1}

We first consider the case $\nu^{\mathbb{C}}=0$.

Let $\mathcal{O}=\mathbb{C}[[z]] \subset \mathcal{K}=\mathbb{C}((z))$. Let $\mathrm{Gr}_{G}$ denote the affine Grassmannian $G_{\mathcal{K}} / G_{\mathcal{O}}$. We consider the variety of triples $\mathcal{R}:=\left\{([g], s) \in \mathrm{Gr}_{G} \times \mathbf{N}_{\mathcal{O}} \mid g^{-1} s \in \mathbf{N}_{\mathcal{O}}\right\}$ and its equivariant Borel-Moore homology group $H_{*}^{G_{\mathcal{O}}}(\mathcal{R})$. See [BFN16a, $\left.\S 2\right]$. It is equipped with a convolution product, which is commutative. The Coulomb branch $\mathcal{M}_{C}$ is defined as its spectrum ([BFN16a, §3]). From its definition we have a morphism $\varpi: \mathcal{M}_{C} \rightarrow \operatorname{Spec} H_{G}^{*}(\mathrm{pt}) \cong \mathbb{A} \underline{\mathbf{v}}$. It is known that $\varpi^{-1}(\AA \mathbf{A} \underline{\mathbf{v}})$ is isomorphic to $\AA|\mathbf{v}| \times T^{\vee} / W$ so that $\varpi$ is the first projection ([BFN16a, Cor. 5.21]). Here $\AA|\underline{\mathbf{v}}|$ consists of distinct configurations (for each $i$ th and each pair $(i, i+1)$ ) as before, but we also require that 0 is not contained in a configuration for $i$ when $\mathbf{w}_{i} \neq 0$. In the terminology of [BFN16a, §5], $\AA|\mathbf{v}|$ is the complement of the union of all generalized root hyperplanes associated with $(G, \mathbf{N})$. Then we define $\AA$ 늠 as $\AA|\mathbf{v}| / \mathfrak{S}_{\mathbf{v}}$.

Let us consider $w_{i, k}$ as a class in $H_{\mathrm{GL}\left(\mathbf{v}_{i}\right)}^{2}(\mathrm{pt})$ corresponding to the $k$ th coordinate vector of $\mathbb{C}^{\mathbf{v}_{i}}$ so that the isomorphism $\operatorname{Spec} H_{G}^{*}(\mathrm{pt}) \cong \mathbb{A} \underline{\mathbf{v}}$ is explicit. We also take coordinates $\mathrm{u}_{i, k}$ of $T^{\vee}$, which arises as the fundamental class of the point $w_{i, k}^{*}$ in $\mathrm{Gr}_{T}$, the cocharacter of $T$ corresponding

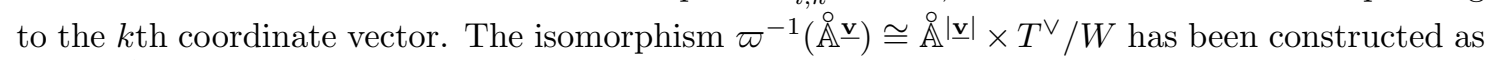
$\mathbf{z}^{*}\left(\iota_{*}\right)^{-1}$ where

$$
\iota_{*}: H_{*}^{T_{\mathcal{O}}}\left(\mathcal{R}_{T, \mathbf{N}_{T}}\right) \rightarrow H_{*}^{G_{\mathcal{O}}}(\mathcal{R}) \otimes_{H_{G}^{*}}(\mathrm{pt}) H_{T}^{*}(\mathrm{pt})
$$

is the pushforward homomorphism given by the inclusion $\mathcal{R}_{T, \mathbf{N}_{T}} \rightarrow \mathcal{R}$ from the variety of triples for $\left(T, \mathbf{N}_{T}\right)$ to one for $(G, \mathbf{N})$, and

$$
\mathbf{z}^{*}: H_{*}^{T_{\mathcal{O}}}\left(\mathcal{R}_{T, \mathbf{N}_{T}}\right) \rightarrow H_{*}^{T_{\mathcal{O}}}\left(\mathrm{Gr}_{T}\right)
$$

is the pull-back homomorphism induced from the inclusion $\mathbf{z}: \operatorname{Gr}_{T} \rightarrow \mathcal{R}_{T, \mathbf{N}_{T}}$ of the zero section ([BFN16a, §5]). Then $H_{*}^{T_{\mathcal{O}}}\left(\mathrm{Gr}_{T}\right) \cong \operatorname{Spec}\left(\mathbb{A}|\underline{\mathbf{v}}| \times T^{\vee}\right)([$ BFN16a, Prop.5.19]), and the isomorphism respects the Weyl group action. 
On the other hand, we have a morphism $\mathcal{M}(\underline{\mathbf{v}}, \underline{\mathbf{w}}) \rightarrow \mathbb{A} \underline{\mathbf{v}}$ induced from $\Psi$. Let us denote it also by $\Psi$ for brevity. By the factorization and models in $\S 6.5$, we have $\Psi^{-1}(\AA \underline{\mathbf{v}}) \cong \AA \mathbf{v} \times T^{\vee} / W$ : In fact, we first make a reduction to $\underline{\mathbf{v}}$ has 1 for an entry and 0 for others by the factorization. Then we have either 6.5.1 or 6.5.3. When $\mathbf{w}=0$, the model is $\mathbb{C}\left[w, y^{ \pm 1}\right]=\operatorname{Spec}\left(\mathbb{C} \times \mathbb{C}^{\times}\right)$. When $\mathbf{w} \neq 0$, the model is $\left\{x y=w^{\mathbf{w}}\right\}$, but our $\AA$ 兴 excludes 0, i.e., $w \neq 0$. Therefore $\left\{x y=w^{\mathbf{w}}, w \neq 0\right\} \cong \mathbb{C}^{\times} \times \mathbb{C}^{\times}$.

We define an isomorphism

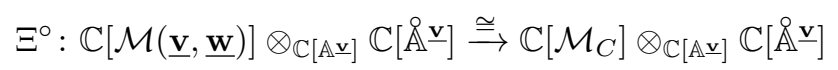

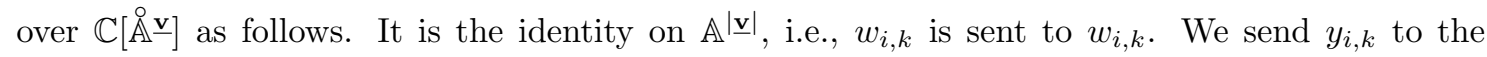
homology class $\iota_{*} \mathrm{y}_{i, k}$, where $\iota_{*}$ is as above, and

$$
\mathrm{y}_{i, k}=\left[\pi^{-1}\left(w_{i, k}^{*}\right)\right], \quad \pi: \mathcal{R}_{T, \mathbf{N}_{T}} \rightarrow \mathrm{Gr}_{T} ;([g], s) \mapsto[g] .
$$

Here $\left[\pi^{-1}\left(w_{i, k}^{*}\right)\right]$ denotes the fundamental class of $\pi^{-1}\left(w_{i, k}^{*}\right)$. By [BFN16a, $\S 4(v i)$ ] we have

$$
\mathbf{z}^{*}\left(\mathrm{y}_{i, k}\right)=\mathbf{u}_{i, k} \prod_{1 \leq l \leq \mathbf{v}_{i+1}}\left(w_{i+1, l}-w_{i, k}\right),
$$

if $n \geq 2$ (i.e., not a Jordan quiver) and

$$
\mathbf{z}^{*}\left(\mathrm{y}_{k}\right)=\mathbf{u}_{k} \prod_{\substack{1 \leq l \leq \mathbf{v} \\ l \neq k}}\left(w_{l}-w_{k}\right),
$$

if $n=1$. (We omit the subscript $i$ for Jordan quiver.) Therefore $\Xi^{\circ}$ is indeed a birational isomorphism. Moreover it is equivariant under Weyl group (i.e., $\mathfrak{S}_{\mathbf{v}}$ ) action, hence it indeed induces a birational isomorphism $\mathcal{M}_{C} \rightarrow \mathcal{M}(\underline{\mathbf{v}}, \underline{\mathbf{w}})$.

Theorem 6.18. $\Xi^{\circ}$ extends to an isomorphism $\Xi: \mathcal{M}_{C} \rightarrow \mathcal{M}(\underline{\mathbf{v}}, \underline{\mathbf{w}})$.

Proof. We can use [BFN16a, Th. 5.26], as we have proved that $\mathcal{M}(\underline{\mathbf{v}}, \underline{\mathbf{w}})$ is normal and all fibers of $\Psi$ have the same dimension. (See [BFN16a, Rem. 5.27].) The rest of the argument is exactly the same as one in [BFN16b, §3], but let us repeat it for completeness.

Let $\AA|\mathbf{v}|$ denote the complement of all pairwise intersections of generalized root hyperplanes as

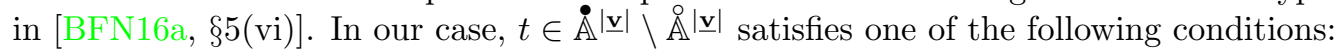

(a) $w_{i, k}(t)=w_{i+1, l}(t)$ for some $i, k, l$, but all others are distinct. Moreover $w_{j, r}(t) \neq 0$ if $\operatorname{dim} W_{j} \neq 0$.

(b) $w_{i, k}(t)=w_{i, l}(t)$ for distinct $k, l$ and some $i$, but all others are distinct. Moreover $w_{j, r}(t) \neq 0$ if $\operatorname{dim} W_{j} \neq 0$.

(c) All pairs like in (a),(b) are distinct, but $w_{i, k}(t)=0$ for $i$ with $W_{i} \neq 0$.

Let us assume $k=1$ and $l=1$ for (a), $l=2$ for (b) for brevity. Let $\left(G^{\prime}, \mathbf{N}^{\prime}\right)=\left(Z_{G}(t), \mathbf{N}^{t}\right)$. We have $\mathcal{R}_{G, \mathbf{N}}^{t}=\mathcal{R}_{Z_{G}(t), \mathbf{N}^{t}}$ by [BFN16a, Lem. 5.1]. Up to isomorphisms, we have $\left(G^{\prime}, \mathbf{N}^{\prime}\right)=$ $\left(\mathrm{GL}\left(\mathbf{v}^{\prime}\right) \times T^{\left|\underline{\mathbf{v}}^{\prime \prime}\right|}, \mathbf{N}\left(\underline{\mathbf{v}}^{\prime}, \underline{\mathbf{w}}^{\prime}\right)\right)$ where $\mathbf{N}\left(\underline{\mathbf{v}}^{\prime}, \underline{\mathbf{w}}^{\prime}\right)$ is the vector space associated with dimension vectors $\underline{\mathbf{v}}^{\prime}, \underline{\mathbf{w}}^{\prime}$ given below, and $\underline{\mathbf{v}}^{\prime \prime}=\underline{\mathbf{v}}-\underline{\mathbf{v}}^{\prime}$. Here $T^{\left|\underline{\mathbf{v}}^{\prime \prime}\right|}$ acts trivially on $\mathbf{N}^{\prime}=\mathbf{N}\left(\underline{\mathbf{v}}^{\prime}, \underline{\mathbf{w}}^{\prime}\right)$.

In case $(\mathrm{a}), \underline{\mathbf{w}}^{\prime}=0, \mathbf{v}_{i}^{\prime}=1=\mathbf{v}_{i+1}^{\prime}$ and other entries are 0 . (We understand $i \neq i+1$, in particular $n \geq 2$. The case $n=1$ is treated in case (b).)

In case $(\mathrm{b}), \underline{\mathbf{w}}^{\prime}=0, \mathbf{v}_{i}^{\prime}=2$ and other entries are 0 .

In case $(\mathrm{c}), \mathbf{v}_{i}^{\prime}=1, \mathbf{w}_{i}^{\prime}=\mathbf{w}_{i}$ and other entries are 0 .

Let us consider the Coulomb branch $\mathcal{M}_{C}\left(G^{\prime}, \mathbf{N}^{\prime}\right)$ of $\left(G^{\prime}, \mathbf{N}^{\prime}\right)$. It has an isomorphism

$$
\mathbf{z}^{\prime *}\left(\iota_{*}^{\prime}\right)^{-1}: \mathbb{C}\left[\mathcal{M}_{C}\left(G^{\prime}, \mathbf{N}^{\prime}\right)\right] \otimes_{H_{G^{\prime}}^{*}(\mathrm{pt})} \mathbb{C}[\AA|\mathbf{v}|] \cong \mathbb{C}\left[\AA|\mathbf{v}| \times T^{\vee}\right]
$$


in the same way as $\mathcal{M}$. Here $\mathbf{z}^{\prime}, \iota^{\prime}$ are morphisms $\mathbf{z}, \iota$ as above for $\left(G^{\prime}, \mathbf{N}^{\prime}\right)$. Therefore we have an isomorphism

$$
\Xi^{t \circ}: \mathbb{C}[\mathcal{M}(\underline{\mathbf{v}}, \underline{\mathbf{w}})] \times_{\mathbb{C}[\mathbb{A} \mathbf{v}]} \mathbb{C}\left[\stackrel{\AA}{\mathbb{A}}|\underline{\mathbf{v}}| \stackrel{\cong}{\cong} \mathbb{C}\left[\mathcal{M}_{C}\left(G^{\prime}, \mathbf{N}^{\prime}\right)\right] \otimes_{H_{G^{\prime}}^{*}(\mathrm{pt})} \mathbb{C}[\stackrel{\AA}{\mathbb{A}}|\underline{\mathbf{v}}|]\right.
$$

as the composite $\iota_{*}^{\prime} \mathbf{z}^{\prime *-1} \mathbf{z}^{*}\left(\iota_{*}\right)^{-1} \Xi^{\circ}$. If we check that $\Xi^{t \circ}$ extends to an isomorphism $\Xi^{t}$ over

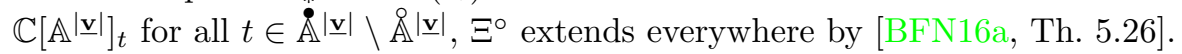

In order to the check this assertion, we first replace the left hand side of (6.19). We have a factorization morphism

$$
\mathfrak{f}_{\underline{\mathbf{v}}^{\prime}, \underline{\mathbf{v}}^{\prime \prime}}: \mathcal{M}(\underline{\mathbf{v}}, \underline{\mathbf{w}}) \times \mathbb{A} \underline{\mathbf{v}}\left(\mathbb{A} \underline{\mathbf{v}}^{\prime} \times \mathbb{A}^{\left|\underline{\mathbf{v}}^{\prime \prime}\right|}\right) \rightarrow \mathcal{M}\left(\underline{\mathbf{v}}^{\prime}, \underline{\mathbf{w}}\right) \times T^{*}\left(T^{\left|\underline{\mathbf{v}}^{\prime \prime}\right|}\right)^{\vee}
$$

defined in an appropriate open subvariety. To study $\Xi^{t \circ}$ at $t$ as above, we can further replace the right hand side by $\mathcal{M}\left(\underline{\mathbf{v}}^{\prime}, \underline{\mathbf{w}}^{\prime}\right) \times T^{*}\left(T^{\left|\underline{\mathbf{v}}^{\prime \prime}\right|}\right)^{\vee}$, as $\mathcal{M}\left(\underline{\mathbf{v}}^{\prime}, \underline{\mathbf{w}}^{\prime}\right)$ is an open subset in $\mathcal{M}\left(\underline{\mathbf{v}}^{\prime}, \underline{\mathbf{w}}\right)$ containing relevant points.

On the other hand, consider the right hand side of (6.19). Since $G^{\prime}=\mathrm{GL}\left(\underline{\mathbf{v}}^{\prime}\right) \times T \underline{\mathbf{v}}^{\prime \prime} \mid$ where the second factor acts trivially on $\mathbf{N}^{\prime}$, we have $\mathcal{M}\left(G^{\prime}, \mathbf{N}^{\prime}\right) \cong \mathcal{M}\left(G L\left(\underline{\mathbf{v}}^{\prime}\right), \mathbf{N}^{\prime}\right) \times T^{*}\left(T^{\left|\mathbf{v}^{\prime \prime}\right|}\right)^{\vee}([\mathrm{BFN} 16 \mathrm{a}$, Prop. 5.19]). Considering $\left(\mathrm{GL}\left(\underline{\mathbf{v}}^{\prime}\right), \mathbf{N}^{\prime}\right)$ as a special case of $(G, \mathbf{N})$, we can define

$$
\Xi^{\prime \circ}: \mathbb{C}\left[\mathcal{M}\left(\underline{\mathbf{v}}^{\prime}, \underline{\mathbf{w}}^{\prime}\right)\right] \otimes_{\mathbb{C}\left[\mathbb{A} \underline{\mathbf{w}}^{\prime}\right]} \mathbb{C}\left[\AA \underline{\mathbb{N}}^{\prime}\right] \stackrel{\cong}{\longrightarrow}\left[\mathcal{M}_{C}\left(\mathrm{GL}\left(\underline{\mathbf{v}}^{\prime}\right), \mathbf{N}^{\prime}\right)\right] \otimes_{\mathbb{C}\left[\mathbb{A} \underline{\mathbf{v}}^{\prime}\right]} \mathbb{C}\left[\AA \underline{\mathbb{A}}^{\prime}\right] .
$$

If we denote $y$-coordinates of $\mathcal{M}\left(\underline{\mathbf{v}}^{\prime}, \underline{\mathbf{w}}^{\prime}\right)$ by $y_{j, r}^{\prime}$, and the corresponding homology class by $\mathrm{y}_{j, r}^{\prime}, \Xi^{\prime \circ}$ sends $y_{j, r}^{\prime}$ to $\iota_{*}^{\prime} \mathrm{y}_{j, r}^{\prime}$. By Lemma $6.11, \mathfrak{f}_{\mathbf{v}^{\prime}, \mathbf{v}^{\prime \prime}}^{*}\left(y_{j, r}^{\prime}\right)$ and $y_{j, r}$ differ by a factor of a regular function which does not vanish at $t$. Also (6.16) in this case implies

$$
\mathbf{z}^{\prime *}\left(\mathrm{y}_{j, r}^{\prime}\right)=\mathrm{u}_{j, r} \prod_{1 \leq s \leq \mathbf{v}_{j+1}^{\prime}}\left(w_{j+1, s}-w_{j, r}\right)=\mathbf{z}^{*}\left(\mathrm{y}_{j, r}\right) \prod_{\mathbf{v}_{j+1}^{\prime}+1 \leq s \leq \mathbf{v}_{j+1}}\left(w_{j+1, s}-w_{j, r}\right)^{-1}
$$

Similar formula is true for (6.17). Thus $\mathbf{z}^{\prime *}\left(\mathrm{y}_{j, r}^{\prime}\right)$ and $\mathbf{z}^{*}\left(\mathrm{y}_{j, r}\right)$ also differ only by a regular function which does not vanish at $t$. Therefore $\Xi^{t \circ}$ extends across $t$ if and only if $\Xi^{\prime 0}$ does. Namely it is enough to show the assertion for cases (a),(b),(c) above.

In case $(\mathrm{a}), \mathcal{M}\left(\underline{\mathbf{v}}^{\prime}, \underline{\mathbf{w}}^{\prime}\right)$ is described in 6.5.4 $(n=2)$ and 6.5.6 $(n \geq 3)$. The corresponding Coulomb branch $\mathcal{M}_{C}\left(\overline{\mathrm{GL}}\left(\underline{\mathbf{v}}^{\prime}\right), \mathbf{N}^{\prime}\right)$ is described in [BFN16a, Th. 4.1]. If we identify $y_{12}$ in 6.5.4, 6.5.6 with the fundamental class of the fiber over the point $-w_{i, k}^{*}-w_{i+1, l}^{*}$, we can check that $\Xi^{\prime \circ}$ extends to an isomorphism $\mathcal{M}_{C}\left(\mathrm{GL}\left(\underline{\mathbf{v}}^{\prime}\right), \mathbf{N}^{\prime}\right) \rightarrow \mathcal{M}\left(\underline{\mathbf{v}}^{\prime}, \underline{\mathbf{w}}^{\prime}\right)$.

Case (c) is similar. $\mathcal{M}\left(\underline{\mathbf{v}}^{\prime}, \underline{\mathbf{w}}^{\prime}\right)$ is described in 6.5.1 $(n=1)$ and 6.5.3 $(n \geq 2)$. The corresponding Coulomb branch is described again in [BFN16a, Th 4.1]. If we identify $x$ in 6.5.1, 6.5.3 with the fundamental class of the fiber over $-w_{i, k}^{*}$, we can check that $\Xi^{\prime \circ}$ extends.

In case (b), $\mathcal{M}\left(\underline{\mathbf{v}}^{\prime}, \underline{\mathbf{w}}^{\prime}\right)$ is described in 6.5.2 $(n=1)$ and 6.5.5 $(n \geq 2)$. When $n=1$ (i.e., Jordan quiver $), \mathcal{M}\left(\underline{\mathbf{v}}^{\prime}, \underline{\mathbf{w}}^{\prime}\right) \cong S^{2}\left(\mathbb{C} \times \mathbb{C}^{\times}\right)$given by $\left(w_{1}, w_{2}, y_{1}, y_{2}\right) / \mathfrak{S}_{2}$. This is the same as the description of the Coulomb branch in [BFN16a, Prop. 6.14]. If $n \geq 2$, we have GL( $\left.\underline{\mathbf{v}}^{\prime}\right)=\mathrm{GL}(2), \mathbf{N}^{\prime}=0$. The corresponding Coulomb branch, more precisely its ramified $\mathfrak{S}_{2}$-cover, can be identified with $\mathbb{C}\left[w_{1}, w_{2}, y_{1}^{ \pm}, y_{2}^{ \pm}, \xi\right] /\left(y_{1}-y_{2}-\xi\left(w_{1}-w_{2}\right)\right]$ in 6.5 .5 , where $\xi$ is the fundamental class of the closed $\mathrm{GL}(2)_{\mathcal{O}}$-orbit through cocharacters $w_{1}^{*}$ and $w_{2}^{*}$, which is isomorphic to $\mathbb{P}^{1}$. Moreover $y_{1}^{-1}, y_{2}^{-1}$ are fundamental classes of points $-w_{1}^{*},-w_{2}^{*}$. The computation is similar to [BFN16a, Lem. 6.9], hence the further detail is omitted.

\subsection{2}

Next consider the case with parameter $\nu^{\mathbb{C}}$. It corresponds to gauge theories with flavor symmetries. We consider the standard action of $T^{\mathbf{w}_{i}}$ on $\mathbb{C}^{\mathbf{w}_{i}}$, and the induced action of $T(\underline{\mathbf{w}}):=\prod_{i} T^{\mathbf{w}_{i}}$ on $\mathbf{N}$. Note that the action of scalars in $T(\underline{\mathbf{w}})$ is same as that of scalars in $G$. Hence we have the induced action of $(G \times T(\underline{\mathbf{w}})) / \mathbb{C}^{\times}$. We also have the $\mathbb{C}^{\times}$-action on the component $\bigoplus_{i=0}^{n-1} \operatorname{Hom}\left(\mathbb{C}^{\mathbf{v}_{i}}, \mathbb{C}^{\mathbf{v}_{i+1}}\right)$ of $\mathbf{N}$ by scaling. The latter is defined also for $\underline{\mathbf{w}}=0$. Let $\tilde{G}=(G \times T(\underline{\mathbf{w}})) / \mathbb{C}^{\times} \times \mathbb{C}^{\times}$when $\underline{\mathbf{w}} \neq 0$, and $G \times \mathbb{C}^{\times}$when $\underline{\mathbf{w}}=0$. We have the induced action of $\tilde{G}_{\mathcal{O}}$ on $\mathcal{R}$, and consider a larger 
equivariant Borel-Moore homology group $H_{*}^{\tilde{G}_{\mathcal{O}}}(\mathcal{R})$. It is equipped with a convolution product, which is commutative. We have a morphism $\varpi: \operatorname{Spec} H_{*}^{\tilde{G}_{\mathcal{O}}}(\mathcal{R}) \rightarrow \operatorname{Spec} H_{\tilde{G}}^{*}(\mathrm{pt}) \cong \mathbb{A}^{\mathbf{v}} \times \mathbb{A}^{|\underline{\mathbf{w}}|}$ or $\mathbb{A} \mathbf{v} \times \mathbb{A}$. We denote additional coordinates by $\nu_{k, i}^{\mathbb{C}}\left(i=0, \ldots, n-1, k=1, \ldots, \mathbf{w}_{i}\right), \nu_{*}^{\mathbb{C}}$ when $\mathbf{w} \neq 0$ and $\nu_{*}^{\mathbb{C}}$ when $\mathbf{w}=0$. These are identified with parameters $\nu_{k, i}^{\mathbb{C}}, \nu_{*}^{\mathbb{C}}$ of bow varieties.

We define $\AA|\underline{\mathbf{v}}|+|\underline{\mathbf{w}}|, \AA|\mathbf{v}|+|\underline{\mathbf{w}}|$ and $\AA|\underline{\mathbf{v}}|+1, \AA^{\mid \mathbf{v}} \mid+1$ as above, but we consider differences $w_{i+1, l}(t)+$ $\nu_{*}^{\mathbb{C}}(t)-w_{i, k}(t), w_{i, k}(t)-w_{i, l}(t), w_{i, r}(t)-\nu_{j, p}^{\mathbb{C}}(t)$, and conditions are

(a)' $w_{i+1, l}(t)-w_{i, k}(t)+\nu_{*}^{\mathbb{C}}(t)=0$ for some $i, k, l$, but all others are differences are nonzero. $(n=1$ case is included unlike undeformed case.)

(b)' $w_{i, k}(t)-w_{i, l}(t)=0$ for distinct $k, l$ and some $i$, but all other differences are nonzero.

(c)' $w_{i, k}(t)-\nu_{i, p}^{\mathbb{C}}(t)+\nu_{*}^{\mathbb{C}}(t)=0$ for some $i, k, p$, but all other differences like in (a)',(b)' are nonzero.

We define $\Xi^{\circ}$ over $\AA|\underline{\mathbf{v}}|+|\underline{\mathbf{w}}|$ or $\AA|\underline{\mathbf{v}}|+1$ as above, and check that $\Xi^{\text {to }}$ extends for $t \in \AA|\mathbf{v}|+|\underline{\mathbf{w}}| \mid$ $\AA|\underline{\mathbf{v}}|+|\underline{\mathbf{w}}|$ or $\AA|\underline{\mathbf{v}}|+1 \backslash \AA|\mathbf{v}|+1$. It is reduced to checks for local models as above. For (c)', we use [BFN16a, §4(iii)].

For (a)', let us consider $\mathbf{N}^{t}$. It is the space for finite type $A_{2}$ gauge theory with $\underline{\mathbf{v}}=(1,1)$. Note that endomorphisms coming back from $w_{i+1, l}^{*}$ to $w_{i, k}^{*}$ (when $n=2$ ) is not fixed by $t$, as the difference is $\nu_{*}^{\mathbb{C}}(t)$, not $-\nu_{*}^{\mathbb{C}}(t)$. Similary we do not have coming back endomorphisms even for $n=1$. By the same reason, the factorization of $\mathcal{M}_{\nu_{*}^{\complement}}(\underline{\mathbf{v}}, 0)$ yields a finite type $A_{2}$ bow variety. For example, only one of either $w_{1}-w_{2}+\nu_{*}^{\mathbb{C}}$ or $w_{1}-w_{2}-\nu_{*}^{\mathbb{C}}$ vanishes in 6.5.4. Therefore the local model which we should use is one in 6.5.6 with $\underline{\mathbf{w}}=0$.

For (b)', we have $\left(G^{\prime}, \mathbf{N}^{\prime}\right)=\left(\operatorname{GL}(2) \times T^{\left|\mathbf{v}^{\prime}\right|}, \overline{0}\right)$. This is clear for $n \geq 2$, but it is also true for $n=1$, as $\operatorname{Hom}\left(\mathbb{C}^{\mathbf{v}_{i}}, \mathbb{C}^{\mathbf{v}_{i+1}}\right)=\operatorname{Hom}\left(\mathbb{C}^{\mathbf{v}_{0}}, \mathbb{C}^{\mathbf{v}_{0}}\right)$ is multiplied by scalar corresponding to $\nu_{*}^{\mathbb{C}}$. Similarly the factorization implies $A=0$ in the relevant summand even for $n=1$, hence the local model is 6.5.5 with $\mathbf{w}=0$.

The remaining argument is the same as the $\nu^{\mathbb{C}}=0$ case.

\subsection{Identifications of additional structures}

\subsubsection{Poisson structures}

Recall $H_{*}^{G_{\mathcal{O}}}(\mathcal{R})$ carries a Poisson structure from the noncommutative deformation $H_{*}^{G_{\mathcal{O}} \rtimes \mathbb{C}^{\times}}(\mathcal{R})$ ([BFN16a, $\S 3(\mathrm{iv})])$. On the other hand, $\mathcal{M}(\underline{\mathbf{v}}, \underline{\mathbf{w}})$ has the Poisson structure compatible with the symplectic structure on $\mathcal{M}^{\mathrm{s}}(\underline{\mathbf{v}}, \underline{\mathbf{w}})$, as a reduction.

Proposition 6.20. The isomorphism $\Xi$ in Theorem 6.18 respects the Poisson structure if we multiply -1 on the bracket for the bow variety.

Proof. It is enough to check that the symplectic form is respected over $\AA|\mathbf{v}|$. Moreover we can replace $\mathcal{M}(\underline{\mathbf{v}}, \underline{\mathbf{w}})$ by the chainsaw quiver variety $\mathcal{M}(\underline{\mathbf{v}}, 0)$ thanks to Proposition 6.8 .

We compare Poisson brackets among $\mathrm{y}_{i, k}, w_{j, l}$ and $y_{i, k}, w_{j, l}$. We have $\left\{w_{i, k}, w_{j, l}\right\}=0$ by [BFN16a, $\S 3($ vi)] for the Coulomb branch, and by Proposition 5.8 for the bow variety.

Let us first suppose $n \neq 1$. By [BFN16a, Cor. 5.21(2)] we have $\left\{\mathbf{u}_{i, k}, w_{j, l}\right\}=\delta_{i j} \delta_{k l} \mathbf{u}_{i, k}$ and other brackets are 0 . Therefore

$$
\begin{gathered}
\left\{\mathrm{y}_{i, k}, w_{j, l}\right\}=\delta_{i j} \delta_{k l} \mathrm{y}_{i, k}, \quad\left\{\mathrm{y}_{i, k}, \mathrm{y}_{j, l}\right\}=0 \quad \text { if }|i-j| \neq 1, \\
\left\{\mathrm{y}_{i, k}, \mathrm{y}_{i+1, l}\right\}=\frac{\mathrm{y}_{i, k} \mathrm{y}_{i+1, l}}{w_{i, k}-w_{i+1, l}}
\end{gathered}
$$

by (6.16). Here we assume $n \geq 3$. For $n=2$, there is an extra contribution as $i+2 \equiv i$ modulo 2. We have

$$
\left\{\mathrm{y}_{i, k}, \mathrm{y}_{i+1, l}\right\}=\frac{2 \mathrm{y}_{i, k} \mathrm{y}_{i+1, l}}{w_{i, k}-w_{i+1, l}}
$$


in the last equality. The coefficient 0,1 or 2 for $\left\{\mathrm{y}_{i, k}, \mathrm{y}_{j, l}\right\}$ is uniformly written as $2 \delta_{i j}-c_{i j}$ by the Cartan matrix $\left(c_{i j}\right)$. Comparing it with [FR14, $\left.(24),(25)\right]$, we find this Poisson bracket is negative of one for the chainsaw quiver variety. (The indices $i, k$ are swapped, and our $w_{i, k}$ (resp. $y_{i, k}$ ) is $x_{k, i}\left(\right.$ resp. $\left.y_{k, i}\right)$ in [FR14]. Also the sign for [FR14, (25)] is wrong, and missed in the last displayed formula in the proof. Compare it with [FR14, Prop. 3.5].)

Next suppose $n=1$. By (6.17) we have

$$
\left\{\mathrm{y}_{k}, w_{l}\right\}=\delta_{k l} \mathrm{y}_{k}, \quad\left\{\mathrm{y}_{k}, \mathrm{y}_{l}\right\}=\frac{2\left(1-\delta_{k l}\right) \mathrm{y}_{k} \mathrm{y}_{l}}{w_{k}-w_{l}} .
$$

On the other hand, $\mathcal{M}(\mathbf{v}, 0)$ is the reduction of $\widetilde{\mathcal{M}}$ in $\S 3.1$ (triangle) by the diagonal $\mathrm{GL}(V) \subset$ $\mathrm{GL}(V) \times \mathrm{GL}(V)$, where we take $V_{1}=V_{2}=V$. We calculate the Poisson bracket as in [FR14, $(24),(25)]$ to deduce the same assertion. We have 2 in the coefficient, as both $p_{k, i}$ and $q_{k, i}$ contribute in contrast to the calculation in [FR14].

\subsubsection{Hamiltonian torus action}

Recall $\mathcal{R}$ has connected components parametrized by $\pi_{1}\left(\mathrm{Gr}_{G}\right)$, and hence $H_{*}^{G_{\mathcal{O}}}(\mathcal{R})$ is graded by $\pi_{1}(G)$ ([BFN16a, $\left.\S 3(\mathrm{v})\right]$ ). Therefore $\mathcal{M}_{C}$ has an action of $\pi_{1}(G)^{\vee}$, where $\pi_{1}(G)^{\vee}$ is the Pontryagin dual of $\pi_{1}(G)$. Since $G=\prod_{i} \mathrm{GL}\left(\mathbf{v}_{i}\right), \pi_{1}(G)^{\vee}=\left(\mathbb{Z}^{n}\right)^{\vee}$ is the $n$-dimensional torus $\left(\mathbb{C}^{\times}\right)^{n}$. Let us denote coordinates as $\left(s_{0}, s_{1}, \ldots, s_{n-1}\right)$ so that the $i$-th vertex gives $s_{i}$.

On the other hand, the bow variety $\mathcal{M}(\underline{\mathbf{v}}, \underline{\mathbf{w}})$ has the action of the $n$-dimensional torus by scalar multiplication on $\mathbb{C}_{x}$ for each $x \in \Lambda$. Using the numbering on $x$, we have coordinates $\left(t_{0}, t_{1}, \ldots, t_{n-1}\right)$ on the torus. Note that the action of the 1-dimensional diagonal subgroup $(t, t, \ldots, t)$ can be absorbed to $\prod \mathrm{GL}\left(V_{\zeta}\right)$, hence is trivial on the quotient space $\mathcal{M}(\underline{\mathbf{v}}, \underline{\mathbf{w}})$.

We have an extra $\mathbb{C}^{\times}$-action given by $(A, b) \mapsto\left(t_{\delta} A, t_{\delta} b\right)$ at the vertex 0 , and other entries are unchanged. This action can be absorbed to $\prod \mathrm{GL}\left(V_{\zeta}\right)$ if some $V_{\zeta}=0$, but cannot in general. It corresponds to the central extension of the affine Lie algebra.

Let us choose an isomorphism $\left\{\left(t_{0}, \ldots, t_{n-1}, t_{\delta}\right)\right\} /\{(t, t, \ldots, t, 1)\} \cong\left(\mathbb{C}^{\times}\right)^{n}$ by $s_{i}=t_{i+1} t_{i}^{-1}$ for $i \neq n-1, s_{n-1}=t_{0} t_{n-1}^{-1} t_{\delta}$.

Proposition 6.21. The isomorphism $\Xi$ in Theorem 6.18 respects the $\left(\mathbb{C}^{\times}\right)^{n}$-action.

Proof. It is enough to check the assertion over $\AA|\mathbf{v}|$.

Let us take $s=\left(s_{i}\right) \in\left(\mathbb{C}^{\times}\right)^{n}$. Since $w_{i, k}$ is an equivariant variable, $s \cdot w_{i, k}=w_{i, k}$ in the Coulomb branch. On the other hand, $w_{i, k}$ is an eigenvalue of $B_{i}$, we also have $s \cdot w_{i, k}=w_{i, k}$ in the bow variety.

Since $\mathrm{y}_{i, k}$ is the fundamental class of $\pi^{-1}\left(w_{i, k}^{*}\right), s \cdot \mathrm{y}_{i, k}=s_{i} \mathrm{y}_{i, k}$. On the other hand, by the definition of $y_{i, k}$ we have $t \cdot y_{i, k}=t_{i+1} t_{i}^{-1} y_{i, k}$ if $i \neq n-1$ and $t \cdot y_{n-1, k}=t_{0} t_{n-1}^{-1} t_{\delta} y_{n-1, k}$. Now the assertion is checked.

Note that the $\left(\mathbb{C}^{\times}\right)^{n}$-action preserves the symplectic form, which can be checked easily in either point of view.

\subsection{3 $\mathbb{C}^{\times}$-action}

Let $\operatorname{deg}_{h}$ denote the cohomological degree for the Coulomb branch $H_{*}^{G_{\mathcal{O}}}(\mathcal{R})=\mathbb{C}[\mathcal{M}]$. Since $w_{i, k}$ is a class in $H_{\mathrm{GL}\left(\mathbf{v}_{i}\right)}^{2}(\mathrm{pt})$, we have $\operatorname{deg}_{h} w_{i, k}=2$. On the other hand, $\mathrm{y}_{i, k}$ is the fundamental class of $\pi^{-1}\left(w_{i, k}^{*}\right)$. This fiber is an infinite dimensional vector space, but its degree is defined as a codimension in relative to another infinite dimensional vector space, the fiber of $\mathcal{T}$ in [BFN16a, $\S 2]$. The codimension is given by the formula in [BFN16a, Lem. 2.2]. We have

$$
\operatorname{deg}_{h} \mathrm{y}_{i, k}=2 d_{w_{i, k}^{*}}=2 \sum_{\chi} \max \left(-\left\langle\chi, w_{i, k}^{*}\right\rangle, 0\right) \operatorname{dim} \mathbf{N}(\chi)=2 \mathbf{v}_{i+1},
$$


where $\mathbf{N}(\chi)$ is the weight space of $\mathbf{N}$ with weight $\chi$. The cohomological degree must be corrected in the monopole formula, as noted in [BFN16a, Rem. 2.8(2)]. The correction term is

$$
\sum\left\langle\chi, w_{i, k}^{*}\right\rangle \operatorname{dim} \mathbf{N}(\chi)=-\mathbf{v}_{i+1}+\mathbf{v}_{i-1}+\mathbf{w}_{i} .
$$

Let $\operatorname{deg}_{m}$ denote the corrected degree in the monopole formula. We thus have

$$
\operatorname{deg}_{m} w_{i, k}=2, \quad \operatorname{deg}_{m} \mathrm{y}_{i, k}=\mathbf{v}_{i+1}+\mathbf{v}_{i-1}+\mathbf{w}_{i} .
$$

We have the corresponding $\mathbb{C}^{\times}$-action on $\mathcal{M}$ so that $\operatorname{deg}_{m}$ gives weight.

A general expectation is that the corresponding $S^{1}$-action, the restriction of the $\mathbb{C}^{\times}$-action, extends to an $\mathrm{SU}(2)$-action on $\mathcal{M}$ which rotates the complex structures. (See [BFN16a, Rem. 2.8(2)].)

Let us construct an $\mathrm{SU}(2)$-action on $\mathcal{M}$. We use the original definition of $\mathcal{M}$ in $\S 2.1$. For $\left(B^{L R}, B^{R L}\right)$, we define the quaternion module structure by $J\left(B^{L R}, B^{R L}\right)=\left(-\left(B^{R L}\right)^{\dagger},\left(B^{L R}\right)^{\dagger}\right)$, and consider the induced $\mathrm{SU}(2)=\mathrm{Sp}(1)$-action. Here ${ }^{\dagger}$ denote the hermitian adjoint. The $S^{1}-$ action has weight 1 on both $B^{L R}, B^{R L}$. The same construction applies to $I, J$.

Let $\left(\nabla, T_{1}, T_{2}, T_{3}\right)$ be a solution of Nahm's equations on an interval with a $x$-point in the middle, corresponding to the triangle part. We consider $T:=T_{1} i+T_{2} j+T_{3} k$ as an imaginary quaternion valued function, and consider the adjoint $\operatorname{Sp}(1)$-action $q T q^{-1}(q \in \operatorname{Sp}(1))$. This action does not preserve the condition (b) in $\S 2.1$ as $\left(\rho_{1}, \rho_{2}, \rho_{3}\right)$ is also changed by the adjoint action. Therefore we compose the gauge transformation which is $\rho(q)^{-1}$ on $\left(\left.E_{\zeta^{ \pm}}\right|_{x}\right)^{\perp}$ at $x$. Here $\rho$ is a homomorphism $\mathrm{Sp}(1) \rightarrow \mathrm{U}(\Delta R(x))$ associated with $\left(\rho_{1}, \rho_{2}, \rho_{3}\right)$.

Let us restrict to the $S^{1}$-action. The first adjoint action is restricted to weight 2 -action on $T_{2}+T_{3} i$. Since $\eta$ in Proposition 3.2 is the value of $T_{2}+T_{3} i$ at a certain point, it has weight 2 . On the other hand, the second action is restricted to the conjugation by

$$
\operatorname{diag}(\underbrace{1, \ldots, 1}_{m}, t^{n-m-1}, t^{n-m-3}, \ldots, t^{1-n+m}) \quad t \in S^{1} .
$$

Therefore in sum, weights on $\left(A, B_{1}, B_{2}, a, b\right)$ of the triangle part are

$$
\mathrm{wt}\left(A, B_{1}, B_{2}, a, b\right)=\left(0,2,2,1+\mathbf{v}_{1}-\mathbf{v}_{2}, 1+\mathbf{v}_{2}-\mathbf{v}_{1}\right) .
$$

From the definition of $w_{i, k}, y_{i, k}$, we have

$$
\text { wt } w_{i, k}=2, \quad \text { wt } y_{i, k}=\mathbf{v}_{i-1}+\mathbf{v}_{i+1}+\mathbf{w}_{i} \text {. }
$$

Thus

Proposition 6.22. The $\mathbb{C}^{\times}$-action on $\mathcal{M}$ given by the corrected degree is coming from the $\mathrm{SU}(2)$ action defined as above.

\section{Hanany-Witten transition and its applications}

As explained in $\S 2$, a bow diagram comes from a brane configuration in type IIB string theory [HW97]. It was observed that a new 3-brane is created when a D5- and an NS5-brane pass through each other. This is called Hanany-Witten transition. We interpret it as an isomorphism of two bow varieties and study its applications in this section.

\section{1 $3 d$ Mirror symmetry}

Before explaing Hanany-Witten transition, we review the explanation of the $3 d$ mirror symmetry via $\mathrm{SL}(2, \mathbb{Z})$-symmetry of the string theory, as a warming up example.

In order to obtain a bow diagram from a brane configuration, it is enough to replace a Dirichlet 5-brane with $\times$, and a NS 5-brane with 0 . And by exchanging NS 5-branes and Dirichlet 5-branes in a brane configuration, we get the dual theory $B$ of the original theory $A$, which is given by 
the original brane configuration. In physics, it is known that it gives $3 d$ mirror symmetry, hence the Coulomb branch of theory $A$ coincides with the Higgs branch of theory $B$ as holomorphic symplectic manifolds:

$$
\mathcal{M}_{C}^{A} \cong \mathcal{M}_{H}^{B}, \quad \mathcal{M}_{H}^{A} \cong \mathcal{M}_{C}^{B}
$$

By using these relations, we can easily get the Coulomb branch from the Higgs branch of the same theory:

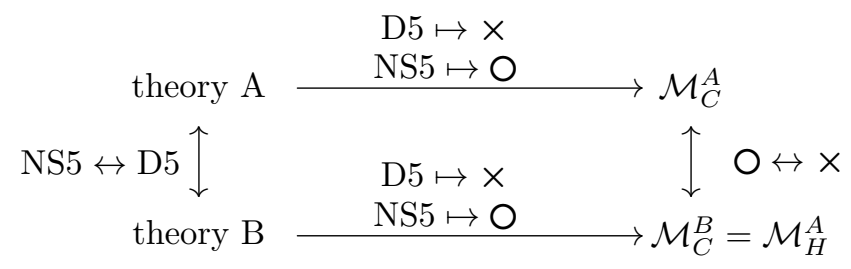

In the quiver gauge theory, a Higgs branch is given as a quiver variety $\mathfrak{M}_{0}(\underline{\mathbf{v}}, \underline{\mathbf{w}})$, so we can get the corresponding Coulomb branch as the bow variety which is given by the following procedure: (i) Rewrite a quiver variety as a bow variety associated with a bow diagram with cobalanced dimension vector (see Theorem 2.15).

(ii) Exchange $O$ and $\times$.

(iii) Construct a bow variety from the new bow diagram.

Higgs branch $\mathfrak{M}_{0}(\underline{\mathbf{v}}, \underline{\mathbf{w}})$ :

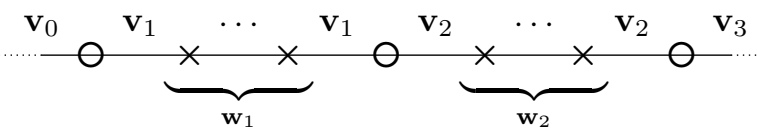

Coulomb branch $\mathcal{M}(\underline{\mathbf{v}}, \underline{\mathbf{w}})$ :

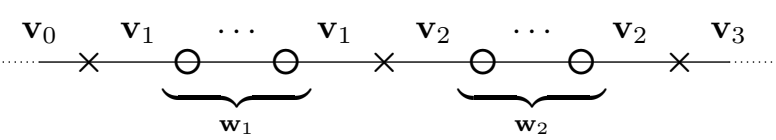

In fact, the Coulomb branch defined in $\S 6$ is the same one obtained by this procedure.

\subsection{Hanany-Witten transition as an isomorphism of bow varieties}

In a brane configuration, exchange of positions of a D5-brane and its next NS5-brane generates new D3-branes [HW97].

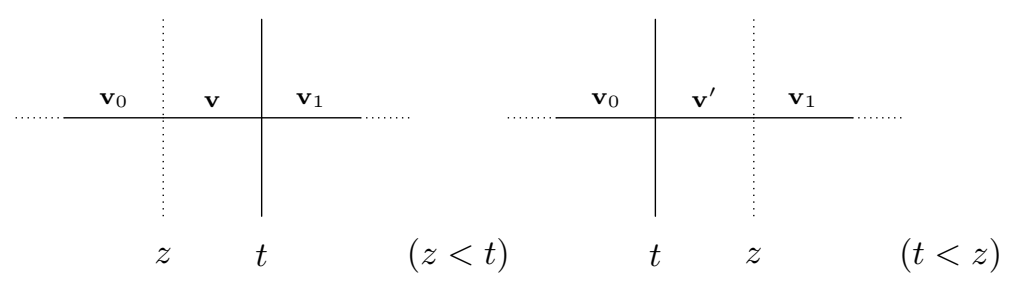

We interpret this transition in a language of bow variety.

Proposition 7.1 (Hanany-Witten transition). Set $m+m^{\prime}=l+n+1\left(m, m^{\prime}, l, n \geq 0\right)$. Then there exists a $\mathrm{GL}(l) \times \mathrm{GL}(n)$-equivariant isomorphism, preserving holomorphic symplectic structures;

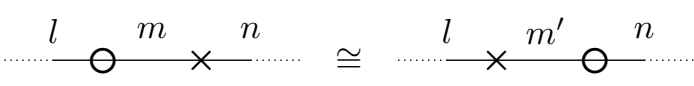

Here quotients are taken at only $m$ and $m^{\prime}$. Moreover both $\nu^{\mathbb{R}}$-stability and $\nu^{\mathbb{R}}$-semistability conditions are preserved under the isomorphism. 
By abuse of terminology, the corresponding transition of bow diagrams is also called HananyWitten transition. (Recall dimension vectors are parts of bow diagrams.)

Proof. Consider the following part of bow data:

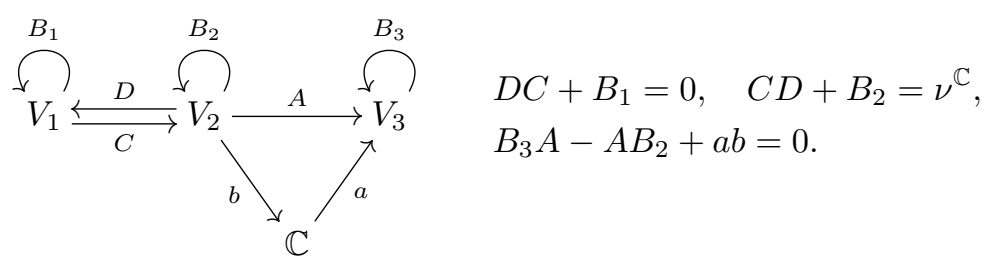

Like Proposition 2.4, we consider a three term complex

$$
V_{2} \stackrel{\alpha=\left[\begin{array}{c}
D \\
A \\
b
\end{array}\right]}{\longrightarrow} V_{1} \oplus V_{3} \oplus \mathbb{C} \stackrel{\beta=\left[A C\left(B_{3}-\nu^{\mathbb{C}}\right) a\right]}{\longrightarrow} V_{3},
$$

where $\beta \alpha=0$ and $\alpha$ is injective. Let

$$
V_{2}^{\text {new }}:=\operatorname{Coker} \alpha
$$

We define new bow data by

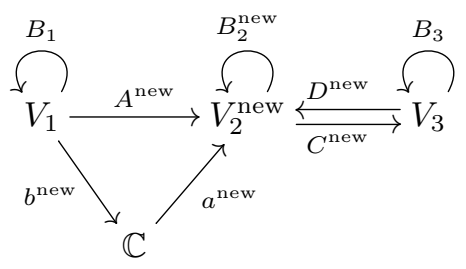

where $A^{\text {new }}, a^{\text {new }}$ are composition of inclusions of $V_{1}, \mathbb{C}$, to $V_{1} \oplus V_{3} \oplus \mathbb{C}$ and the projection $V_{1} \oplus V_{3} \oplus \mathbb{C} \rightarrow V_{2}^{\text {new }}$, and $D^{\text {new }}$ is the corresponding composition for $V_{3}$, but we change the sign. The remaining are as follows: $b^{\text {new }}=b C, C^{\text {new }}$ is a homomorphism induced from $\beta$, and $B_{2}^{\text {new }}=-D^{\text {new }} C^{\text {new }}$.

Let us check the defining equations. Recall $-D^{\text {new }}$ is the composition of $V_{3} \rightarrow V_{1} \oplus V_{3} \oplus \mathbb{C} \rightarrow$ $V_{2}^{\text {new }}$. Since $C^{\text {new }}$ is induced from $\beta,-C^{\text {new }} D^{\text {new }}$ is nothing but the composition of the inclusion $V_{3} \rightarrow V_{1} \oplus V_{3} \oplus \mathbb{C}$ and $\beta$, which is equal to $B_{3}-\nu^{\mathbb{C}}$.

Next consider

$$
V_{1} \stackrel{\alpha^{\text {new }}=\left[\begin{array}{c}
-B_{1} \\
C^{\text {new }} A^{\text {new }} \\
b^{\text {new }}
\end{array}\right]}{\longrightarrow} V_{1} \oplus V_{3} \oplus \mathbb{C} \stackrel{\beta^{\text {new }}=\left[A^{\text {new }}-D^{\text {new }} a^{\text {new }}\right]}{\longrightarrow} V_{2}^{\text {new }},
$$

where $\beta^{\text {new }}$ is nothing but the natural projection. We have

$$
\alpha^{\text {new }}=\left[\begin{array}{c}
-B_{1} \\
A C \\
b C
\end{array}\right]=\alpha C .
$$

Therefore $\beta^{\text {new }} \alpha^{\text {new }}=0$, which is nothing but the remaining defining equation.

Let us check the condition (S1). Take a subspace $S \subset V_{1}$ such that $B_{1}(S) \subset S, A^{\text {new }}(S)=0=$ $b^{\text {new }}(S)$. Observe that $A^{\text {new }}(S)=0$ means $S \oplus 0 \oplus 0 \subset \operatorname{Im} \alpha$. Let us consider $\tilde{S}=\alpha^{-1}(S \oplus 0 \oplus 0)$. Then $D(\tilde{S})=S$ and $A(\tilde{S})=0=b(\tilde{S})$. Therefore

$$
\alpha\left(\nu^{\mathbb{C}}-B_{2}\right)(\tilde{S})=\alpha C D(\tilde{S})=\alpha C(S)=\alpha^{\text {new }}(S)=\left[\begin{array}{c}
B_{1}(S) \\
0 \\
0
\end{array}\right] .
$$

The condition $B_{1}(S) \subset S$ implies $B_{2}(\tilde{S}) \subset \tilde{S}$. Hence $\tilde{S}=0$ thanks to (S1) for the original data. We have $S=0$ as well. 
Let us check the condition (S2). Suppose we have a subspace $T \subset V_{2}^{\text {new }}$ such that $B_{2}^{\text {new }}(T) \subset T$, $\operatorname{Im} A^{\text {new }}+\operatorname{Im} a^{\text {new }} \subset T$. We take its inverse image $\tilde{T}=\left(\beta^{\text {new }}\right)^{-1}(T)$ in $V_{1} \oplus V_{3} \oplus \mathbb{C}$. By the second assumption, it contains $V_{1} \oplus\{0\} \oplus \mathbb{C}$. Hence $\tilde{T}$ is a form of $V_{1} \oplus \bar{T} \oplus \mathbb{C}$ for $\bar{T} \subset V_{3}$. We also have $\operatorname{Im} \alpha \subset \tilde{T}$. Hence $A\left(V_{2}\right) \subset \bar{T}$. As $B_{2}^{\text {new }}=-D^{\text {new }} C^{\text {new }}$, the condition $B_{2}^{\text {new }}(T) \subset T$ implies $0 \oplus \beta(\tilde{T}) \oplus 0 \subset \tilde{T}$, i.e., $A C\left(V_{1}\right)+B_{3}(\bar{T})+a(\mathbb{C}) \subset \bar{T}$. Hence $\bar{T}=V_{3}$ thanks to (S2) for the original data. We have $T=V_{2}^{\text {new }}$ as well.

The inverse construction is clear. The original vector space $V_{2}$ is recovered from the new data as $\operatorname{Ker} \beta^{\text {new }}$. Note also $\beta^{\text {new }}$ is surjective thanks to (S2). Then $A, b, D$ are given as restrictions of projections $V_{1} \oplus V_{3} \oplus \mathbb{C}$ to $V_{3}, \mathbb{C}, V_{1}$ to $\operatorname{Ker} \beta^{\text {new }}$ (up to sign), $a$ is $C^{\text {new }} a^{\text {new }}$, and $C$ is $\alpha^{\text {new }}$ by (7.2). Finally we set $B_{2}=\nu^{\mathbb{C}}-C D$. The conditions (S1),(S2) for $\left(A, B_{2}, B_{3}, a, b\right)$ follow from the conditions $(\mathrm{S} 1),(\mathrm{S} 2)$ for $\left(A^{\text {new }}, B_{1}, B_{2}^{\text {new }}, a^{\text {new }}, b^{\text {new }}\right)$. We leave the details to the reader as an exercise.

Let us check the stability condition. Note that the isomorphism respects the group action by $\mathrm{GL}\left(V_{1}\right) \times \mathrm{GL}\left(V_{3}\right)$. As we can choose any segment $\zeta$ from a wavy line $\sigma$ in the numerical criterion, we could also choose any $\zeta$ in the definition of $\chi$ for the stability condition. In particular, if we choose $\operatorname{GL}\left(V_{1}\right)$ and $\operatorname{GL}\left(V_{3}\right)$ for $\chi$, it is clear that the stability condition is unchanged under the transition.

The proof of the compatibility of symplectic forms will be given in $\S \mathrm{A}$.

Remark 7.3. Let us sketch another proof of the last step checking $(\nu 1),(\nu 2)$ directly.

Let $S:=\bigoplus S_{i} \subset \bigoplus V_{i}$ be a graded subspace invariant under $A, B, C, D$ with $b(S)=0$ such that $\left.A\right|_{S_{2}}: S_{2} \rightarrow S_{3}$ is an isomorphism. We define $S_{2}^{\text {new }}:=A^{\text {new }}\left(S_{1}\right)$, i.e., the image of $S_{1} \oplus 0 \oplus 0$ under the projection $V_{1} \oplus V_{3} \oplus \mathbb{C} \stackrel{\beta^{\text {new }}}{\longrightarrow} V_{2}^{\text {new }}$. One can check that $S_{1} \oplus S_{2}^{\text {new }} \oplus S_{3}$ satisfies the conditions in $(\nu 1)$.

Conversely suppose a graded subspace $S^{\text {new }}:=S_{1} \oplus S_{2}^{\text {new }} \oplus S_{3} \subset V_{1} \oplus V_{2}^{\text {new }} \oplus V_{3}$ is given. We assume $S^{\text {new }}$ is invariant under $A^{\text {new }}, B^{\text {new }}, C^{\text {new }}, D^{\text {new }}$ with $b^{\text {new }}\left(S^{\text {new }}\right)=0$ and that $\left.A^{\text {new }}\right|_{S_{1}}: S_{1} \rightarrow S_{2}^{\text {new }}$ is an isomorphism. We define $S_{2} \subset V_{1} \oplus V_{3} \oplus \mathbb{C}$ as

$$
\left\{\left(\left.A^{\text {new }}\right|_{S_{1}}\right)^{-1} D^{\text {new }}(s) \oplus s \oplus 0 \mid s \in S_{3}\right\} .
$$

It is contained in Ker $\beta^{\text {new }}$, hence regarded as a subspace of $V_{2}$. One can check that $S_{1} \oplus S_{2} \oplus S_{3}$ satisfies the conditions in $(\nu 1)$.

Let $T:=\bigoplus T_{i} \subset \bigoplus V_{i}$ be a graded subspace invariant under $A, B, C, D$ with $\operatorname{Im} a \subset T$ such that $A: V_{2} / T_{2} \rightarrow V_{3} / T_{3}$ is an isomorphism. (Here the induced map is denoted by the same symbol A.) We define $\widetilde{T}_{2}^{\text {new }} \subset V_{1} \oplus V_{3} \oplus \mathbb{C}$ as

$$
\widetilde{T}_{2}^{\text {new }}:=\left\{v_{1} \oplus v_{3} \oplus z \mid v_{1} \bmod T_{1}=D A^{-1}\left(v_{3} \bmod T_{3}\right)\right\} .
$$

Then we define $T_{2}^{\text {new }}$ as the image of $\widetilde{T}_{2}^{\text {new }}$ under $\beta^{\text {new }}$. One can check that $T_{1} \oplus T_{2}^{\text {new }} \oplus T_{3}$ satisfies the conditions in $(\nu 2)$.

Let $T^{\text {new }}:=T_{1} \oplus T_{2}^{\text {new }} \oplus T_{3} \subset V_{1} \oplus V_{2}^{\text {new }} \oplus V_{3}$ be a graded subspace invariant under $A^{\text {new }}$, $B^{\text {new }}, C^{\text {new }}, D^{\text {new }}$ with $\operatorname{Im} a^{\text {new }} \subset T^{\text {new }}$ such that $A^{\text {new }}: V_{1} / T_{1} \rightarrow V_{2}^{\text {new }} / T_{2}^{\text {new }}$ is an isomorphism. We define $T_{2}:=A^{-1}\left(T_{3}\right)$. One can check that $T_{1} \oplus T_{2} \oplus T_{3}$ satisfies the conditions in $(\nu 2)$.

Since $S_{1}, S_{3}, T_{1}, T_{3}$ are unchanged, the original data satisfy $(\nu 1)$ (resp. $(\nu 2)$ ) if and only if the new data satisfy $(\nu 1)$ (resp. $(\nu 2))$.

\subsection{Invariants}

In this subsection we introduce invariants which are preserved under Hanany-Witten transition.

Recall two numbers defined in $§ 2.2$;

$$
N_{h}=\mathbf{v}_{\mathrm{i}(h)}-\mathbf{v}_{\mathrm{o}(h)}, \quad N_{x}=\mathbf{v}_{\zeta^{-}}-\mathbf{v}_{\zeta^{+}} .
$$

Lemma 7.4. Suppose $\zeta^{-}=[\mathrm{i}(h), x]$. Then $\left(N_{h}, N_{x}\right)=(m,-n)$ is transferred to $(m-1,-n-1)$ under Hanany-Witten transition \%.1. 
Proof. This is because that Hanany-Witten transition means

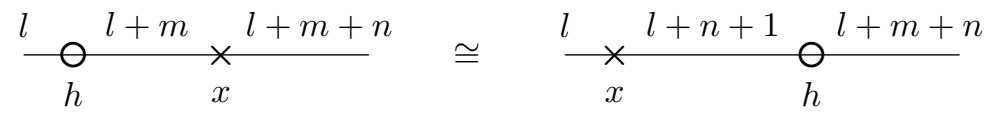

We define two numbers:

$$
\begin{aligned}
& N\left(h_{i}, h_{i+1}\right):=N_{h_{i}}-N_{h_{i+1}}+\left\{\text { the number of } \times \text { between } h_{i+1} \rightarrow h_{i}\right\} \\
& N\left(x_{i}, x_{i+1}\right):=N_{x_{i}}-N_{x_{i+1}}+\left\{\text { the number of } \bigcirc \text { between } x_{i} \rightarrow x_{i+1}\right\}
\end{aligned}
$$

where we number $h_{i}$ clockwise, while $x_{i}$ anti-clockwise. Here 'between $h_{i+1} \rightarrow h_{i}$ ' means that on the arc starting from $h_{i+1}$ towards $h_{i}$ in the anti-clockwise direction. For example, we have

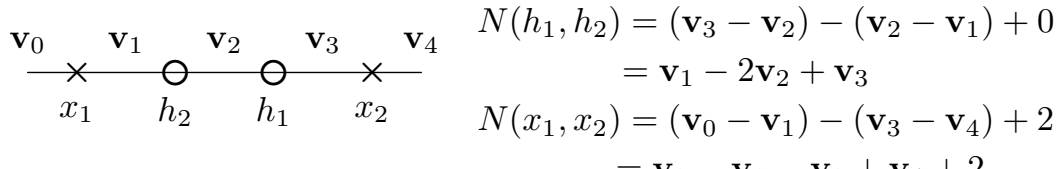

$$
\begin{aligned}
& =\mathbf{v}_{0}-\mathbf{v}_{1}-\mathbf{v}_{3}+\mathbf{v}_{4}+2 \text {. }
\end{aligned}
$$

We suppose the numbers $\ell, n$ of $\bigcirc$ and $\times$ are greater than 1 so that $h_{i} \neq h_{i+1}, x_{i} \neq x_{i+1}$. Here we understand $i, i+1$ modulo either $n$ or $\ell$.

Proposition 7.5. $N\left(h_{i}, h_{i+1}\right)$ and $N\left(x_{i}, x_{i+1}\right)$ are invariant under Hanany-Witten transition.

Proof. Recall that Lemma 7.4 states that $\left(N_{h_{i}}, N_{x_{j}}\right)=(m,-n)$ is transferred to $(m-1,-n-1)$.

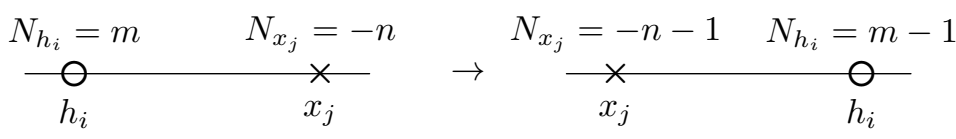

In the transition, the number of $\times$ between $h_{i}$ and $h_{i-1}$ decreases one and the number of $\times$ between $h_{i+1}$ and $h_{i}$ increases one. (This remains true even if $h_{i+1}=h_{i-1}$ since we are counting $\times$ on different intervals.) This implies $N\left(h_{i}, h_{i+1}\right)$ and $N\left(h_{i-1}, h_{i}\right)$ are invariant. This is the same for $N\left(x_{i}, x_{i+1}\right)$ and $N\left(x_{i-1}, x_{i}\right)$.

Lemma 7.6. $-N_{h}^{2}+\mathbf{v}_{\zeta^{+}}+\mathbf{v}_{\zeta^{-}}$and $-N_{x}^{2}+\mathbf{v}_{\mathbf{i}(h)}+\mathbf{v}_{\mathrm{o}(h)}$ are invariant under Hanany-Witten transition $\% .1$.

Proof. Let $l, m, n$ as in the proof of Lemma 7.4. Then $-N_{h}^{2}+\mathbf{v}_{\zeta^{+}}+\mathbf{v}_{\zeta^{-}}$is equal to

$$
-m^{2}+2(l+m)+n
$$

before the transition, and

$$
-(m-1)^{2}+2 l+n+1
$$

after the transition. They are equal. Since changes of dimensions remain the same even if we exchange $\bigcirc$ and $\times$, the same is true for $-N_{x}^{2}+\mathbf{v}_{\mathrm{i}(h)}+\mathbf{v}_{\mathrm{O}(h)}$.

The number $-N_{h}^{2}+\mathbf{v}_{\zeta^{+}}+\mathbf{v}_{\zeta^{-}}$is the contribution of these parts to the dimension of the bow variety. Since Hanany-Witten transition is an isomorphism of bow varieties, this result is natural. 


\subsection{Coulomb branches of type $A$ and nilpotent orbits}

Suppose that a framed quiver gauge theory of type $A_{n-1}$ with dimension vectors $\underline{\mathbf{v}}$, $\underline{\mathbf{w}}$ is given. We assign two $\mathfrak{s l}(n)$-weights $\lambda:=\sum_{i=1}^{n-1} \mathbf{w}_{i} \Lambda_{i}, \mu:=\sum_{i=1}^{n-1} \mathbf{w}_{i} \Lambda_{i}-\mathbf{v}_{i} \alpha_{i}$. Here $\Lambda_{i}$ is the $i$ th fundamental weight, and $\alpha_{i}$ is the $i$ th simple root. This is the same assignment as one appeared in quiver varieties. Let $\ell=\sum_{i} \mathbf{w}_{i}$ be the level of $\underline{\mathbf{w}}$.

We consider the corresponding bow diagram satisfying the balanced condition as in (6.1) so that the associated bow variety $\mathcal{M}(\underline{\mathbf{v}}, \underline{\mathbf{w}})$ is the Coulomb branch of the framed quiver gauge theory of finite type $A$. We number $\times$ and $\mathrm{O}$ as:

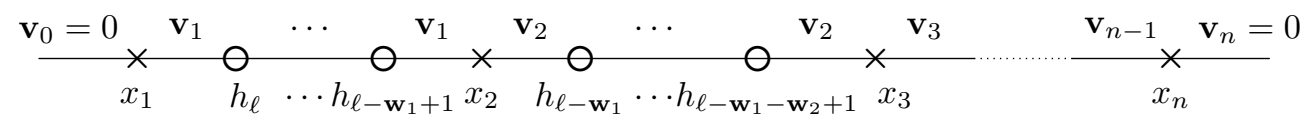

We set $\Lambda_{i}=[\underbrace{1, \ldots, 1}_{i}, 0, \ldots, 0], \alpha_{i}=\left[0, \ldots, 0,{\underset{i}{i+1}}_{i+1}-1, \ldots, 0\right] \in \mathbb{Z}^{n}$ as usual, and regard $\lambda, \mu$ as elements in $\mathbb{Z}^{n}$ (or $\mathfrak{g l}_{n}$-weights). Concretely

$$
\begin{gathered}
\lambda=\left[\lambda_{1}, \lambda_{2}, \cdots, \lambda_{n}\right], \quad \mu=\left[\mu_{1}, \mu_{2}, \cdots, \mu_{n}\right] \\
\lambda_{i}=\sum_{j \geq i} \mathbf{w}_{j}, \mu_{i}=\mathbf{v}_{n-1}+\sum_{j \geq i} \mathbf{u}_{j}, \quad \text { for } i=1,2, \cdots, n-1, \lambda_{n}=0, \mu_{n}=\mathbf{v}_{n-1},
\end{gathered}
$$

where $\underline{\mathbf{u}}=\underline{\mathbf{w}}-C \underline{\mathbf{v}}=\left(\mathbf{w}_{i}+\mathbf{v}_{i-1}+\mathbf{v}_{i+1}-2 \mathbf{v}_{i}\right)_{i=1}^{n-1}$. (We set $\mathbf{v}_{n}=0$.) Then $\underline{\mathbf{u}} \in \mathbb{Z}_{\geq 0}^{n-1}$ if and only if $\mu$ is dominant, i.e., $\mu_{1} \geq \mu_{2} \geq \cdots \geq \mu_{n}$. When $\mu$ is dominant, it is regarded as a partition with at most $n$ rows. On the other hand, $\lambda$ is always dominant, and is equal to ${ }^{t}\left(1^{w_{1}} 2^{w_{2}} \ldots(n-1)^{w_{n-1}}\right)$ where ${ }^{t}$ is the transposed partition. Note also $|\lambda|=|\mu|$, where || denote the sum of entries. (For a partition corresponding to a Young diagram, it is the total number of boxes.) We have $\lambda_{1}-\mu_{1}=\mathbf{v}_{1}, \lambda_{1}+\lambda_{2}-\left(\mu_{1}+\mu_{2}\right)=\mathbf{v}_{2}, \ldots,\left(\lambda_{1}+\cdots+\lambda_{n-1}\right)-\left(\mu_{1}+\cdots+\mu_{n-1}\right)=\mathbf{v}_{n-1}$. Thus $\lambda \geq \mu$ in the dominance order if and only if $\sum_{j=1}^{i} \lambda_{j} \geq \sum_{j=1}^{i} \mu_{j}$ for any $i$.

Let us write ${ }^{t} \lambda$ as $\left[{ }^{t} \lambda_{1},{ }^{t} \lambda_{2}, \ldots,{ }^{t} \lambda_{\ell}\right]$ with $\ell=\lambda_{1}=\sum_{i} \mathbf{w}_{i}$. For example, ${ }^{t} \lambda_{1}$ is the last $j$ with $\mathbf{w}_{j} \neq 0$, etc.

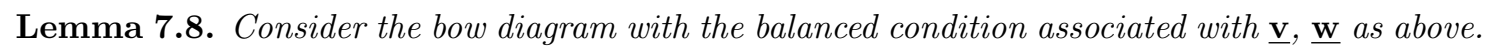

(1) $N\left(h_{i}, h_{i+1}\right)={ }^{t} \lambda_{i}-{ }^{t} \lambda_{i+1}$. In particular, $N\left(h_{i}, h_{i+1}\right)$ is always nonnegative.

(2) $N\left(x_{i}, x_{i+1}\right)$ is the ith entry of $\underline{\mathbf{u}}=\underline{\mathbf{w}}-C \underline{\mathbf{v}}$. In particular, $N\left(x_{i}, x_{i+1}\right) \geq 0$ for any $i$ if and only if $\mu=\sum \mathbf{w}_{i} \Lambda_{i}-\mathbf{v}_{i} \alpha_{i}$ is dominant.

Proof. (1) Since $N_{h_{i}}=0$ by the balanced condition, $N\left(h_{i}, h_{i+1}\right)$ is the number of $\times$ between $h_{i+1}$ and $h_{i}$. For example $N\left(h_{1}, h_{2}\right)=j_{1}-j_{2}$ if $h_{1}$ (resp. $h_{2}$ ) is between $x_{j_{1}}$ and $x_{j_{1}+1}$ (resp. $x_{j_{2}}$ and $\left.x_{j_{2}+1}\right)$. Now $j_{1}={ }^{t} \lambda_{1}, j_{2}={ }^{t} \lambda_{2}$ by definition. Hence $N\left(h_{1}, h_{2}\right)={ }^{t} \lambda_{1}-{ }^{t} \lambda_{2}$. General cases are the same.

(2) We calculate $N\left(x_{i}, x_{i+1}\right)$ as

$$
\begin{aligned}
N\left(x_{i}, x_{i+1}\right) & =\left(\mathbf{v}_{i-1}-\mathbf{v}_{i}\right)-\left(\mathbf{v}_{i}-\mathbf{v}_{i+1}\right)+\mathbf{w}_{i} \\
& =\mathbf{v}_{i-1}-2 \mathbf{v}_{i}+\mathbf{v}_{i+1}+\mathbf{w}_{i}=\mathbf{u}_{i} .
\end{aligned}
$$

Let us apply Hanany-Witten transition to move $\bigcirc$ to the left and $\times$ to the right. Then we get $(7.9)$

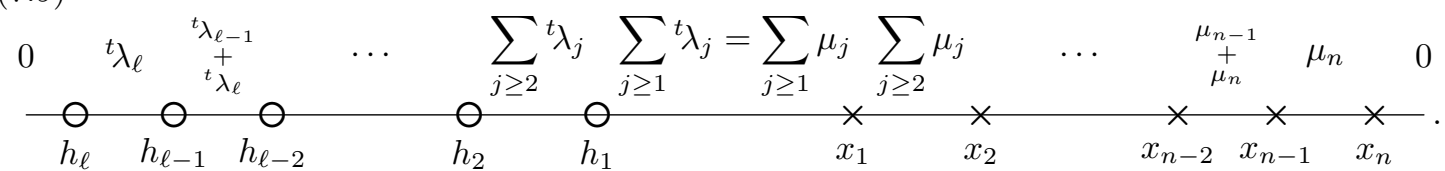

We have

$$
\begin{array}{cccc}
N_{h_{1}}={ }^{t} \lambda_{1}, & N_{h_{2}}={ }^{t} \lambda_{2}, & \ldots, & N_{h_{\ell}}={ }^{t} \lambda_{\ell}, \\
N_{x_{1}}=\mu_{1}, & N_{x_{2}}=\mu_{2}, & \ldots, & N_{x_{n}}=\mu_{n}
\end{array}
$$


for this bow diagram. Since $\lambda$ is dominant, we have $0 \leq N_{h_{1}} \leq N_{h_{2}} \leq \cdots \leq N_{h_{\ell}}$.

It is convenient for us to write $N_{h_{\sigma}}$ and $N_{x_{i}}$, rather than dimensions themselves, on $\bigcirc$ and $X$ in the bow diagram. If we write a dimension of a vector space on a particular segment (like 0 in the above diagram), other dimensions are calculated from $N_{h_{\sigma}}$ and $N_{x_{i}}$. We will use such a notation often hereafter.

For a finite type $A$, a quiver variety $\mathfrak{M}_{0}(\underline{\mathbf{v}}, \underline{\mathbf{w}})$ with dominant $\mu$ is isomorphic to an intersection of nilpotent orbit and transversal slice:

Theorem 7.10 ([Nak94b, Theorem 8.4]). Suppose $\mu$ is dominant. We have

$$
\mathfrak{M}_{0}(\underline{\mathbf{v}}, \underline{\mathbf{w}}) \cong \overline{\mathcal{N}}\left({ }^{t} \mu\right) \cap \mathcal{S}\left({ }^{t} \lambda\right),
$$

where $\mathcal{N}$ is a nilpotent orbit, $\overline{\mathcal{N}}$ is its closure, $\mathcal{S}$ is a transversal slice, and ${ }^{t} \mu$ is a partition which is represented by a transpose of Young diagram of a partition $\mu$.

And it is known what is the corresponding Coulomb branch by [BFN16b]:

Theorem 7.11. The Coulomb branch $\mathcal{M}(\underline{\mathbf{v}}, \underline{\mathbf{w}})$ of a quiver gauge theory with dominant $\mu$ is $\overline{\mathcal{N}}(\lambda) \cap \mathcal{S}(\mu)$, where $\lambda$ and $\mu$ are given by $(7.7)$.

Note that $\lambda, \mu$ are swapped and taken transpose for $\mathfrak{M}$ and $\mathcal{M}$.

Let us give proofs of both Theorem 7.10, and Theorem 7.11 by the procedure in $\S 7.1$.

A quiver variety $\mathfrak{M}_{0}(\underline{\mathbf{v}}, \underline{\mathbf{w}})$ corresponds to a bow diagram with the cobalanced condition Theorem 2.15. It is obtained from the bow diagram for $\mathcal{M}(\underline{\mathbf{v}}, \underline{\mathbf{w}})$ by exchanging $\times$ and $\mathrm{O}$ by $\$ 7.1$. Looking at (7.9), we find that it exchanges two partitions $\lambda$ and $\mu$ after taking their transposes. Thus it is enough to prove Theorem 7.11.

We divide (7.9) at the middle and consider $\mathcal{M}(\underline{\mathbf{v}}, \underline{\mathbf{w}})$ as the symplectic reduction of the product of varieties corresponding to the left and right sides by the action of GL $(r)$ with $r=\sum^{t} \lambda_{j}$. The left hand side, as a quiver variety of type $A$, has nonzero $W$ at the rightmost end. It is the variety originally appeared in [KP79]. Note that the dominance condition is automatically satisfied, and $\lambda$ is a partition. The variety is isomorphic to $\overline{\mathcal{N}}(\lambda)$. The right hand side, viewed as the moduli space of solutions of Nahm's equations on an interval, gives $\operatorname{GL}(r) \times \mathcal{S}(\mu)$ by [Bie97]. Thus, we obtain Theorem 7.11 .

Remark 7.12. We can also generalize these results to cases of deformation and resolutions. Let us state the results for only special cases for brevity.

Let $\nu=\left(\nu^{\mathbb{C}}, 0\right)$ be a parameter with vanishing real part. The left hand side of $(7.9)$ gives the closure $\overline{\mathcal{O}}$ of a conjugacy class $\mathcal{O}$ determined by $\nu^{\mathbb{C}}$ and $\lambda$. (See e.g., [CB03b].) The above argument show that $\mathcal{M}_{\nu}(\underline{\mathbf{v}}, \underline{\mathbf{w}}) \cong \overline{\mathcal{O}} \cap \mathcal{S}(\mu)$.

Let $\nu=\left(0, \nu^{\mathbb{R}}\right)$ be a parameter with vanishing complex part. We further assume $\nu_{\sigma}^{\mathbb{R}}>0$ for all $\sigma$. Then the left hand side of (7.9) gives the cotangent bundle $T^{*} \mathcal{F}$ of a partial flag variety $\mathcal{F}$ of type $A$. We have a projective morphism $\pi: T^{*} \mathcal{F} \rightarrow \overline{\mathcal{N}}(\lambda)$. (See [Nak94b, Th. 7.3].) Then $\mathcal{M}_{\nu}(\underline{\mathbf{v}}, \underline{\mathbf{w}}) \cong \pi^{-1}(\mathcal{S}(\mu))$.

We also have the corresponding results for quiver varieties. The second case $\nu=\left(0, \nu^{\mathbb{R}}\right)$ recovers [Maf05, Th. 8], which was conjectured in [Nak94b, Conj. 8.6].

\subsection{Stratification of Coulomb branches of type $A$}

We continue to work on the Coulomb branch of a framed quiver gauge theory of finite type $A_{n-1}$. If $\mu$ is dominant, we have $\mathcal{M}_{C} \cong \overline{\mathcal{N}}(\lambda) \cap \mathcal{S}(\mu)$, and we have a stratification $\mathcal{M}_{C}=\bigsqcup_{\kappa} \mathcal{N}(\kappa) \cap \mathcal{S}(\mu)$, where $\kappa$ runs dominant weights between $\mu$ and $\lambda$, i.e., $\mu \leq \kappa \leq \lambda$. We give a stratification in general in this subsection.

It is easier to state results in terms of $\mathfrak{s l}(n)$-weights $\lambda:=\sum_{i=1}^{n-1} \mathbf{w}_{i} \Lambda_{i}, \mu:=\lambda-\sum_{i=1}^{n-1} \mathbf{v}_{i} \alpha_{i}$. Let us write $\mathcal{M}(\mu, \lambda)$ instead of $\mathcal{M}(\underline{\mathbf{v}}, \underline{\mathbf{w}})$. 
Theorem 7.13. We have a stratification

$$
\mathcal{M}(\mu, \lambda)=\bigsqcup_{\mu \leq \kappa \leq \lambda} \mathcal{M}^{\mathrm{s}}(\mu, \kappa)
$$

where $\kappa$ runs dominant weights between $\mu$ and $\lambda$. We have $\mathcal{M}^{\mathrm{s}}(\mu, \kappa) \neq \emptyset$ for any $\kappa$. Moreover a transversal slice to $\mathcal{M}^{\mathrm{s}}(\mu, \kappa)$ in $\mathcal{M}(\mu, \lambda)$ is isomorphic to $\mathcal{M}(\kappa, \lambda)$.

These properties were originally proved in [Nak15] based on the description of Coulomb branches as moduli spaces of singular monopoles, which is not justified yet. Our argument gives a rigorous proof for type $A$. (See also [BFN16b, Rem. 3.19].)

Proof. Let us regard $\lambda, \mu \in \mathbb{Z}^{n}$ as in (7.7).

As in $§ 7.4$, we start with a bow variety satisfying the balanced condition and apply HananyWitten transition to move $O$ to the left and $\times$ to the right. We divide it at the middle and consider $\mathcal{M}(\mu, \lambda)$ as the symplectic reduction of the product of varieties from the left and right by $\operatorname{GL}(r)$ with $r=\sum \lambda_{i}$. The left side is $\overline{\mathcal{N}}(\lambda)$ as before. The right side is another bow variety $\widetilde{\mathcal{M}}(\mu)$ associated with

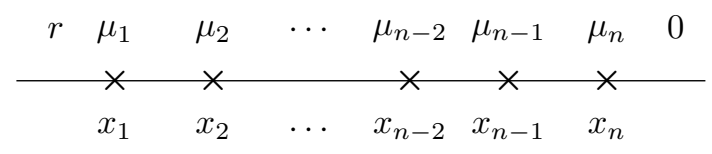

where we do not take the quotient by the leftmost GL $(r)$. Here we denote dimensions on the leftmost and rightmost segments, and $N_{x_{i}}$.

The stratification

$$
\overline{\mathcal{N}}(\lambda)=\bigsqcup_{\kappa \leq \lambda} \mathcal{N}(\kappa)
$$

by orbits induces a stratification of $\mathcal{M}(\mu, \lambda)$ as

$$
\mathcal{M}(\mu, \lambda)=\bigsqcup(\mathcal{N}(\kappa) \times \widetilde{\mathcal{M}}(\mu)) / / \mathrm{GL}(r)=\bigsqcup \Phi^{-1}(\mathcal{N}(\kappa)) / / \mathrm{GL}(r)
$$

where $\Phi: \widetilde{\mathcal{M}}(\mu) \rightarrow \mathfrak{g l}(r)$ is the moment map. Moreover $\Phi^{-1}(\overline{\mathcal{N}}(\kappa)) / / \mathrm{GL}(r)$ is a bow variety associated with the bow diagram (7.9) with $\lambda$ replaced by $\kappa$, that is

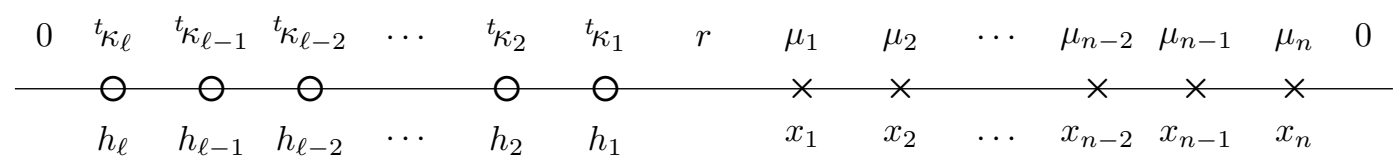

Let us also note that the stratification (7.14) is a special case of the stratification for quiver varieties (see e.g., [Nak94b, Prop. 6.7]):

$$
\mathfrak{M}_{0}\left(\underline{\mathbf{v}}^{\prime}, \underline{\mathbf{w}}^{\prime}\right)=\bigsqcup \mathfrak{M}_{0}^{\mathrm{reg}}\left(\underline{\mathbf{v}}_{0}^{\prime}, \underline{\mathbf{w}}^{\prime}\right)
$$

where $\underline{\mathbf{w}}^{\prime}=(r, 0, \ldots, 0), \underline{\mathbf{v}}^{\prime}=\left(\sum_{j \geq i}{ }^{t} \lambda_{j}\right)_{i=1}^{\ell}, \underline{\mathbf{v}}_{0}^{\prime}=\left(\sum_{j \geq i}{ }^{t} \kappa_{j}\right)_{i=1}^{\ell}$. The dominance condition ${ }^{t} \kappa_{1} \geq{ }^{t} \kappa_{2} \geq \cdots \geq{ }^{t} \kappa_{\ell}$ is a special case of the necessary condition for $\mathfrak{M}_{0}^{\mathrm{reg}}\left(\underline{\mathbf{v}}_{0}^{\prime}, \underline{\mathbf{w}}^{\prime}\right) \neq \emptyset$ in [Nak94b, Lem. 8.1], [Nak98, Lem. 4.7].

Claim. We have $n \geq{ }^{t} \kappa_{1}$ if $\Phi^{-1}(\mathcal{N}(\kappa)) \neq \emptyset$.

We add a new $h_{0}$ at the right of $x_{n}$. Then we have $N_{h_{0}}=0$, and $N\left(h_{0}, h_{1}\right)=n-{ }^{t} \kappa_{1}$. We move $h_{0}$ to the right of $h_{1}$ by Hanany-Witten transitions. Since $N\left(h_{0}, h_{1}\right)$ is preserved, we have $N_{h_{0}}=n$ in the resulted bow diagram. Now we consider the left half of the resulted bow diagram 
as a quiver variety. We derive $n \geq{ }^{t} \kappa_{1}$ as a necessary condition for $\mathfrak{M}_{0}^{\text {reg }} \neq \emptyset$ as above. The claim is proved.

Let us apply Hanany-Witten transition to move $\times$ to the left so that $N_{h_{\sigma}}=0$ for $1 \leq \sigma \leq \ell$. Namely we move ${ }^{t} \kappa_{\ell}$ of $\times$ 's to the left of $h_{\ell}$, move $\left({ }^{t} \kappa_{\ell-1}-{ }^{t} \kappa_{\ell}\right)$ of $\times$ 's between $h_{\ell}$ and $h_{\ell-1}$, and so on. Since $N\left(h_{\sigma}, h_{\sigma+1}\right)$ does not change, it means all $N_{h_{\sigma}}$ are equal, but $N_{h_{\ell}}=0$ by the first move. This procedure is possible as $n \geq{ }^{t} \kappa_{1}$.

If $n={ }^{t} \kappa_{1}$, the last $x_{n}$ is moved between $h_{1}$ and $h_{2}$. But the the balanced condition is satisfied, and the dimension on the rightmost segment is 0 . Therefore dimensions on two segments, left and right of $h_{1}$ are both 0 . Thus we can remove $h_{1}$, and the resulted bow variety is associated with a framed quiver gauge theory of type $A_{n-1}$. The corresponding dimension vectors $\mathbf{v}^{\prime}, \mathbf{w}^{\prime}$ are determined by

$$
\kappa-\kappa_{n}[1,1, \ldots, 1]=\sum_{i} \mathbf{w}_{i}^{\prime} \Lambda_{i}, \quad \mu-\kappa_{n}[1,1, \ldots, 1]=\sum_{i} \mathbf{w}_{i}^{\prime} \Lambda_{i}-\mathbf{v}_{i}^{\prime} \alpha_{i} .
$$

Since we must have $\mathbf{v}_{i}^{\prime} \geq 0$, the strata are labeled by $\kappa$ with $\mu \leq \kappa \leq \lambda$. More precisely our $\kappa \in \mathbb{Z}^{n}$ is a $\mathfrak{g l}(n)$-weight, so must be changed to an $\mathfrak{s l}(n)$-weight by $(7.15)$.

We claim that the following condition is satisfied:

$$
\text { (H-S1): } B_{i}\left(S_{i}\right) \subset S_{i}, S_{i} \subset \operatorname{Ker} b_{i}, A_{i}\left(S_{i}\right) \subset S_{i+1} \Rightarrow S_{i}=0 .
$$

In fact, by Proposition 2.2, we have $\operatorname{dim} S_{1} \leq \operatorname{dim} S_{2} \leq \ldots$ Hence $S_{i}=0$ as $\operatorname{dim} S_{n+1}=0$. Now this condition implies that the $\operatorname{GL}(r)$-action on $\widetilde{\mathcal{M}}(\mu)$ is free. Therefore $\Phi^{-1}(\mathcal{N}(\kappa)) / / \operatorname{GL}(r)$ is smooth and symplectic.

Alternatively we can check the smoothness as follows. The stability conditions $(\nu 1),(\nu 2)($ with $\nu=0$ ) are equivalent to the condition $\mathfrak{M}_{0}^{\text {reg }}$ on the left side, as $S_{\zeta^{-}} \cong S_{\zeta^{+}}, V_{\zeta^{-}} / T_{\zeta^{-}} \cong V_{\zeta^{+}} / T_{\zeta^{+}}$ mean that $S_{\zeta}=0, T_{\zeta}=V_{\zeta}$ on segments right of $h_{1}$. Therefore $\Phi^{-1}(\mathcal{N}(\kappa)) / / \mathrm{GL}(r)$ coincides with $\mathcal{M}^{\mathrm{s}}(\mu, \kappa)$, hence is smooth. It also implies that this stratification coincides with (4.3), where we do not have the factor $S^{k}\left(\mathfrak{M}^{\mathrm{s}}(\delta)\right)$ as we are considering finite type $A$ bow varieties.

Let us prove $\lambda \geq \kappa$ from (4.3). Recall that the difference of dimension vectors $\underline{\mathbf{v}}-\underline{\mathbf{v}}^{\prime}$ in (4.3) has nonnegative entries. Thus $\sum_{j>i}{ }^{t} \lambda_{j} \geq \sum_{j>i}{ }^{t} \kappa_{j}$. This is equivalent to ${ }^{t} \lambda \leq{ }^{t} \kappa \Leftrightarrow \lambda \geq \kappa$.

Let us remark we also see that $\mathcal{M}^{\mathrm{s}}(\mu, \lambda) \neq \emptyset$ for $\mu \leq \lambda$ with dominant $\lambda$. In fact, we have $\mathcal{M}(\mu, \lambda) \neq \emptyset$ as it is a Coulomb branch. We have just observed $\mathcal{M}(\mu, \kappa)$ is a Coulomb branch, hence its dimension is $2 \sum \mathbf{v}_{i}^{\prime}$. This is strictly smaller than $2 \sum \mathbf{v}_{i}$ if $\kappa \neq \lambda$ since $\lambda>\kappa$. Therefore $\mathcal{M}^{\mathrm{s}}(\mu, \lambda) \neq \emptyset$.

Finally let us consider a local structure around a point $p$ in $\mathcal{M}^{\mathrm{s}}(\mu, \kappa)$. By Proposition 4.4 a transversal slice is given a quiver variety, which is of type $A$. It is clear that the slice is locally isomorphic to $\overline{\mathcal{N}}(\lambda) \cap \mathcal{S}(\kappa)$, which is $\mathcal{M}(\kappa, \lambda)$ by Theorem 7.11.

\subsection{Coulomb branches and quiver varieties of affine type $A$}

We now turn to affine type $A$. We take a framed quiver gauge theory of type affine $A_{n-1}$ with dimension vectors $\underline{\mathbf{v}}, \underline{\mathbf{w}}$. Let $\ell=\sum_{i} \mathbf{w}_{i}$ be the level of $\underline{\mathbf{w}}$.

We number $x$ and $O$ as

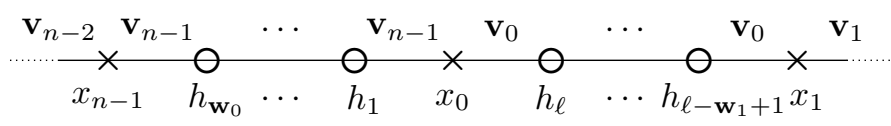

Note that $x_{0}, x_{1}, \ldots$ are in the anticlockwise order, while $h_{1}, h_{2}, \ldots$ are in the clockwise order.

Before discussing Coulomb branches, we review generalized Young diagrams and their relations to weights of affine Lie algebras.

Let $\mathfrak{s l}(n)_{\text {aff }}($ resp. $\widehat{\mathfrak{s l}}(n))$ be the affine Lie algebra of type $A_{n-1}$, which includes (resp. does not include) the degree operator $d$ in the Cartan subalgebra. We assign two $\mathfrak{s l}(n)_{\text {aff }}$ weights $\lambda:=\sum_{i=0}^{n-1} \mathbf{w}_{i} \Lambda_{i}+\mathbf{v}_{0} \delta, \mu:=\sum_{i=0}^{n-1} \mathbf{w}_{i} \Lambda_{i}-\mathbf{v}_{i} \alpha_{i}+\mathbf{v}_{0} \delta$ as in finite case. Our convention is $\left\langle d, \Lambda_{i}\right\rangle=0,\left\langle d, \alpha_{i}\right\rangle=\delta_{i 0}, \delta=\alpha_{0}+\cdots+\alpha_{n-1}$. We add $\mathbf{v}_{0} \delta$ so that $\langle d, \mu\rangle=0$. This is a different 
convention from the quiver variety setting, but simultaneous shifts of $\lambda, \mu$ by an element in $\mathbb{Z} \delta$ does not affect anything in practice. The projection $(\mathfrak{s l}(n)$ aff-weight lattice $) \rightarrow(\widehat{\mathfrak{s l}}(n)$-weight lattice $)$ is given by the quotient modulo $\mathbb{Z} \delta$.

We use the notion of a generalized Young diagram with the level $\ell$ constraint as in [Nak09, App. A]. A sequence of integers $\left[\lambda_{1}, \ldots, \lambda_{n}\right]$ is a generalized Young diagram with the level $\ell$ constraint if it satisfies the inequalities

$$
\begin{gathered}
\lambda_{1} \geq \lambda_{2} \geq \cdots \geq \lambda_{n}, \\
\lambda_{1}-\lambda_{n} \leq \ell .
\end{gathered}
$$

As in (7.7) we introduce two integral vectors $\left[\lambda_{1}, \lambda_{2}, \ldots, \lambda_{n}\right],\left[\mu_{1}, \mu_{2}, \ldots, \mu_{n}\right] \in \mathbb{Z}^{n}$ by

$$
\lambda_{i}=\sum_{j=i}^{n-1} \mathbf{w}_{j}, \quad \mu_{i}=\mathbf{v}_{n-1}-\mathbf{v}_{0}+\sum_{j=i}^{n-1} \mathbf{u}_{j}, \quad \text { for } i=1,2, \cdots, n-1, \lambda_{n}=0, \mu_{n}=\mathbf{v}_{n-1}-\mathbf{v}_{0}
$$

where $\underline{\mathbf{u}}=\underline{\mathbf{w}}-C \underline{\mathbf{v}}=\left(\mathbf{w}_{i}+\mathbf{v}_{i-1}+\mathbf{v}_{i+1}-2 \mathbf{v}_{i}\right)_{i=0}^{n-1}$. (The index is modulo $n$.) Then $\left[\lambda_{1}, \ldots, \lambda_{n}\right]$ is a generalized Young diagram with the level $\ell$ constraint. On the other hand,

$$
\begin{gathered}
\mu_{1}-\mu_{2}=\mathbf{u}_{1}, \quad \mu_{2}-\mu_{3}=\mathbf{u}_{2}, \quad \ldots, \quad \mu_{n-1}-\mu_{n}=\mathbf{u}_{n-1}, \\
\mu_{1}-\mu_{n}=\sum_{j=1}^{n-1} \mathbf{u}_{j}=\ell-\mathbf{u}_{0} .
\end{gathered}
$$

Therefore $\mu$ is dominant if and only if $\left[\mu_{1}, \ldots, \mu_{n}\right]$ is a generalized Young diagram with the level $\ell$ constraint. Note also

$$
|\lambda|:=\sum_{i=1}^{n} \lambda_{i}=\sum_{i=1}^{n} \mu_{i}=:|\mu|
$$

from the definition.

Remark that $\mu$ is not recovered from $\left[\mu_{1}, \ldots, \mu_{n}\right]$, as there is an ambiguity of $\mathbb{Z} \delta$. In fact, the set of generalized Young diagrams with the level $\ell$ constraint is bijective to the set of dominant $\widehat{\mathfrak{g l}}(n)$ weights of level $\ell$. The projection $\widehat{\mathfrak{g l}}(n)$-weights $\rightarrow \widehat{\mathfrak{s l}}(n)$-weights is identified with the quotient modulo shifts $\left[\mu_{1}, \ldots, \mu_{n}\right] \mapsto\left[\mu_{1}+k, \ldots, \mu_{n}+k\right]$ for $k \in \mathbb{Z}$. The above $|\lambda|$ is the charge, which is the eigenvalue of $J(0)$, the 0th Fourier mode of the Heisenberg algebra added to $\widehat{\mathfrak{s l}}(n)$ to get $\widehat{\mathfrak{g l}}(n)$. Hence the constraint $|\lambda|=|\mu|$ is natural. A generalized Young diagram together with an integer $\langle d, \lambda\rangle$ bijectively corresponds to a dominant $\mathfrak{g l}_{\text {aff }}(n)$-weights.

We have $\lambda_{1}-\mu_{1}+\mathbf{v}_{0}=\mathbf{v}_{1}, \lambda_{1}+\lambda_{2}-\left(\mu_{1}+\mu_{2}\right)+\mathbf{v}_{0}=\mathbf{v}_{2}, \ldots,\left(\lambda_{1}+\cdots+\lambda_{n-1}\right)-\left(\mu_{1}+\cdots+\mu_{n-1}\right)+$ $\mathbf{v}_{0}=\mathbf{v}_{n-1}$. Thus $\lambda \geq \mu$ in the dominance order if and only if $\sum_{j=1}^{i} \lambda_{j}+\langle d, \lambda-\mu\rangle \geq \sum_{j=1}^{i} \mu_{j}$ for any $i=1, \ldots, n$ and $\langle d, \lambda-\mu\rangle \geq 0$.

We draw a generalized Young diagram as

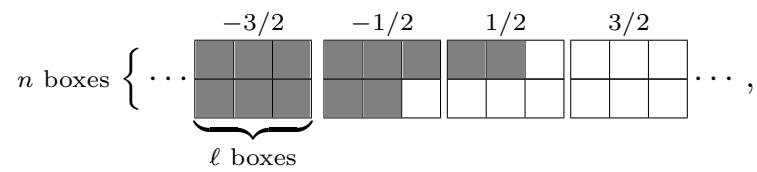

where a box is indexed by $(i, \sigma, N)$ with $1 \leq i \leq n, 1 \leq \sigma \leq \ell, N \in \mathbb{Z}+1 / 2$ and we put a gray box $\square$ if $\ell(N-1 / 2)+\sigma \leq \lambda_{i}$. The above figure is $\left[\lambda_{1}, \lambda_{2}\right]=[2,-1]$ for $n=2, \ell=3$.

We define the transpose of $\lambda$ by flipping each $n \times \ell$ rectangle along its diagonal. It has $\ell$ entries and satisfies the level $n$ constraint. The transpose describes the correspondence of dominant weights under the level-rank duality (see [Nak09, App. A]). Let us denote the transpose as $\left[{ }^{t} \lambda_{1},{ }^{t} \lambda_{2}, \ldots,{ }^{t} \lambda_{\ell}\right]$. The above example gives $\left[{ }^{t} \lambda_{1},{ }^{t} \lambda_{2},{ }^{t} \lambda_{3}\right]=[1,1,-1]$. Note that the transpose of a shifted diagram involves a 'vertical shift' of the transposed diagram. The vertical shift corresponds to a cyclic rotation of the affine Dynkin diagram of type $A_{n-1}$. Thus the transpose should be understood as a map from the set of level $\ell$ dominant $\widehat{\mathfrak{s l}}(n)$ weights to the set of level $n$ dominant $\widehat{\mathfrak{s l}}(\ell)$ weights modulo cyclic rotations.

Let us return back to the bow diagram with the balanced condition. 
Lemma 7.18. Consider the bow diagram with the balanced condition associated with dimension vectors $\underline{\mathbf{v}}, \underline{\mathbf{w}} \in \mathbb{Z}_{\geq 0}^{n}$ as in (6.1). Then the followings hold:

(1) $N\left(h_{\sigma}, h_{\sigma+1}\right)={ }^{t} \lambda_{\sigma}-{ }^{t} \lambda_{\sigma+1}$ for $1 \leq \sigma \leq \ell-1$. And $N\left(h_{\ell}, h_{1}\right)=n-\left({ }^{t} \lambda_{1}-{ }^{t} \lambda_{\ell}\right)$. In particular, $N\left(h_{\sigma}, h_{\sigma+1}\right)(1 \leq \sigma \leq \ell-1), N\left(h_{\ell}, h_{1}\right)$ are always nonnegative.

(2) $N\left(x_{i}, x_{i+1}\right)$ is equal to the ith entry of $\underline{\mathbf{u}}=\underline{\mathbf{w}}-C \underline{\mathbf{v}}$. Therefore $N\left(x_{i}, x_{i+1}\right) \geq 0$ for any $i$ if and only if $\sum \mathbf{w}_{i} \Lambda_{i}-\mathbf{v}_{i} \alpha_{i}$ is dominant.

Proof. The proof of (2) is exactly the same as in Lemma 7.8. The proof of (1) is also almost the same, as the nontrivial part of our diagram sits in the rectangle at $N=1 / 2$, and regarded as a usual Young diagram. Let us check the formula for $N\left(h_{\ell}, h_{1}\right)$. First note that $h_{\ell}\left(\right.$ resp. $\left.h_{1}\right)$ is between $x_{i}$ and $x_{i+1}$ (resp. $x_{j}$ and $x_{j+1}$ ) with $i={ }^{t} \lambda_{\ell}, j={ }^{t} \lambda_{1}$. Since we count the number of $\times$ in the $\operatorname{arc} h_{1} \rightarrow h_{\ell}$, it is equal to $n-(j-i)$.

Proposition 7.19. A bow diagram satisfying the balanced condition is determined by $N\left(x_{i}, x_{i+1}\right)$ $(0 \leq i \leq n-1), N\left(h_{\sigma}, h_{\sigma+1}\right)(1 \leq \sigma \leq \ell)$ plus either of

$$
-\sum_{\sigma=1}^{\ell} N_{h_{\sigma}}^{2}+\sum_{i=0}^{n-1}\left(\mathbf{v}_{\zeta_{i}^{-}}+\mathbf{v}_{\zeta_{i}^{+}}\right) \quad \text { or } \quad-\sum_{i=0}^{n-1} N_{x_{i}}^{2}+\sum_{\sigma=1}^{\ell}\left(\mathbf{v}_{\mathrm{i}\left(h_{\sigma}\right)}+\mathbf{v}_{\mathrm{o}\left(h_{\sigma}\right)}\right) .
$$

Here $\zeta_{i}^{-} \rightarrow x_{i} \rightarrow \zeta_{i}^{+}$. In particular, there is at most one bow diagram satisfying the balanced condition among those obtained by successive application of Hanany-Witten transitions.

Proof. Since $N\left(x_{i}, x_{i+1}\right), N\left(h_{\sigma}, h_{\sigma+1}\right)$ and two invariants above are preserved under HananyWitten transitions, the last assertion is a consequence of the first assertion.

Let us prove the first assertion. Consider a balanced bow diagram for $\underline{\mathbf{w}}$, ‥ Since $N\left(h_{\sigma}, h_{\sigma+1}\right)$ is the number of $\times$ between $h_{\sigma+1}$ and $h_{\sigma}$, the collection $\left\{N\left(h_{\sigma}, h_{\sigma+1}\right)\right\}_{\sigma=1}^{\ell}$ determines distribution of $\bigcirc$ and $x$, and hence $\underline{\mathbf{w}}$.

By Lemma $7.18(2),\left\{N\left(x_{i}, x_{i+1}\right)\right\}$ determines $\underline{\mathbf{w}}-C \underline{\mathbf{v}}$. It defines $\underline{\mathbf{v}}$ up to an addition of a multiple of ${ }^{t}(1,1, \ldots, 1)$.

If we add ${ }^{t}(1,1, \ldots, 1)$ to $\underline{\mathbf{v}}$, the above two invariants increase by $2 n$ and $2 \ell$ respectively. Therefore either of two invariants fixes the ambiguity.

Proposition 7.20. If $N\left(x_{i}, x_{i+1}\right) \geq 0$ for any $i$, the bow diagram is transformed to one with a cobalanced dimension vector by successive application of Hanany-Witten transitions of Proposition 7.1. (It is unique by Proposition 7.19.) Here we assume that the bow diagram has at least one $\mathrm{O}$.

Proof. We prove that all $N_{x_{i}}$ can be made simultaneously to 0 by applying Hanany-Witten transitions several times.

We first apply Hanany-Witten transition so that $\bigcirc$ appear only between $x_{0}$ and $x_{1}$ :

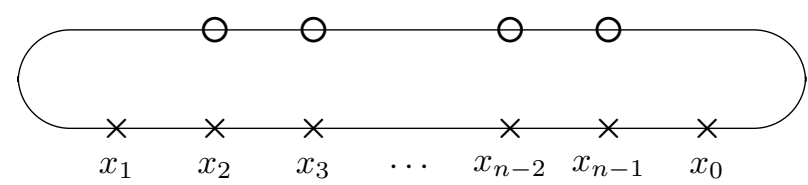

Then $N\left(x_{1}, x_{2}\right), N\left(x_{2}, x_{3}\right), \ldots, N\left(x_{n-2}, x_{n-1}\right), N\left(x_{n-1}, x_{0}\right), N\left(x_{0}, x_{1}\right)$ are all nonnegative and the sum is equal to $\ell$.

By Hanany-Witten transition, we move $N\left(x_{1}, x_{2}\right)$ of O's between $x_{1}$ and $x_{2}, N\left(x_{2}, x_{3}\right)$ of O's between $x_{2}$ and $x_{3}$, and so on until $N\left(x_{n-1}, x_{0}\right)$ of $O$ 's between $x_{n-1}$ and $x_{0}$. Then $N\left(x_{0}, x_{1}\right)$ of O's are remained between $x_{0}$ and $x_{1}$. Since $N\left(x_{i}, x_{i+1}\right)$ is unchanged, the resulted bow diagram has $N_{x_{0}}=N_{x_{1}}=\cdots=N_{x_{n-1}}=N_{x_{0}}$. 
And then we use Hanany-Witten transition $N_{x_{0}}$ times for all $\times$ simultaneously. Therefore, we can get a bow variety with $N_{x_{i}}=0$ for all $i$.

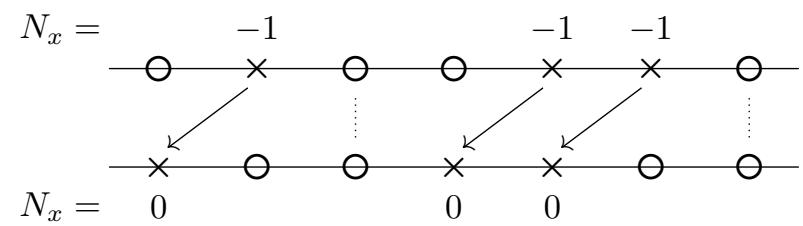

This final step is possible as the bow diagram has at least one $\bigcirc$.

Corollary 7.21. Consider a framed quiver gauge theory of affine type $A_{n-1}$ with dimension vectors $\underline{\mathbf{v}}, \underline{\mathbf{w}}$. Let $\ell$ be the level of $\underline{\mathbf{w}}$, which is assumed to be positive. Assume that $\underline{\mathbf{u}}=\underline{\mathbf{w}}-C \underline{\mathbf{v}} \in$ $\mathbb{Z}_{\geq 0}^{n}$. Then the Coulomb branch $\overline{\mathcal{M}}(\underline{\mathbf{v}}, \underline{\mathbf{w}})$ is isomorphic to a quiver variety $\mathfrak{M}_{0}\left(\underline{\mathbf{v}}^{\prime}, \underline{\mathbf{w}}^{\prime}\right)$ of affine type $A_{\ell-1}$ of level $n$.

Moreover $\underline{\mathbf{v}}^{\prime}, \underline{\mathbf{w}}^{\prime}$ are determined by two conditions: (a) Two generalized Young diagrams are swapped and taken transpose. (b) It satisfies

$$
2 \sum_{i=0}^{n-1} \mathbf{v}_{i}=\sum_{\sigma=0}^{\ell-1} 2 \mathbf{v}_{\sigma}^{\prime} \mathbf{w}_{\sigma}^{\prime}-\left(\mathbf{v}_{\sigma}^{\prime}-\mathbf{v}_{\sigma-1}^{\prime}\right)^{2}
$$

Note that $N\left(h_{\sigma}, h_{\sigma+1}\right) \geq 0$ for any $\sigma$, as it comes from a framed quiver gauge theory. Hence we have $\underline{\mathbf{w}}^{\prime}-C \underline{\mathbf{v}}^{\prime} \in \mathbb{Z}_{\geq 0}^{\ell}$, i.e., the weight $\sum_{\sigma} \mathbf{w}_{\sigma}^{\prime} \Lambda_{\sigma}^{\prime}-\mathbf{v}_{\sigma}^{\prime} \alpha_{\sigma}^{\prime}$ is dominant. Here $\Lambda_{\sigma}^{\prime}, \alpha_{\sigma}^{\prime}$ are fundamental and simple roots for $\mathfrak{s l}(\ell)$ aff .

Proof. If $n=1$, the cobalanced condition is automatically satisfied for a bow diagram arising from a framed quiver gauge theory.

If $n>1$, we get $N\left(x_{i}, x_{i+1}\right) \geq 0$ for any $i$ by Lemma 7.18. We now use Proposition 7.20 to get a bow variety with a cobalanced dimension vector. It is a quiver variety by Theorem 2.15.

Let us determine $\underline{\mathbf{v}}^{\prime}, \underline{\mathbf{w}}^{\prime}$ explicitly. Let us start from the bow diagram in (7.16) and move all $\bigcirc$ between $x_{0}$ and $x_{1}$ as above. We suppose that no $\bigcirc$ cross $x_{0}$ so that dimensions $\mathbf{v}_{0}, \mathbf{v}_{n-1}$ adjacent to $x_{0}$ are unchanged. We have $N_{x_{0}}=\mathbf{v}_{n-1}-\mathbf{v}_{0}=\mu_{n}$. Then $N_{x_{i}}=\mu_{i}$ follows by Lemma 7.18.

Since $h_{\ell}$ is between $x_{t_{\ell}}$ and $x_{t_{\ell}+1}$ in the original diagram, it crosses ${ }^{t} \lambda_{\ell}$ of $\times$ during the transitions. Therefore the dimension of the segment to the left of $h_{\ell}$ is ${ }^{t} \lambda_{\ell}+\mathbf{v}_{0}$. Hence $N_{h_{\ell}}={ }^{t} \lambda_{\ell}$. It follows that $N_{h_{\sigma}}={ }^{t} \lambda_{p}$ by Lemma 7.18. Thus we obtain

$$
\mathbf{v}_{0}+\sum_{i} i \mathbf{w}_{i}\left(\begin{array}{ccccccc} 
& { }^{t} \lambda_{1} & { }^{t} \lambda_{2} & & { }^{t} \lambda_{\ell-1} & { }^{t} \lambda_{\ell} & \\
& \wp & 0 & & \bigcirc & \bigcirc & \\
& h_{1} & h_{2} & \ldots & h_{\ell-1} & h_{\ell} & \\
& & & & & & \\
\mu_{1} & \mu_{2} & \mu_{3} & & \mu_{n-2} & \mu_{n-1} & \mu_{n} \\
\times & \times & \times & & \times & \times & \times \\
x_{1} & x_{2} & x_{3} & \cdots & x_{n-2} & x_{n-1} & x_{0}
\end{array} \mathbf{v}_{0}\right.
$$

Here numbers ${ }^{t} \lambda_{\sigma}, \mu_{i}$ above $\mathrm{O}, \times$ indicate the values of $N_{h_{\sigma}}, N_{x_{i}}$ respectively. Two numbers $\mathbf{v}_{0}$ and $\mathbf{v}_{0}+\sum_{i} i \mathbf{w}_{i}$ are dimensions of vector spaces on two segments, between $x_{0}$ and $h_{\ell}, h_{1}$ and $x_{1}$ respectively.

Note that the dimension on the segment between $x_{1}$ and $h_{1}$ are calculated from $\mathbf{v}_{0}$ by adding either $N_{x_{i}}$ or $N_{h_{\sigma}}$. The answer is $\mathbf{v}_{0}+\sum_{i} i \mathbf{w}_{i}$, as $|\lambda|=|\mu|=\sum_{i} i \mathbf{w}_{i}$.

We take a bow diagram with the cobalanced condition associated with dimension vectors $\left(\underline{\mathbf{v}}^{\prime}, \underline{\mathbf{w}}^{\prime}\right)$. The result should be the same as above. Then it is clear that the pair of two generalized Young diagrams associated with $\underline{\mathbf{w}}^{\prime}, \underline{\mathbf{v}}^{\prime}$ is $\left({ }^{t} \mu,{ }^{t} \lambda\right)$. The remaining constraint is

$$
2 \sum_{i=0}^{n-1} \mathbf{v}_{i}=-\sum_{p} N_{h_{\sigma}}^{2}+\sum_{i=0}^{n-1}\left(\mathbf{v}_{\zeta_{i}^{-}}+\mathbf{v}_{\zeta_{i}^{+}}\right)=\sum_{\sigma=0}^{\ell-1} 2 \mathbf{v}_{\sigma}^{\prime} \mathbf{w}_{\sigma}^{\prime}-\left(\mathbf{v}_{\sigma}^{\prime}-\mathbf{v}_{\sigma-1}^{\prime}\right)^{2}
$$


by Lemma 7.6. It can be checked also directly: The left hand side is the dimension of the Coulomb branch for $(\underline{\mathbf{v}}, \underline{\mathbf{w}})$. The right hand side is the dimension of the Higgs branch for $\left(\underline{\mathbf{v}}^{\prime}, \underline{\mathbf{w}}^{\prime}\right)$.

Remark 7.12 applies also to this case: We have an isomorphism $\mathcal{M}_{\nu}(\underline{\mathbf{v}}, \underline{\mathbf{w}}) \cong \mathfrak{M}_{\nu}\left(\underline{\mathbf{v}}^{\prime}, \underline{\mathbf{w}}^{\prime}\right)$ for any parameter $\nu$.

Remark 7.24. As mentioned in Introduction, Corollary 7.21 gives a proof of the conjecture that the Coulomb branch $\mathcal{M}_{C}(\underline{\mathbf{v}}, \underline{\mathbf{w}})$ is a quiver variety of an affine type $A_{\ell-1}$ introduced in [Nak94b] when $\sum_{i} \mathbf{w}_{i} \Lambda_{i}-\mathbf{v}_{i} \alpha_{i}$ is dominant [Nak16]. Its dimension vectors are given by the same way as above up to a diagram automorphism. It is compatible with the level rank duality and the conjectural geometric Satake correspondence for affine Kac-Moody groups [BF10]. It is because the conjecture was based on [Nak09], where a proposal of [BF10] was checked for type $A$ using the level rank duality.

Remark 7.25. Consider a bow diagram of finite type $A_{n-1}$. We consider it as affine type $A_{n-1}$ with $\operatorname{dim} V_{\zeta^{-}}=0=\operatorname{dim} V_{\zeta^{+}}$where $\zeta^{-} \rightarrow 0 \rightarrow \zeta^{+}$, e.g., $\mathbf{v}_{0}=0=\mathbf{v}_{n-1}^{\prime}$ in Figure 2. Then the above results can be applied to a finite type bow diagram:

- In Lemma 7.18, the condition $N\left(x_{0}, x_{1}\right) \geq 0$ is always satisfied as $N\left(x_{0}, x_{1}\right)=\mathbf{v}_{1}+\mathbf{v}_{n-1}$. Also $N\left(x_{n-1}, x_{0}\right)=\mathbf{v}_{n-2}+\mathbf{w}_{n-1}-2 \mathbf{v}_{n-1}=\mathbf{u}_{n-1}$. Therefore $N\left(x_{n-1}, x_{0}\right) \geq 0$ is a part of the dominance condition.

- As for Proposition 7.20, we perform the movement of $\bigcirc$ in the proof in the finite part, that is $\mathrm{O}$ does not cross $x_{0}$. Then the condition $\mathbf{v}_{0}=0=\mathbf{v}_{n-1}^{\prime}$ is preserved. Moreover, $\mathrm{O}$ remained between $x_{0}$ and $x_{n-1}$ are irrelevant: Since $\mathbf{v}_{n-1}^{\prime}=0$ and the cobalanced condition is satisfied, dimensions between $x_{0}$ and $x_{n-1}$ are all 0 . In particular $\mathbf{v}_{n-1}=0$, and we can remove $\mathrm{O}$.

\section{7 $\quad$ Stratification for affine type $A$}

Now we determine the stratification (4.3) for the affine case. Consider the Coulomb branch of the framed quiver gauge theory for dimension vectors $\underline{\mathbf{v}}, \underline{\mathbf{w}}$. We assign $\lambda:=\sum_{i=0}^{n-1} \mathbf{w}_{i} \Lambda_{i}+\mathbf{v}_{0} \delta$, $\mu:=\sum_{i=0}^{n-1} \mathbf{w}_{i} \Lambda_{i}-\mathbf{v}_{i} \alpha_{i}+\mathbf{v}_{0} \delta$ as before. We have a similar result as in finite case.

Theorem 7.26. (1) Suppose $\ell \neq 1$. We have a stratification

$$
\mathcal{M}(\mu, \lambda)=\bigsqcup_{\kappa, \underline{k}} \mathcal{M}^{\mathrm{s}}(\mu, \kappa) \times S^{\underline{k}}\left(\mathbb{C}^{2} \backslash\{0\} /(\mathbb{Z} / \ell \mathbb{Z})\right),
$$

where $\underline{k}=\left[k_{1}, k_{2}, \ldots\right]$ is a partition, and $\kappa$ is a dominant weight between $\mu$ and $\lambda-|\underline{k}| \delta$. We have

$$
\mathcal{M}^{\mathrm{s}}(\mu, \kappa) \begin{cases}\neq \emptyset & \text { if } n \neq 1 \text { or } \mu=\kappa, \\ =\emptyset & \text { if } n=1 \text { and } \mu \neq \kappa .\end{cases}
$$

Moreover a transversal slice to $\mathcal{M}^{\mathrm{s}}(\mu, \kappa) \times S^{k}\left(\mathbb{C}^{2} \backslash\{0\} /(\mathbb{Z} / \ell \mathbb{Z})\right)$ in $\mathcal{M}(\mu, \lambda)$ is isomorphic to $\mathcal{M}(\kappa, \lambda-|\underline{k}| \delta) \times{ }^{c} \mathcal{U}_{n}^{k_{1}} \times{ }^{c} \mathcal{U}_{n}^{k_{2}} \times \cdots$.

(2) Suppose $\ell=1$. The same is true if we replace $\mathbb{C}^{2} \backslash\{0\} /(\mathbb{Z} / \ell \mathbb{Z})$ by $\mathbb{C}^{2}$ and we only allow $\kappa=\lambda-|\underline{k}| \delta$.

Here ${ }^{c} \mathcal{U}_{n}^{k}$ denotes the centered Uhlenbeck space for rank $n$, instanton charge $k$. In other words, it is the factor of the quiver variety $\mathfrak{M}_{0}(k, n)$ associated with the Jordan quiver with dimension vectors $(k, n)$ such that $\mathfrak{M}_{0}(k, n)={ }^{c} \mathcal{U}_{n}^{k} \times \mathbb{A}^{2}$.

Proof. The proof goes as in Theorem 7.13, but we cannot use nilpotent orbits, so we will use quiver varieties instead.

We start with a bow diagram with the balanced condition, and apply Hanany-Witten transition to arrive at (7.23). Let us consider the stratification (4.3) for (7.23). Since $\nu=0, \underline{\mathbf{v}}^{\alpha}$ is a coordinate 
vector of the affine Dynkin diagram of type $A_{\ell-1}$. For segments between $h_{1}$ and $h_{2}, \ldots, h_{\ell-1}$ and $h_{\ell}, \mathfrak{M}^{\mathrm{s}}\left(\underline{\mathbf{v}}^{\alpha}\right)$ is just given by one dimension vector space on the segment and 0 elsewhere (and hence linear maps are all 0$)$. However we have $\times$ between $h_{\ell}$ and $h_{1}$, hence the corresponding $\mathfrak{M}^{\mathrm{s}}\left(\underline{\mathbf{v}}^{\alpha}\right)$ is given by the data for $\mathbb{C}$ on all segments between $h_{1}$ and $h_{\ell}$ through $x_{1}, x_{2}, \ldots, x_{0}$. Therefore the remaining vector $\underline{\mathbf{v}}^{\prime}$ has the following form:

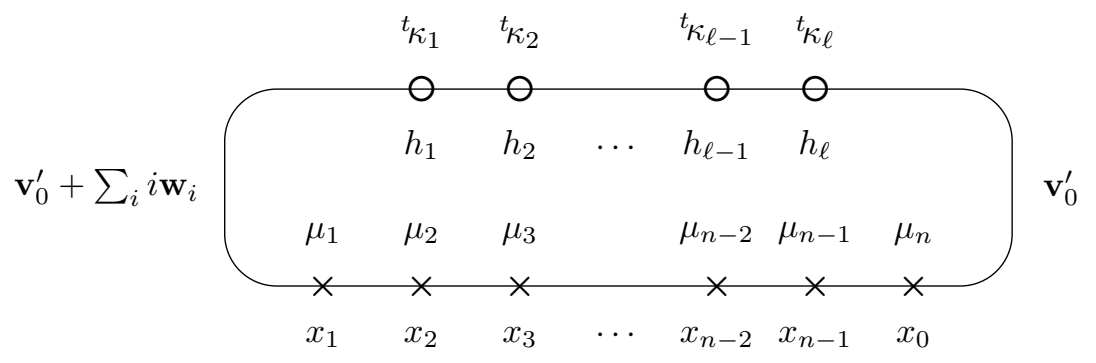

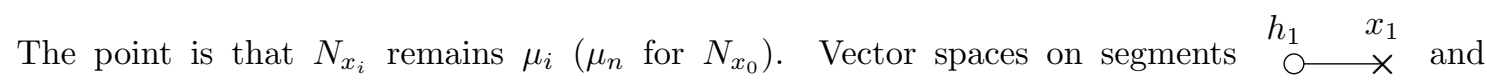
$\begin{array}{cc}x_{0} & h_{\ell} \\ \times & \bigcirc\end{array}$ decrease their dimensions in the same amount, hence they are $\mathbf{v}_{0}^{\prime}$ and $\mathbf{v}_{0}^{\prime}+\sum i \mathbf{w}_{i}$. There is a constraint $\left|{ }^{t} \kappa\right|:=\sum^{t} \kappa_{\sigma}=|\mu|$. In fact, starting from $\mathbf{v}_{0}^{\prime}$, calculate dimensions of vector spaces going the lower and upper halves. Two answers must match at $\mathbf{v}_{0}^{\prime}+\sum i \mathbf{w}_{i}$.

Claim. $\left[{ }^{t} \kappa_{1}, \ldots,{ }^{t} \kappa_{\ell}\right]$ is a generalized Young diagram with the level $n$ constraint.

We consider the upper half as a quiver variety as in the proof of Theorem 7.13. By the necessary condition for $\mathfrak{M}_{0}^{\text {reg }} \neq \emptyset$ in [Nak94b, Lem. 8.1], [Nak98, Lem. 4.7], we have ${ }^{t} \kappa_{1} \geq{ }^{t} \kappa_{2} \geq \cdots \geq{ }^{t} \kappa_{\ell}$. Next we move $h_{\ell}$ to the left of $h_{1}$ by Hanany-Witten transitions going the lower half. Then ${ }^{t} \kappa_{\ell}$ is changed to ${ }^{t} \kappa_{\ell}+n$, as $N\left(h_{\ell}, h_{1}\right)$ must be preserved. Then the necessary condition says ${ }^{t} \kappa_{\ell}+n \geq{ }^{t} \kappa_{1}$, which is nothing but the level $n$ constraint. The claim is proved.

Hence we have $N\left(h_{\sigma}, h_{\sigma+1}\right), N\left(h_{\ell}, h_{1}\right) \geq 0$, thus corresponding to a unique balanced bow diagram by Proposition 7.20. Thus we have a pair $(\kappa, \mu)$ of $\mathfrak{g l}(n)$ aff-weights such that $\kappa$ is dominant and $\kappa \geq \mu$. Note that $\mu$ does not change even as a $\mathfrak{g l}(n)_{\text {aff-weight thanks to our convention }}$ $\langle d, \mu\rangle=0$. The associated generalized Young diagram $\left[\kappa_{1}, \ldots, \kappa_{n}\right]$ with the level $\ell$ constraint is given by the transpose of the above vector $\left[{ }^{t} \kappa_{1}, \ldots,{ }^{t} \kappa_{\ell}\right]$. We have $|\kappa|=\left|{ }^{t} \kappa\right|=|\mu|$. We also have $\langle d, \kappa\rangle=\mathbf{v}_{0}^{\prime}$.

Since entries of $\underline{\mathbf{v}}-|\underline{k}|(1,1, \ldots, 1)-\underline{\mathbf{v}}^{\prime}$ in (4.3) are nonnegative, we have

$\mathbf{v}_{0}^{\prime} \leq \mathbf{v}_{0}-|\underline{k}|, \quad \mathbf{v}_{0}^{\prime}+{ }^{t} \kappa_{\ell} \leq \mathbf{v}_{0}-|\underline{k}|+{ }^{t} \lambda_{\ell}, \quad \ldots, \quad \mathbf{v}_{0}^{\prime}+{ }^{t} \kappa_{1}+\cdots+{ }^{t} \kappa_{\ell} \leq \mathbf{v}_{0}-|\underline{k}|+{ }^{t} \lambda_{1}+\cdots+{ }^{t} \lambda_{\ell}$.

This is equivalent to $\kappa \leq \lambda-|\underline{k}| \delta$.

Let us study the nonemptyness of $\mathcal{M}^{\mathrm{s}}(\mu, \kappa)$. The argument is almost the same as for finite type, but we need to consider an extra factor $S^{\underline{k}}\left(\mathbb{C}^{2} \backslash\{0\} /(\mathbb{Z} / \ell \mathbb{Z})\right)$ or $S^{\underline{k}}\left(\mathbb{C}^{2}\right)$. It has dimension $2 l(\underline{k})$. On the other hand, if we remove $|\underline{k}| \delta$ from $\kappa$, the dimension of $\mathcal{M}^{\mathrm{s}}(\mu, \kappa)$ drops by $2|\underline{k}| n$. Therefore the extra factor is smaller dimensional if $n \neq 1$, and the same argument as the finite case works.

Next suppose $n=1$. The balanced condition implies the cobalanced one in this case. Therefore $\mathcal{M}(\mu, \kappa)$ is a quiver variety of affine type $A_{\ell-1}$ with level 1. By [Nak99, Prop. 2.10], we have $a=b=0$ automatically, hence $\mathcal{M}^{\mathrm{s}}(\mu, \kappa)=\emptyset$ unless $\mu=\kappa$. (One can also use the criterion in [CB01].)

Finally the description of the transversal slice follows from Proposition 4.4.

Remark 7.27. The same argument shows that arbitrary bow variety not necessarily satisfying the balanced condition has a stratification of the same form. In fact, we move to (7.23) in the beginning of the proof, which is possible without assuming $\lambda$ is dominant. In the remaining argument, we consider $\kappa$ instead of $\lambda$, and the dominance of $\lambda$ is not used. In particular, any bow variety is homeomorphic to one with the balanced condition. 


\subsection{Torus fixed points}

Let us consider the fixed point set of the $\left(\mathbb{C}^{\times}\right)^{n}$-action introduced in $\$ 6.9 .2$. We do not consider its scheme theoretic structure, and are interested only in the underlying topological space.

For study of quiver varieties, it is useful to analyze torus fixed points. See e.g., [Nak94a]. However situation for bow varieties is different as the following analysis indicates.

Let us start with an example.

Example 7.28. Consider the bow variety of type $A_{1}$ with $\operatorname{dimension}$ vectors $\left(\mathbf{v}_{1}, \mathbf{w}_{1}\right)$ with $\mathbf{w}_{1}=1$ :

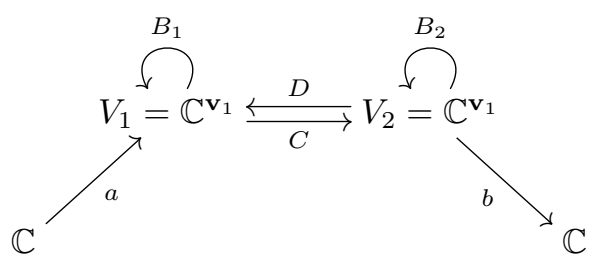

The $\mathbb{C}^{\times}$-action is given by $b \mapsto t b$ and other maps are preserved. There is no possibility of the second summand in Lemma 4.1 , hence $\mathcal{M}=\mathcal{M}^{\mathrm{s}}$. Then a $\mathbb{C}^{\times}$-fixed point has a representative $\left(B_{1}, B_{2}, a, b, C, D\right)$ such that there are 1-parameter subgroups $\rho_{1}: \mathbb{C}^{\times} \rightarrow \mathrm{GL}\left(V_{1}\right), \rho_{2}: \mathbb{C}^{\times} \rightarrow$ $\mathrm{GL}\left(V_{2}\right)$ such that

$$
\left(B_{1}, B_{2}, a, t b, C, D\right)=\left(\rho_{1}(t) B_{1} \rho_{1}(t)^{-1}, \rho_{2}(t) B_{2} \rho_{2}(t)^{-1}, \rho_{1}(t) a, b \rho_{2}(t)^{-1}, \rho_{2}(t) C \rho_{1}(t)^{-1}, \rho_{1}(t) D \rho_{2}(t)^{-1}\right) .
$$

We decompose $V_{1}=\bigoplus V_{1}(m), V_{2}=\bigoplus V_{2}(m)$ into weight spaces. Then $\operatorname{Im} a \subset V_{1}(0), B_{1}\left(V_{1}(m)\right) \subset$ $V_{1}(m)$. By (S2) we must have $V_{1}=V_{1}(0)$. Similarly $V_{2}=V_{2}(-1)$. Then we must have $C=0=D$, hence $B_{1}=D C=0, B_{2}=C D=0$. Therefore (S1) and (S2) imply $\mathbf{v}_{1}=1$. In other words, $\mathcal{M}^{\mathbb{C}^{\times}}$ is a single point if $\mathbf{v}_{1}=1$, and is empty if $\mathbf{v}_{1}>1$.

This is compatible with Hikita conjecture [Hik17] (see also [Nak16, $§ 1(v i i i)]$ ): the cohomology ring of Higgs branch with generic moment map level is isomorphic to the coordinate ring of the torus fixed point subscheme. In this example, Higgs branch is a quiver variety of type $A_{1}$ with dimension vectors $\left(\mathbf{v}_{1}, \mathbf{w}_{1}=1\right)$. It is a single point if $\mathbf{v}_{1}=1$, and is empty if $\mathbf{v}_{1}>1$.

Note that the coordinate ring of the empty set is the 0 -dimensional vector space. And the cohomology ring of the empty set is also the 0-dimensional vector space.

On the other hand, it is not clear how to formulate Hikita conjecture when Higgs and Coulomb branches are swapped: Suppose $\mathbf{v}_{1}>1$. Since $\mathcal{M}=\mathcal{M}^{\mathrm{s}}$, it cannot be changed by the perturbation of the stability condition. (Any symplectic resolution must be $\mathcal{M}$ itself.) Since the Euler number of $\mathcal{M}$ is 0 , the cohomology group of $\mathcal{M}$ is nontrivial at least in two degrees, the degree 0 and an odd degree. In the usual formulation, we implicitly assume there is no odd cohomology group, as the coordinate ring side is always commutative, not super commutative. Also, a naive definition of the corresponding Higgs branch is just the categorical quotient $\mathfrak{M}_{0}(\underline{\mathbf{v}}, \underline{\mathbf{w}})$, which is a single point $\{0\}$ in the reduced structure.

Let us consider the case $\underline{\mathbf{w}}=0$. Let us denote $\left(\mathbb{C}^{\times}\right)^{n}$ by $T$ hereafter.

Proposition 7.29. The fixed point set $\mathcal{M}(\underline{\mathbf{v}}, 0)^{T}$ is empty unless $\underline{\mathbf{v}}=0$.

Proof. Consider the stratification in Proposition 6.5(3). Let us first consider the factor $S^{k}\left(\mathbb{C} \times \mathbb{C}^{\times}\right)$. The action of $\left(t_{0}, \ldots, t_{n-1}\right)$ is trivial on the factor. The extra $\mathbb{C}^{\times}$acts on $S^{k}\left(\mathbb{C} \times \mathbb{C}^{\times}\right)$by the multiplication on $\mathbb{C}^{\times}$by its definition. Therefore we do not have fixed points in $S^{k}\left(\mathbb{C} \times \mathbb{C}^{\times}\right)$unless $k=0$.

Next consider $\mathcal{M}^{\mathrm{reg}}(\underline{\mathbf{v}}, 0)$. Let us take a representative $\left(A_{i}, B_{i}, a_{i}, b_{i}\right)(0 \leq i \leq n-1)$ of a fixed point. We have a (unique) homomorphism $\rho: T \rightarrow \prod \mathrm{GL}\left(V_{i}\right)$ such that

$$
\begin{gathered}
t_{\delta} A_{n-1}=\rho_{0}(t) A_{n-1} \rho_{n-1}(t)^{-1}, \quad A_{i}=\rho_{i+1}(t) A_{i} \rho_{i}(t)^{-1}(i \neq n-1), \quad B_{i}=\rho_{i}(t) B_{i} \rho_{i}(t)^{-1}, \\
t_{i}^{-1} a_{i}=\rho_{i}(t) a_{i}, \quad t_{\delta} t_{0} b_{n-1}=b_{n-1} \rho_{n-1}(t)^{-1}, \quad t_{i+1} b_{i}=b_{i} \rho_{i}(t)^{-1}(i \neq n-1),
\end{gathered}
$$


where $\rho_{i}(t)$ is the $\mathrm{GL}\left(V_{i}\right)$-component of $\rho$.

We consider the weight space decomposition of $V_{i}$ with respect to $\rho_{i}$. By the first equation, $A_{n-1}$ and $b_{n-1}$ increase weights for $t_{\delta}$ by 1 , and other maps preserve them. (We understand that $W_{i}$ has weight 0 .) Therefore we can unwind the chainsaw quiver to a handsaw quiver as

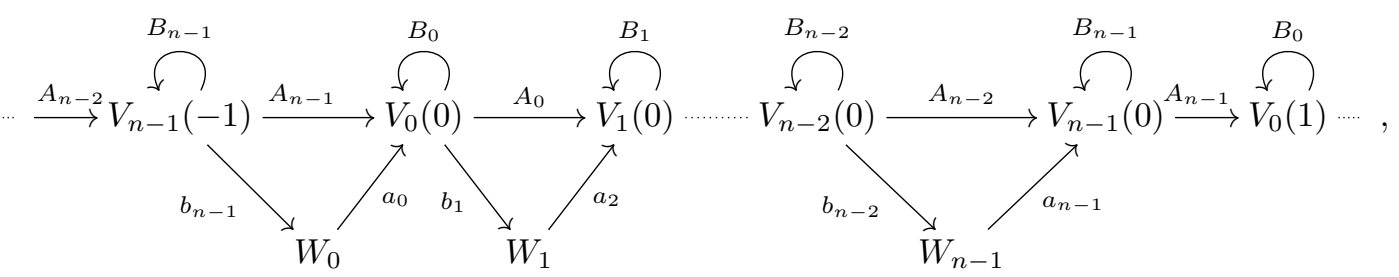

where the number in ( ) is the $t_{\delta}$-weight. For a triangle without $W$, we have $B_{j} A_{j-1}=A_{j-1} B_{j-1}$. Then the conditions (S1),(S2) imply that $A_{j-1}$ is an isomorphism. But $V_{j}(m)=0$ if $|m|$ is sufficiently large. Therefore $V_{j}(m)=0$ if $m \neq 0$ or $j=n-1, m=0$.

Next consider the action of $\left(t_{0}, \ldots, t_{n-1}\right)$. The representative is a direct sum of $n$ summands, $\left(a_{j}, b_{j-1}\right)$ is zero except possibly for $j=i(0 \leq i \leq n-1)$. If $a_{j}=0=b_{j-1}$, we have $A_{j-1}$ is an isomorphism as above. Then $V_{n-1}(-1)=0=V_{n-1}(0)$ imply any $V_{i}(0)$ also vanishes.

Next consider a general case.

Proposition 7.30. Suppose $\nu=0$. The fixed point set $\mathcal{M}(\mu, \lambda)^{T}$ is either a single point or empty.

Proof. We prove $\mathcal{M}^{\mathrm{s}}(\mu, \kappa)^{T}$ is a single point with a specific $\kappa$ determined by $\mu$, and is $\emptyset$ otherwise.

As in the proof of Proposition 7.29, we choose a representative of a fixed point and take a homomorphism $\rho: T \rightarrow \prod \mathrm{GL}\left(V_{\zeta}\right)$. We unwind the bow diagram as above, and have a direct sum decomposition into $n$ summands, where $\left(a_{j}, b_{j-1}\right)$ is zero except possibly for $j=i(0 \leq i \leq n-1)$ as above. Since $A_{j-1}$ is an isomorphism if $j \neq i$, we can remove the corresponding segment from the bow diagram so that the corresponding bow variety does not change. Therefore the $i$ th summand corresponds to

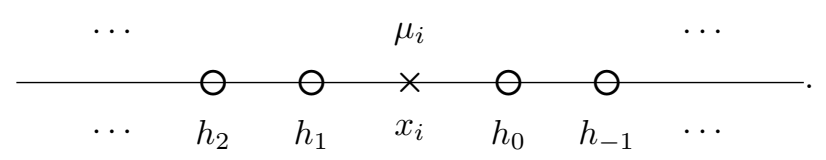

Note that $N_{x_{i}}=\mu_{i}$ as $A_{i-1}$ is an isomorphism for other summands. Let us number $\bigcirc$ as above.

As in the proof of Theorem 7.26, we have $N\left(h_{\sigma}, h_{\sigma+1}\right) \geq 0$ for any $\sigma$ by the necessary condition for $\mathfrak{M}_{0}^{\text {reg }} \neq \emptyset$. On the other hand, $\sum N\left(h_{\sigma}, h_{\sigma+1}\right)=1$ by definition. Therefore $N\left(h_{\sigma}, h_{\sigma+1}\right)=1$ for some $\sigma_{0}$ and $=0$ otherwise. Suppose $\sigma_{0}>0$. Then $N\left(h_{\sigma}, h_{\sigma+1}\right)=0$ for $\sigma<\sigma_{0}$. Consider vector spaces on segments which are right of $x_{i}$. As $N_{h_{\sigma}}=0$ for sufficiently small $\sigma$, we have $N_{h_{\sigma}}=0$ for $\sigma \leq 0$. Thus vector spaces which are right of $x_{i}$ vanish. Since $N\left(h_{0}, h_{1}\right)$ is also 0 by assumption, we have $N_{h_{1}}=1$. Moving to the left of $x_{i}$, we get $N_{h_{1}}=N_{h_{2}}=\cdots=N_{h_{\sigma_{0}}}=1$, $N_{h_{\sigma_{0}+1}}=N_{h_{\sigma_{0}+2}}=\cdots=0$. Hence the vector space on the segment $\left[h_{\sigma_{0}}, h_{\sigma_{0}-1}\right]$ is 1-dimensional, the next one on $\left[h_{\sigma_{0}-1}, h_{\sigma_{0}-2}\right]$ is 2-dimensional, and so on. The vector space over $\left[h_{1}, x_{i}\right]$ is $\sigma_{0^{-}}$ dimensional. In particular, $\sigma_{0}=\mu_{i}$. The corresponding bow variety is isomorphic to $\overline{\mathcal{N}}(\lambda) \cap \mathcal{S}(\mu)$ with $\lambda=\left(\sigma_{0}\right),{ }^{t} \mu=\left(1^{\sigma_{0}}\right)$ by the argument in $\S 7.4$. Then $\lambda=\mu$, hence it is a single point.

Similarly $\sigma_{0}<0$ implies $-1=N_{h_{0}}=N_{h_{-1}}=\cdots=N_{h_{\sigma_{0}+1}}, 0=N_{h_{\sigma_{0}}}=N_{h_{\sigma_{0}}-1}=\cdots$. We get $\sigma_{0}=\mu_{i}$ also in this case. The corresponding bow variety is again a single point. If $\sigma_{0}=0$, all vector spaces vanish, and $\mu_{i}=0=\sigma_{0}$.

Note that dimension of vector spaces are given by $\mu_{i}$, hence $\kappa$ is determined by $\mu$.

According to Hikita conjecture mentioned above, we expect $\mathcal{M}(\mu, \lambda)^{T} \neq \emptyset$ if and only if the corresponding quiver variety $\mathfrak{M}(\mu, \lambda)$ is nonempty with generic moment map level. The latter is equivalent that the weight space $L(\lambda)_{\mu}$ is nonzero, where $L(\lambda)$ is the integrable representation of the highest weight $\lambda$. 
Proposition 7.32. Suppose $\nu$ is arbitrary. The fixed point set $\mathcal{M}(\mu, \lambda)^{T}$ is finite.

Proof. We argue as in Proposition 7.30. We have a direct sum decomposition of a representative of a fixed point as in (7.31).

According to the sign of $\mu_{i}$, we apply Hanany-Witten transitions $\left|\mu_{i}\right|$ times to move $\times$ to the left or right. We claim that it is possible, i.e., the number of $\bigcirc$ to the left or right of $x_{i}$ is more than or equal to $\left|\mu_{i}\right|$. In fact, if this is not possible, the last step is

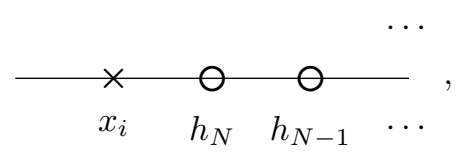

when $\mu_{i}>0$. Since the dimension on the left segment to $x_{i}$ is $0, N_{x_{i}}>0$ means the dimension on the right segment is negative. This is a contradiction. The same is true if $\mu_{i}<0$.

Thus the cobalanced condition is met by Hanany-Witten transitions, hence the bow variety containing the direct summand is isomorphic to a quiver variety of type $A$. Since the framed vector space $W$ has dimension 1, the quiver variety is a single point.

We have only finitely many ways to decompose dimension vectors, the fixed point set is finite.

Let us return to the case $\nu=0$ so that $\mathcal{M}(\mu, \lambda)$ is affine. We further assume $\mathcal{M}(\mu, \lambda)^{T} \neq \emptyset$, so $\mathcal{M}(\mu, \lambda)^{T}$ is a single point by Proposition 7.30. Take a generic 1-parameter subgroup $\rho: \mathbb{C}^{\times} \rightarrow T$ such that $\mathcal{M}(\mu, \lambda)^{\rho\left(\mathbb{C}^{\times}\right)}=\mathcal{M}(\mu, \lambda)^{T}$. We have a diagram

$$
\mathrm{pt}=\mathcal{M}(\mu, \lambda)^{T} \stackrel{p}{\leftarrow} \mathcal{A} \stackrel{j}{\rightarrow} \mathcal{M}(\mu, \lambda),
$$

where $\mathcal{A}$ is the attracting set consisting of points $x$ such that $\lim _{t \rightarrow 0} \rho(t) x$ exists. The map $p$ is given by the limit, and $j$ is the inclusion. We have Braden's hyperbolic restriction functor $p_{*} j^{!}$on the $T$-equivariant derived category of constructible sheaves on $\mathcal{M}(\mu, \lambda)$ [Bra03]. Let $\operatorname{IC}(\mathcal{M}(\mu, \lambda))$ be the intersection cohomology complex associated with the trivial local system on $\mathcal{M}(\mu, \lambda)$. We consider $p_{*} j$ ! $\operatorname{IC}(\mathcal{M}(\mu, \lambda))$. In [BFN16c, §3] the notion of hyperbolic semi-smallness is introduced, which implies that $p_{*} j^{!} \mathrm{IC}(\mathcal{M}(\mu, \lambda))$ is a vector space (instead of complex of vector spaces).

Proposition 7.33. $p_{*} j^{!}$is hyperbolic semi-small. Therefore $p_{*} j^{!} \operatorname{IC}(\mathcal{M}(\mu, \lambda))$ is a vector space.

Proof. Let us assume $\ell \neq 1$ for notational simplicity.

Recall the stratification $\mathcal{M}(\mu, \lambda)=\bigsqcup \mathcal{M}^{\mathrm{s}}(\mu, \kappa) \times S^{\underline{k}}\left(\mathbb{C}^{2} \backslash\{0\} /(\mathbb{Z} / \ell \mathbb{Z})\right)$ in Theorem 7.26. Then $\operatorname{IC}(\mathcal{M}(\mu, \lambda))$ is a locally constant sheaf up to shifts on each stratum. The hyperbolic semi-smallness in this case is

$$
\operatorname{dim} \mathcal{A} \cap X_{\kappa, \underline{k}} \leq \frac{1}{2} \operatorname{dim} X_{\kappa, \underline{k}}, \quad X_{\kappa, \underline{k}}:=\mathcal{M}^{\mathrm{s}}(\mu, \kappa) \times S^{\underline{k}}\left(\mathbb{C}^{2} \backslash\{0\} /(\mathbb{Z} / \ell \mathbb{Z})\right),
$$

and also the same estimate holds for the repelling set $\mathcal{R}=\left\{x \mid \lim _{t \rightarrow \infty} \rho(t) x\right.$ exists $\}$.

On the factor $S^{\underline{k}}\left(\mathbb{C}^{2} \backslash\{0\} /(\mathbb{Z} / \ell \mathbb{Z})\right)$, the $T$-action is induced from $t \cdot\left(z_{1}, z_{2}\right)=\left(t z_{1}, t^{-1} z_{2}\right)$ for $\left(z_{1}, z_{2}\right) \in \mathbb{C}^{2}$. Therefore the attracting and repelling sets are $z_{2}=0$ and $z_{1}=0$ respectively. They are half-dimensional in $\mathbb{C}^{2}$. Hence we may assume $\underline{k}=\emptyset$.

We take a generic parameter $\nu^{\mathbb{R}}$ so that $\pi: \mathcal{M}_{\left(0, \nu^{\mathbb{R}}\right)}(\mu, \kappa) \rightarrow \mathcal{M}(\mu, \kappa)$ is a resolution. Since $\pi^{-1}\left(\mathcal{A} \cap \mathcal{M}^{\mathrm{s}}(\mu, \kappa)\right)$ (resp. $\left.\pi^{-1}\left(\mathcal{R} \cap \mathcal{M}^{\mathrm{s}}(\mu, \kappa)\right)\right)$ is contained in the attracting set $\widetilde{\mathcal{A}}$ (resp. repelling set $\widetilde{\mathcal{R}})$ for $\mathcal{M}_{\left(0, \nu^{\mathbb{R}}\right)}(\mu, \kappa)$, it is enough to estimate the dimension of $\widetilde{\mathcal{A}}$ and $\widetilde{\mathcal{R}}$.

Since $\mathcal{M}_{\left(0, \nu^{\mathbb{R}}\right)}(\mu, \kappa)$ is smooth, we can use the Bialynicki-Birula decomposition. The tangent space at a fixed point set decomposes into weight spaces with respect to $\rho\left(\mathbb{C}^{\times}\right)$and the dimension of $\widetilde{\mathcal{A}}$ (resp. $\widetilde{\mathcal{R}}$ ) is sum of dimensions of positive (resp. negative) weight spaces. Since $T$ preserves the symplectic form, positive and negative weight spaces are dual to each other. Therefore we have $\operatorname{dim} \widetilde{\mathcal{A}}=\operatorname{dim} \widetilde{\mathcal{R}}=\frac{1}{2} \operatorname{dim} \mathcal{M}_{\left(0, \nu^{\mathbb{R}}\right)}(\mu, \kappa)$. 
We have a base of $p_{*} j^{\prime} \operatorname{IC}(\mathcal{M}(\mu, \lambda))$ given by $\frac{1}{2} \operatorname{dim} \mathcal{M}(\mu, \lambda)$-dimensional irreducible components of $\mathcal{A}$. (See [BFN16c, Th. 3.23].)

It is expected that the hyperbolic restriction functor and the pushforward by the semi-small resolution are 'dual' in Higgs and Coulomb branches. Therefore

Conjecture 7.34. $\operatorname{dim} p_{*} j^{!} \operatorname{IC}(\mathcal{M}(\mu, \lambda))=\operatorname{dim} L(\lambda)_{\mu}$.

If $\mu$ is dominant, $\mathcal{M}(\mu, \lambda)$ is a quiver variety by Corollary 7.21. Then the attracting set is the so-called tensor product variety. In particular, the number of irreducible components is given by a tensor product multiplicity $([\mathrm{Nak} 09, \S 6])$. The conjecture follows from the level-rank duality. Recall that [Nak09] is motivated by the conjectural geometric Satake correspondence for affine Kac-Moody groups [BF10]. The above conjecture is a refinement of the geometric Satake correspondence, as $\mu$ is not necessarily dominant, or it could be even not a weight of $L(\lambda)$, if we understand $\operatorname{dim} L(\lambda)_{\mu}$ as 0 . See also [BF13] for another (closely related but different) conjectural geometric realization of $L(\lambda)_{\mu}$ for arbitrary $\mu$.

Recall also irreducible components of $\mathcal{A}$ for dominant $\mu$ are regarded as Mirković-Vilonen (MV) cycles for affine Kac-Moody groups of type $A$ in $[N a k 09, \S 6]$. We now remove the assumption that $\mu$ is dominant, hence we get MV cycles for arbitrary weights. It is natural to expect

Conjecture 7.35. The union of sets of $\frac{1}{2} \operatorname{dim} \mathcal{M}(\mu, \lambda)$-dimensional irreducible components of $\mathcal{A}$ has a structure of a crystal, isomorphic to the crystal of the integrable highest weight representation $L(\lambda)$.

\section{Appendix}

\section{A Symplectic structures under Hanany-Witten transition}

In this section, we describe Hanany-Witten transition more explicitly in terms of matrices, and show that symplectic structures are preserved under Hanany-Witten transition.

We consider four cases depending on the relation between $m$ and $n$.

(i) $m=n$

(ii) $m=n+1$

(iii) $m<n$

(iv) $m>n+1$

Now, when $m \leq n$, we have $m^{\prime} \geq l+1$, and we can see that the assertions (i) and (ii), and (iii) and (iv) are equivalent respectively:

$$
\begin{aligned}
& (m \leq n) \stackrel{l}{{ }^{\prime m}}{ }^{n} \cong \stackrel{l}{\times} \cong m^{\prime} \bigcirc^{n}\left(m^{\prime} \geq l+1\right) \\
& \Longleftrightarrow\left(m^{\prime} \geq l+1\right) \stackrel{n}{{ }^{m^{\prime}} \times l} \cong \stackrel{n}{\times^{m}} \cong{ }^{l} \quad(m \leq n)
\end{aligned}
$$

Thus it is enough to show the assertion (i) and (iii).

(i) Let us consider the symplectic reduction by $\mathrm{GL}\left(V_{2}\right)$ of the first space in the proof of Proposition 7.1. Recall that the triangle part $\widetilde{\mathcal{M}}$ is $T^{*} \mathrm{GL}\left(V_{2}\right) \times \operatorname{Hom}\left(\mathbb{C}, V_{3}\right) \times \operatorname{Hom}\left(V_{2}, \mathbb{C}\right)$ by Proposition 3.2 as $\operatorname{dim} V_{2}=\operatorname{dim} V_{3}$. Then the symplectic reduction is

$$
\operatorname{Hom}\left(V_{1}, V_{3}\right) \times \operatorname{Hom}\left(V_{3}, V_{1}\right) \times \operatorname{Hom}\left(V_{1}, \mathbb{C}\right) \times \operatorname{Hom}\left(\mathbb{C}, V_{1}\right)
$$

by setting

$$
\tilde{C} \stackrel{\text { def. }}{=} A C, \quad \tilde{D} \stackrel{\text { def. }}{=} D A^{-1}, \quad \tilde{a}=a, \quad \tilde{b} \stackrel{\text { def. }}{=} b A^{-1} .
$$

The symplectic form is given by

$$
\operatorname{tr}(d \tilde{C} \wedge d \tilde{D})+\operatorname{tr}(d \tilde{a} \wedge d \tilde{b})
$$


On the other hand, consider the second space in the proof of Proposition 7.1. Then [ $\left.A^{\text {new }} a^{\text {new }}\right]: V_{1} \oplus$ $\mathbb{C} \rightarrow V_{2}^{\text {new }}$ is an isomorphism, and the symplectic reduction by $\mathrm{GL}\left(V^{\text {new }}\right)$ is

$$
\operatorname{Hom}\left(V_{1} \oplus \mathbb{C}, V_{3}\right) \times \operatorname{Hom}\left(V_{3}, V_{1} \oplus \mathbb{C}\right)
$$

by setting

$$
\tilde{C}^{\text {new }} \stackrel{\text { def. }}{=} C^{\text {new }}\left[\begin{array}{ll}
A^{\text {new }} & a^{\text {new }}
\end{array}\right], \quad \tilde{D}^{\text {new }} \stackrel{\text { def. }}{=}\left[\begin{array}{ll}
A^{\text {new }} & a^{\text {new }}
\end{array}\right]^{-1} D^{\text {new }} .
$$

The symplectic form is given by

$$
\operatorname{tr}\left(d \tilde{C}^{\text {new }} \wedge d \tilde{D}^{\text {new }}\right) .
$$

Looking at definitions of Hanany-Witten transition, we find

$$
\tilde{C}^{\text {new }}=\left[\begin{array}{ll}
\tilde{C} & a
\end{array}\right], \quad \tilde{D}^{\text {new }}=\left[\begin{array}{c}
\tilde{D} \\
\tilde{b}
\end{array}\right] .
$$

Therefore the symplectic forms are the same.

Data and Hanany-Witten transition are illustrated as follows:

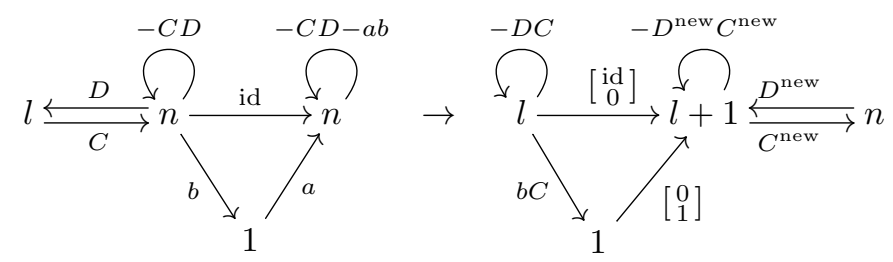

(iii) Let us consider the second space in the proof of Proposition 7.1. By Proposition 3.2 the symplectic reduction by $\mathrm{GL}\left(V_{2}^{\text {new }}\right)=\mathrm{GL}\left(m^{\prime}\right)$ is isomorphic to

$$
\left\{\left(\eta, C^{\text {new }}, D^{\text {new }}\right) \in \mathcal{S}\left(l, m^{\prime}\right) \times \operatorname{Hom}\left(\mathbb{C}^{m^{\prime}}, \mathbb{C}^{n}\right) \times \operatorname{Hom}\left(\mathbb{C}^{n}, \mathbb{C}^{m^{\prime}}\right) \mid \eta=D^{\text {new }} C^{\text {new }}\right\},
$$

where we write dimensions for the slice explicitly. The symplectic form is $\operatorname{tr}\left(d C^{\text {new }} \wedge d D^{\text {new }}\right)$.

Let us consider the first space in the proof of Proposition 7.1. It is the product of $\operatorname{Hom}\left(\mathbb{C}^{l}, \mathbb{C}^{m}\right) \times$ $\operatorname{Hom}\left(\mathbb{C}^{m}, \mathbb{C}^{l}\right)$ and $\operatorname{GL}(n) \times \mathcal{S}(m, n)$ by Proposition 3.2. The symplectic reduction by $\operatorname{GL}(m)$ is described as

$$
\left\{\left(C, D, \eta^{\prime}, u\right) \in \operatorname{Hom}\left(\mathbb{C}^{l}, \mathbb{C}^{m}\right) \times \operatorname{Hom}\left(\mathbb{C}^{m}, \mathbb{C}^{l}\right) \times \mathcal{S}(m, n) \times \operatorname{GL}(n) \mid C D=h^{\prime}\right\} / \mathrm{GL}(m),
$$

where $\eta^{\prime}$ has the block decompositon as

$$
\eta^{\prime}=\left[\begin{array}{ccc}
h^{\prime} & 0 & g \\
f & 0 & e_{0} \\
0 & \text { id } & e
\end{array}\right]
$$

The symplectic form is $\operatorname{tr}\left(d \eta^{\prime} \wedge d u u^{-1}+\eta^{\prime} d u u^{-1} \wedge d u u^{-1}\right)+\operatorname{tr}(d C \wedge d D)$. See

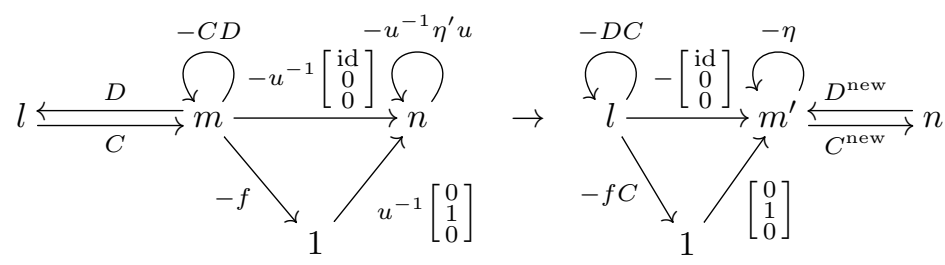

The decomposition $\mathbb{C}^{n}=\mathbb{C}^{m} \oplus \mathbb{C}^{n-m-1} \oplus \mathbb{C}$ in the Hurtubise normal form induces an isomorphism $V_{2}^{\text {new }} \cong \mathbb{C}^{l} \oplus \mathbb{C}^{n-m} \oplus \mathbb{C}$. Then we find

$$
C^{\text {new }}=u^{-1}\left[\begin{array}{ccc}
C & 0 & g \\
0 & \text { id } & e^{+}
\end{array}\right], \quad D^{\text {new }}=\left[\begin{array}{ll}
D & 0 \\
f & 0 \\
0 & \text { id }
\end{array}\right] u,
$$


where $e^{+}=\left[\begin{array}{c}e_{0} \\ e\end{array}\right]$.

Now

$$
\begin{aligned}
& \operatorname{tr}\left(d C^{\text {new }} \wedge d D^{\text {new }}\right) \\
= & \operatorname{tr}\left(\left(-u^{-1} d u u^{-1}\left[\begin{array}{ccc}
C & 0 & g \\
0 & \text { id } e^{+}
\end{array}\right]+u^{-1}\left[\begin{array}{ccc}
d C & 0 & d g \\
0 & 0 & d e+
\end{array}\right]\right) \wedge\left(\left[\begin{array}{ccc}
d D & 0 \\
d f & 0 \\
0 & 0
\end{array}\right] u+\left[\begin{array}{cc}
D & 0 \\
f & 0 \\
0 & \text { id }
\end{array}\right] d u\right)\right) \\
= & \operatorname{tr}\left(\left[\begin{array}{ccc}
C d D & 0 & 0 \\
d f & 0 & 0 \\
0 & 0 & 0
\end{array}\right] \wedge d u u^{-1}+\eta^{\prime} d u u^{-1} \wedge d u u^{-1}+\left[\begin{array}{ccc}
d C \wedge d D & 0 & 0 \\
0 & 0 & 0 \\
0 & 0 & 0
\end{array}\right]+\left[\begin{array}{ccc}
d C D & 0 & d g \\
0 & 0 & d e_{0} \\
0 & 0 & d e
\end{array}\right] \wedge d u u^{-1}\right) \\
= & \operatorname{tr}\left(d \eta^{\prime} \wedge d u u^{-1}+\eta^{\prime} d u u^{-1} \wedge d u u^{-1}\right)+\operatorname{tr}(d C \wedge d D) .
\end{aligned}
$$

Therefore the symplectic forms are the same.

\section{References}

[BDF14] A. Braverman, G. Dobrovolska, and M. Finkelberg, Gaiotto-Witten superpotential and Whittaker D-modules on monopoles, ArXiv e-prints (2014), arXiv:1406.6671 [math.AG].

[BDG15] M. Bullimore, T. Dimofte, and D. Gaiotto, The Coulomb Branch of $3 d \mathcal{N}=4$ Theories, ArXiv e-prints (2015), arXiv:1503.04817 [hep-th].

[BF10] A. Braverman and M. Finkelberg, Pursuing the double affine Grassmannian I: transversal slices via instantons on $A_{k}$-singularities, Duke Math. J. 152 (2010), no. 2, $175-206$.

[BF13] _ Pursuing the double affine Grassmannian III: Convolution with affine zastava, Mosc. Math. J. 13 (2013), no. 2, 233-265, 363.

[BFN16a] A. Braverman, M. Finkelberg, and H. Nakajima, Towards a mathematical definition of Coulomb branches of 3 -dimensional $\mathcal{N}=4$ gauge theories, II, ArXiv e-prints (2016), arXiv:1601.03586 [math.RT].

[BFN16b] Coulomb branches of $3 d \mathcal{N}=4$ quiver gauge theories and slices in the affine Grassmannian (with appendices by Alexander Braverman, Michael Finkelberg, Joel Kamnitzer, Ryosuke Kodera, Hiraku Nakajima, Ben Webster, and Alex Weekes), ArXiv e-prints (2016), arXiv:1604.03625 [math.RT].

[BFN16c] , Instanton moduli spaces and $\mathcal{W}$-algebras, Astérisque (2016), no. 385, vii+128, arXiv:1406.2381 [math.QA].

[Bie97] R. Bielawski, Hyper-Kähler structures and group actions, J. London Math. Soc. (2) 55 (1997), no. 2, 400-414.

[Bra03] T. Braden, Hyperbolic localization of intersection cohomology, Transform. Groups 8 (2003), no. 3, 209-216.

[BS16] G. Bellamy and T. Schedler, Symplectic resolutions of Quiver varieties and character varieties, ArXiv e-prints (2016), arXiv:1602.00164 [math.AG].

[CB01] W. Crawley-Boevey, Geometry of the moment map for representations of quivers, Compositio Math. 126 (2001), no. 3, 257-293.

[CB03a] Normality of Marsden-Weinstein reductions for representations of quivers, Math. Ann. 325 (2003), no. 1, 55-79.

[CB03b]__ On matrices in prescribed conjugacy classes with no common invariant subspace and sum zero, Duke Math. J. 118 (2003), no. 2, 339-352. 
[Che09] S. A. Cherkis, Moduli spaces of instantons on the Taub-NUT space, Comm. Math. Phys. 290 (2009), no. 2, 719-736.

[Che10] _ Instantons on the Taub-NUT space, Adv. Theor. Math. Phys. 14 (2010), no. $2,609-641$.

[Che11] _ Instantons on gravitons, Comm. Math. Phys. 306 (2011), no. 2, 449-483.

[CK98] S. A. Cherkis and A. Kapustin, Singular monopoles and supersymmetric gauge theories in three dimensions, Nuclear Phys. B 525 (1998), no. 1-2, 215-234.

[COS11] S. A. Cherkis, C. O'Hara, and C. Sämann, Super Yang-Mills theory with impurity walls and instanton moduli spaces, Physical Review D 83 (2011), no. 12, 126009, arXiv:1103.0042 [hep-th].

$\left[\mathrm{dBHO}^{+97]}\right.$ J. de Boer, K. Hori, H. Ooguri, Y. Oz, and Z. Yin, Mirror symmetry in threedimensional gauge theories, $\mathrm{SL}(2, \mathbf{Z})$ and D-brane moduli spaces, Nuclear Phys. B 493 (1997), no. 1-2, 148-176.

[dBHOO97] J. de Boer, K. Hori, H. Ooguri, and Y. Oz, Mirror symmetry in three-dimensional gauge theories, quivers and D-branes, Nuclear Phys. B 493 (1997), no. 1-2, 101-147.

[Don84] S. K. Donaldson, Nahm's equations and the classification of monopoles, Comm. Math. Phys. 96 (1984), no. 3, 387-407.

[FR14] M. Finkelberg and L. Rybnikov, Quantization of Drinfeld zastava in type A, J. Eur. Math. Soc. 16 (2014), no. 2, 235-271.

[Hik17] T. Hikita, An Algebro-Geometric Realization of the Cohomology Ring of Hilbert Scheme of Points in the Affine Plane, Int. Math. Res. Not. IMRN (2017), no. 8, 2538-2561, arXiv:1501.02430 [math.AG].

[Hur89] J. Hurtubise, The classification of monopoles for the classical groups, Comm. Math. Phys. 120 (1989), no. 4, 613-641.

[HW97] A. Hanany and E. Witten, Type IIB superstrings, BPS monopoles, and threedimensional gauge dynamics, Nuclear Phys. B 492 (1997), no. 1-2, 152-190.

[Kin89] A. King, Instantons and holomorphic bundles on the blown up plane, Ph.D. thesis, Oxford, 1989.

[Kin94] A. D. King, Moduli of representations of finite-dimensional algebras, Quart. J. Math. Oxford Ser. (2) 45 (1994), no. 180, 515-530.

[KP79] H. Kraft and C. Procesi, Closures of conjugacy classes of matrices are normal, Invent. Math. 53 (1979), no. 3, 227-247.

[Kro88] P. B. Kronheimer, A hyperkähler structure on the cotangent bundle of a complex manifold, available at http://www. math.harvard.edu/ kronheim, June 1988.

[Kro89] - The construction of ALE spaces as hyper-Kähler quotients, J. Differential Geom. 29 (1989), no. 3, 665-683.

[Kro90] _ Instantons and the geometry of the nilpotent variety, J. Differential Geom. 32 (1990), no. 2, 473-490.

[LBP90] L. Le Bruyn and C. Procesi, Semisimple representations of quivers, Trans. Amer. Math. Soc. 317 (1990), no. 2, 585-598.

[Los06] I. V. Losev, Symplectic slices for actions of reductive groups, Mat. Sb. 197 (2006), no. $2,75-86$. 
[Maf05] A. Maffei, Quiver varieties of type A, Comment. Math. Helv. 80 (2005), no. 1, 1-27.

[Nak94a] H. Nakajima, Homology of moduli spaces of instantons on ALE spaces. I, J. Differential Geom. 40 (1994), no. 1, 105-127.

[Nak94b] _ Instantons on ALE spaces, quiver varieties, and Kac-Moody algebras, Duke Math. J. 76 (1994), no. 2, 365-416.

[Nak98] _ Q Quiver varieties and Kac-Moody algebras, Duke Math. J. 91 (1998), no. 3, 515-560.

[Nak99] L L L Lectures on Hilbert schemes of points on surfaces, University Lecture Series, vol. 18, American Mathematical Society, Providence, RI, 1999.

[Nak01]__ Quiver varieties and finite-dimensional representations of quantum affine algebras, J. Amer. Math. Soc. 14 (2001), no. 1, 145-238 (electronic).

[Nak09] _ Quiver varieties and branching, SIGMA Symmetry Integrability Geom. Methods Appl. 5 (2009), Paper 003, 37.

[Nak15] _ Questions on provisional Coulomb branches of 3-dimensional $\mathcal{N}=$ 4 gauge theories, Sūrikaisekikenkyūsho Kōkyūroku (2015), no. 1977, 57-76, arXiv:1510.03908 [math-ph].

[Nak16]_ Towards a mathematical definition of Coulomb branches of 3-dimensional $\mathcal{N}=4$ gauge theories, I, Adv. Theor. Math. Phys. 20 (2016), no. 3, 595-669, arXiv:1503.03676 [math-ph].

[NY11] H. Nakajima and K. Yoshioka, Perverse coherent sheaves on blow-up. I. A quiver description, Exploring new structures and natural constructions in mathematical physics, Adv. Stud. Pure Math., vol. 61, Math. Soc. Japan, Tokyo, 2011, pp. 349-386.

[Obl04] A. Oblomkov, Double affine Hecke algebras and Calogero-Moser spaces, Represent. Theory 8 (2004), 243-266 (electronic).

[Tak15] Y. Takayama, Bow varieties and ALF spaces, Math. Proc. Cambridge Philos. Soc. 158 (2015), no. 1, 37-82.

[Tak16] _ Nahm's equations, quiver varieties and parabolic sheaves, Publ. Res. Inst. Math. Sci. 52 (2016), no. 1, 1-41. 